\title{
Analyzing Applied Calculus Student Understanding Of Definite Integrals In Real-Life Applications
}

\author{
Cody Hood \\ chood@mail.wvu.edu
}

Follow this and additional works at: https://researchrepository.wvu.edu/etd

Part of the Mathematics Commons, and the Science and Mathematics Education Commons

\section{Recommended Citation}

Hood, Cody, "Analyzing Applied Calculus Student Understanding Of Definite Integrals In Real-Life Applications" (2021). Graduate Theses, Dissertations, and Problem Reports. 8260.

https://researchrepository.wvu.edu/etd/8260

This Dissertation is protected by copyright and/or related rights. It has been brought to you by the The Research Repository @ WVU with permission from the rights-holder(s). You are free to use this Dissertation in any way that is permitted by the copyright and related rights legislation that applies to your use. For other uses you must obtain permission from the rights-holder(s) directly, unless additional rights are indicated by a Creative Commons license in the record and/ or on the work itself. This Dissertation has been accepted for inclusion in WVU Graduate Theses, Dissertations, and Problem Reports collection by an authorized administrator of The Research Repository @ WVU.

For more information, please contact researchrepository@mail.wvu.edu. 
CODY HOOD

Follow this and additional works at: https://researchrepository.wvu.edu/etd

Part of the Mathematics Commons, and the Science and Mathematics Education Commons 


\title{
ANALYZING APPLIED CALCULUS STUDENT UNDERSTANDING OF DEFINITE INTEGRALS IN \\ REAL-LIFE APPLICATIONS
}

\author{
Cody Hood
}

\author{
Dissertation submitted \\ to the Eberly College of Arts and Sciences \\ at West Virginia University
}

in partial fulfillment of the requirements for the degree of

Doctor of Philosophy in

Mathematics

Vicki Sealey, Ph.D., Chair

Harvey Diamond, Ph.D.

Erin Goodykoontz, Ed.D.

Nicole Engelke Infante, Ph.D.

Virginia Kleist, Ph.D.

Department of Mathematics

Morgantown, West Virginia

2021

Keywords: Applied Calculus, Calculus, Definite Integrals, Guided Reinvention Copyright 2021 Cody Hood 


\section{ABSTRACT \\ Analyzing Applied Calculus Student Understanding of Definite Integrals in Real-Life Applications Cody Hood}

An individual's knowledge of definite integrals can range from rote memorization to a strong foundational connection harkening back to its Riemann sum limit definition, $\int_{a}^{b} f(x) d x=\lim _{n \rightarrow \infty} \sum_{i=1}^{n} f\left(x_{i}\right) \Delta x$. In my research, I conducted seven task-based face-to-face interviews with Applied Calculus students. Through the use of real-life examples and guided reinvention, I analyzed ways in which these students, who all initially demonstrated rote memorization, could exhibit a Riemann sum based level of comprehension. This research was conducted in the confines of a student population with definite integral experience, but no formal instruction on limits. My results show that the lack of computational emphasis in class produced little to no restrictions in student development of a Riemann sum based understanding of the definite integral.

Emphasis on units facilitate this evocation of a Riemann sum definition while attempting to rationalize the use of a definite integral in an application context. The use of units also aided in the assigning of meaning to the individual symbols that construct the definite integral. By applying meaning to each symbol in the decomposition of the definite integral, students were able to better rationalize the connections the integrand, differential, and their multiplicative underpinnings have to a Riemann sum. I also demonstrate that the limit's role in student understanding of the definite integral should be dichotomized into approximation and exact sublayers. My work culminates with a sample lesson on definite integrals, which places primary focus on using population growth to elicit connections between the definite integral and its Riemann sum definition through the use of unit transformation and graphical representations. 


\section{DEDICATION}

To my daughters, Kiley and Zoey

Without them, this would have been finished much sooner 


\section{ACKNOWLEDGEMENTS}

Thank you, Dr. Vicki Sealey, for your impeccable balance of support, motivation, advice, and encouragement. Even though there were times where my progress felt like it slowed to a halt, you were always more than willing to provide feedback and were the beacon of optimism I needed to push through to the finish. You may have felt at times like you provided me nothing but criticism, but from where I'm sitting, you gave me nothing but commendation. There is no question I wouldn't have been able to complete this journey without you and for that I am forever grateful.

To the greatest person in my life, Jess, I will never be able to repay you for your patience over the past few years while I have been taking classes, conducting research, writing, writing, and writing. I am so thankful that we are still only at the beginning of our journey together. I can't wait to see what the rest of our years together have in store for us.

Mom and Dad, thank you for the lifelong support you have given me through my personal and professional life. If I ever needed an encouraging word, you all were always the first people I turned to. And Jesse, if it weren't for you, I would have never begun my academic journey at WVU. I hate to admit it, but the role model and big brother you have been for me has led me to accomplish what I have in life.

Thank you to all the members of my dissertation committee: Dr. Harvey Diamond, for your welcoming guidance as my professor and advisor during every level of my undergraduate and graduate career. Dr. Erin Goodykoontz, for never setting me up to fail and for being a friend beyond all else. Dr. Nicole Engelke Infante, for playing such a strong role in introducing me to the RUME community and teaching me so much in the early stages of my graduate work. Dr. Virginia Kleist, for being a sounding board outside the confines of my math community. The insight and perspective you brought to my committee helped shape my research to be accessible to everyone. 
Thank you to all my classmates and colleagues, Krista, Tim, Keith, Renee, Matt, and Josh, that were always willing to share ideas and resources to make this process more manageable for everyone. And a special thanks to Doug Squire, for taking me under your wing early on. Your true love for teaching has made you my biggest role model for how I want to be as an educator. 


\section{TABLE OF CONTENTS}

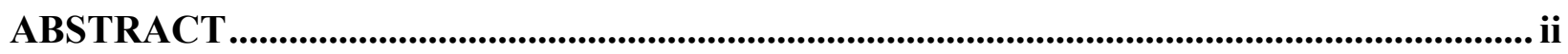

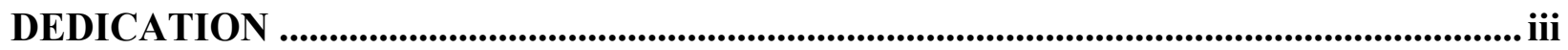

ACKNOWLEDGEMENTS ................................................................................................ iv

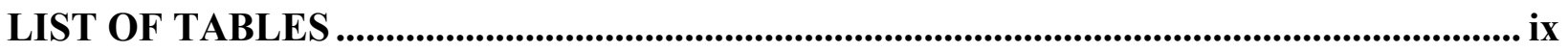

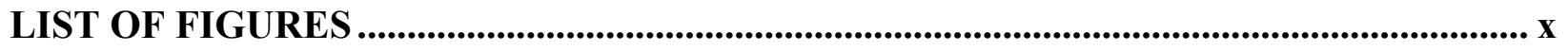

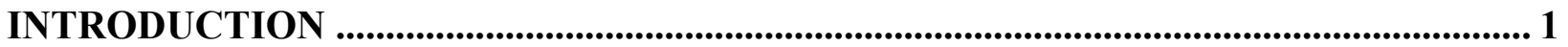

PAPER 1: STUDENT PROGRESS THROUGH THE LIMIT LAYER OF DEFINITE

INTEGRALS IN THE CONTEXT OF AREA UNDER A CURVE ..................................... 7

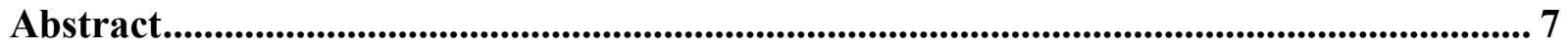

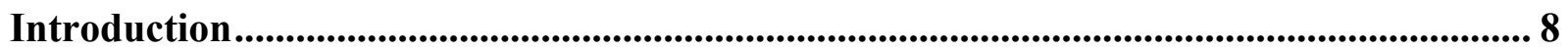

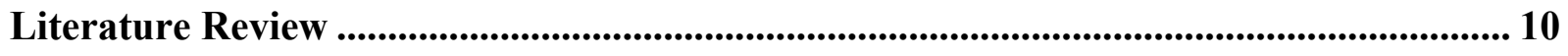

Riemann Sum Concept Image ........................................................................... 11

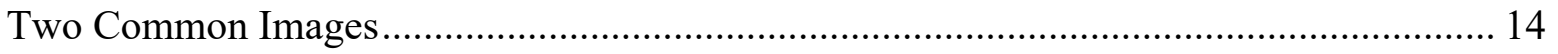

The Need for Multiple Images ........................................................................... 16

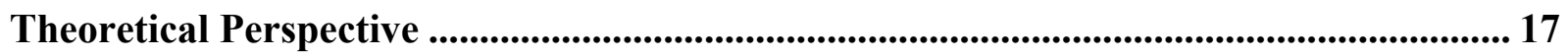

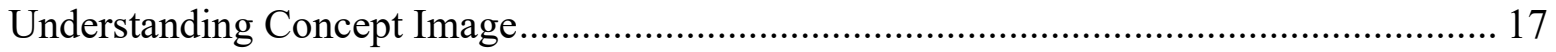

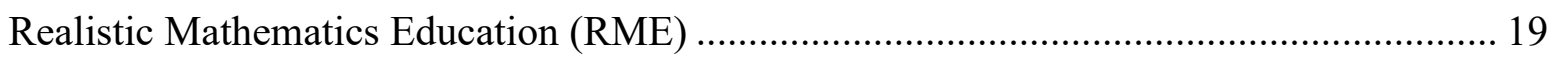

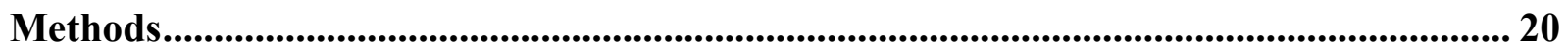

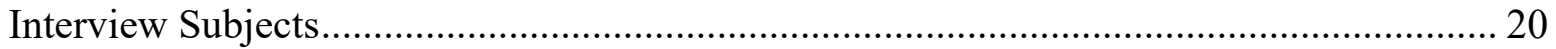

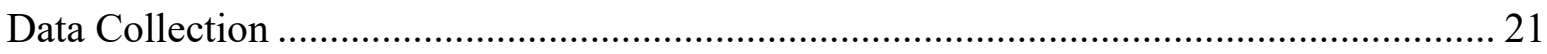

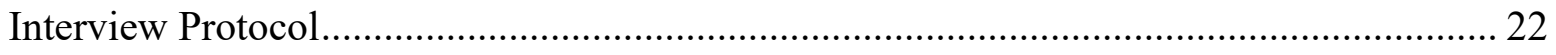

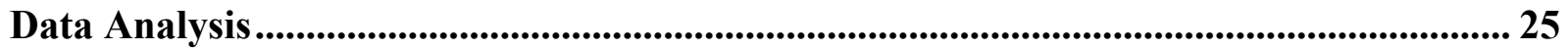

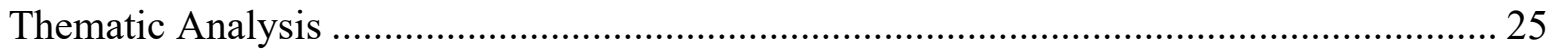

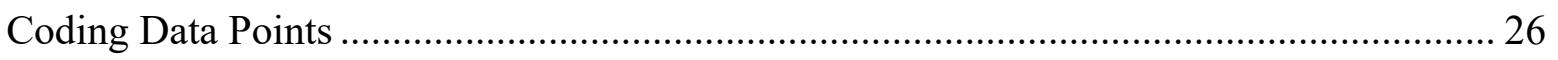

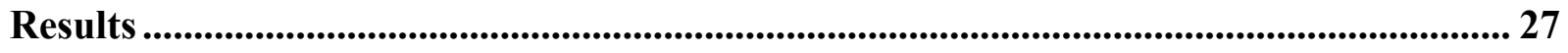

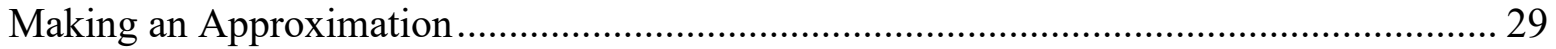




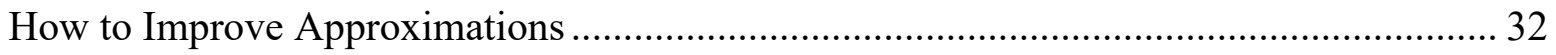

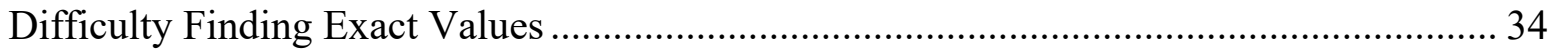

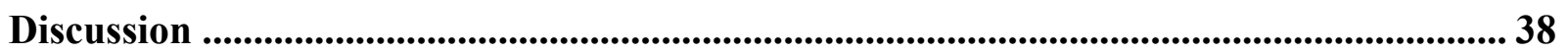

PAPER 2: DECONSTRUCTING SYMBOLIC COMPONENTS OF THE DEFINITE

INTEGRAL TO ENHANCE STUDENT UNDERSTANDING ......................................... 43

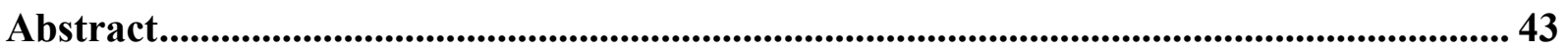

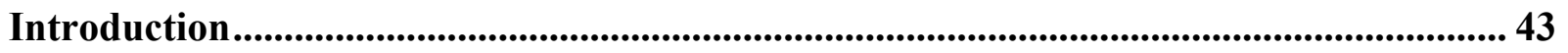

Literature Review .................................................................................................................................... 46

Riemann Sum Concept Image ......................................................................... 47

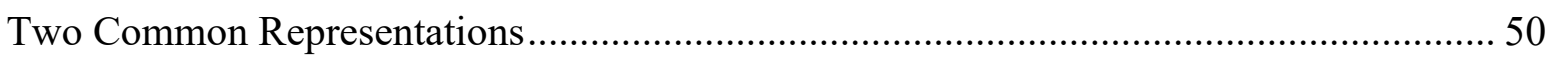

The Need for Multiple Representations ................................................................ 52

Theoretical Perspective .............................................................................................................. 52

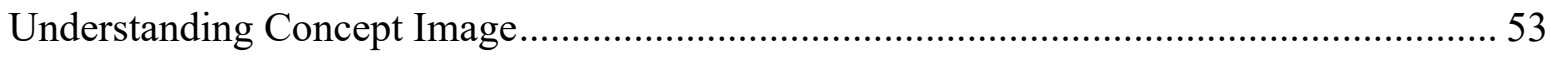

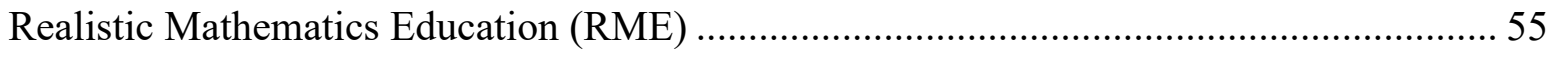

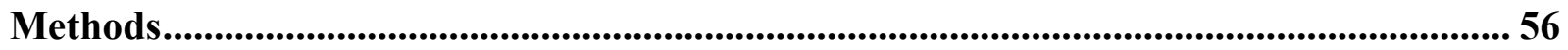

Data Collection and Interview Subjects.................................................................... 56

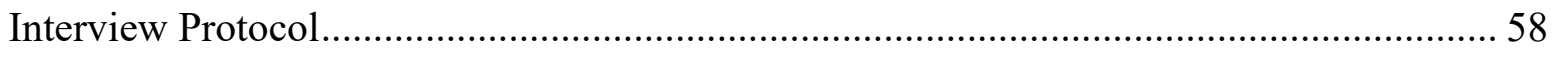

Question 1: Regarding Area Under the Curve............................................................. 59

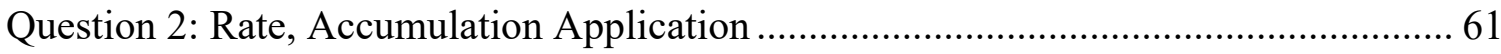

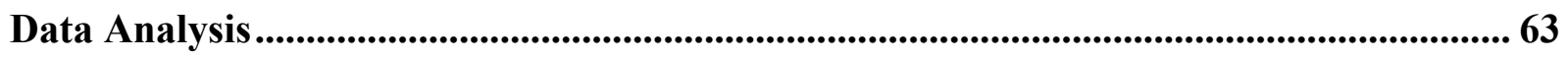

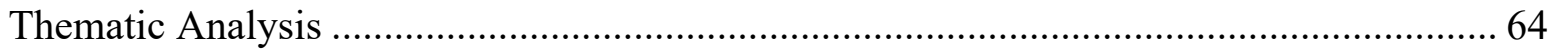

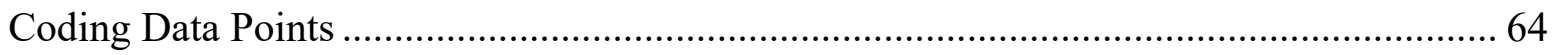

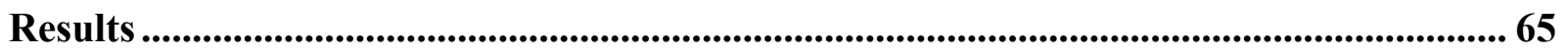

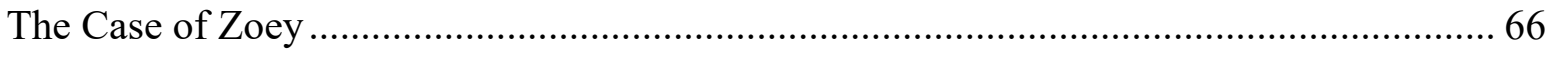

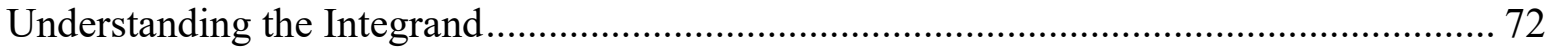

Understanding the Product of the Integrand and Differential....................................... 76

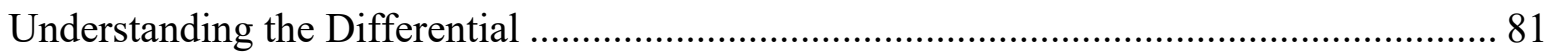

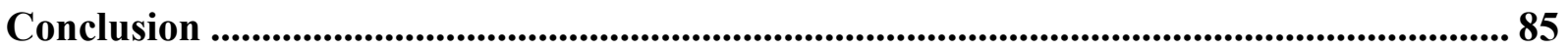




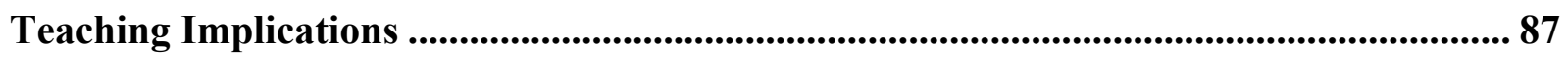

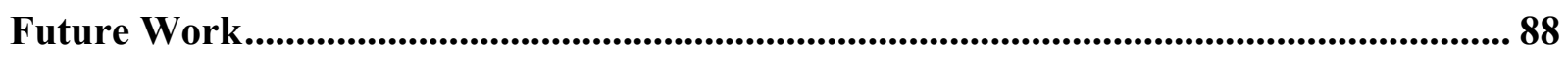

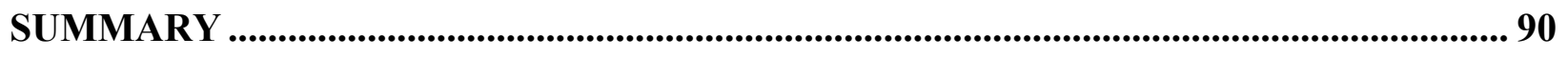

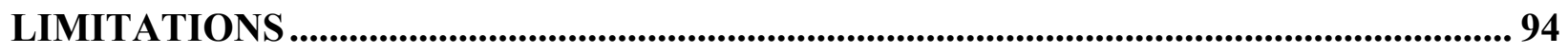

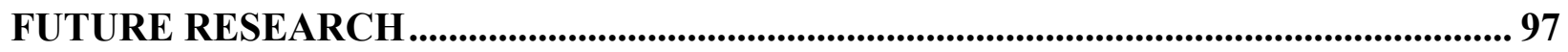

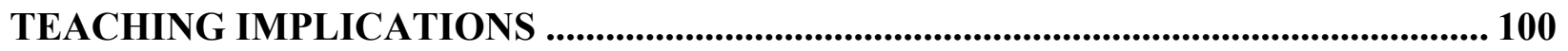

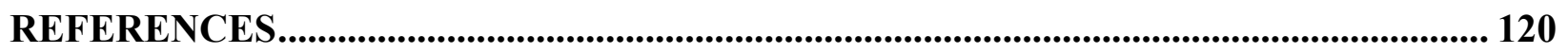

APPENDIX A: Pilot Study Interview Protocol........................................................ 123

APPENDIX B: Dissertation Study Interview Protocol ......................................................... 125

APPENDIX C: Recruitment Script ............................................................................................... 127

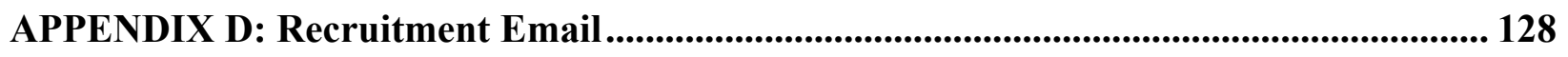

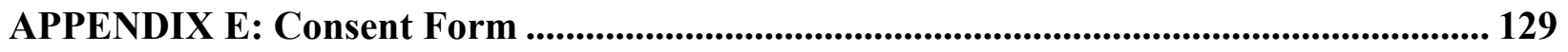




\section{LIST OF TABLES}

Table 1: Summary of Limit Layer Understanding .............................................................. 28

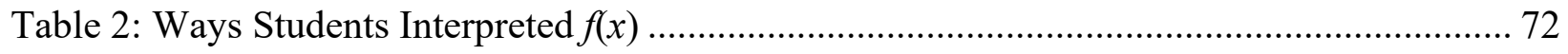

Table 3: Ways Students Wrote the Definite Integral ........................................................... 73

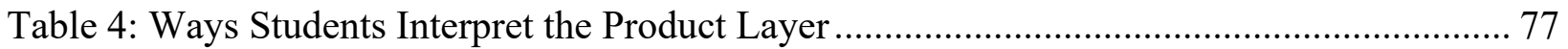

Table 5: Ways Students Interpret the Differential ............................................................... 81 


\section{LIST OF FIGURES}

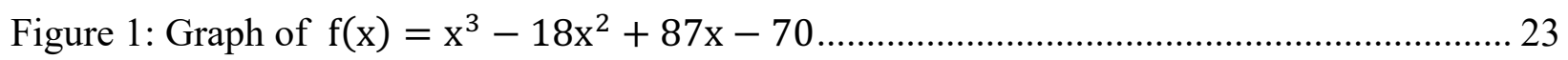

Figure 2a: Jamie initially used one rectangle................................................................ 29

Figure 2b: Multiple rectangles of equal size.......................................................................... 29

Figure 2c: Rectangles more closely aligning with a Riemann sum ...................................... 29

Figure 3a: Chase stacking rectangles vertically ........................................................... 31

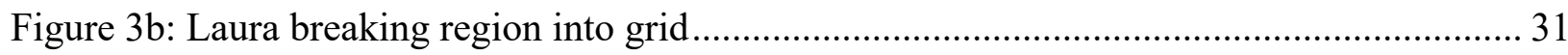

Figure 3c: Zoey using rectangles and triangles............................................................ 31

Figure 3d: Zoey identifying the cons of using only horizontally stacked rectangles ................. 31

Figure 4: Chase explaining how smaller rectangles provide less error ................................. 33

Figure 5: Laura adding up vertical lines, rather than vertical rectangles................................ 36

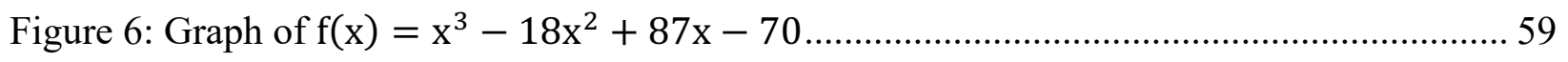

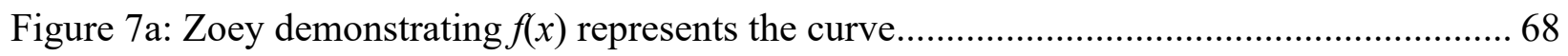

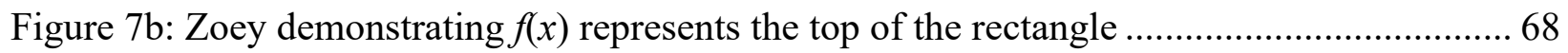

Figure 7c: Zoey demonstrating $f(x)$ represents the height of the rectangles .............................. 68

Figure 8: Different ways Zoey attributed units to the definite integral .................................... 71

Figure 9: Students demonstrating relationship between rates and amounts ............................ 79

Figure 10: Chase and Jeremy assigning units to components of the integral ........................... 80

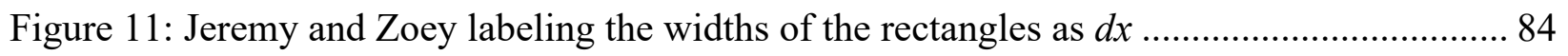




\section{INTRODUCTION}

When students express understanding of the definite integral, they typically do so in one of three ways. The two most common ways, which heavily rely on rote memorization, result from making connections to the Fundamental Theorem of Calculus or to the area under a curve (Doughty, McLoughlin, \& van Kampen, 2014; Jones, 2015; Nguyen \& Rebello, 2011, Rösken \& Rolka, 2007). When viewing the definite integral through a Fundamental Theorem of Calculus lens, students typically see the definite integral as providing instructions to determine the antiderivative, followed by arithmetic computations. The area under a curve representation can provide a prosperous visual representation of the definite integral, but typically students interpret this area as nothing more than a static depiction with little understanding of the meaning behind the construction of the definite integral. In this context, prosperous is used to describe a representation that allows for greater adaption and utilization when presented with less familiar contexts requiring a definite integral.

The least common and yet most flourishing representation of the definite integral is one that reflects its definition, harkening back to a Riemann sum. When the following connection can be made, $\lim _{n \rightarrow \infty} \sum_{i=1}^{n} f\left(x_{i}\right) \Delta x=\int_{a}^{b} f(x) d x$, there exists a high likelihood of understanding going beyond memorization for the student (Sealey, 2014). When a complete understanding of the definite integral through a Riemann sum lens exists, a student should be able to comprehend the product, summation, and limit components, often referred to as layers, that comprise a definite integral.

While no direct parallels necessarily exist, students who view the definite integral through a Riemann sum lens are afforded the opportunity to assign meaning to the symbolic components of the definite integral that help represent its origin. As an example, students with this connection 
in their knowledge base could see the multiplicative nature between $f(x)$ and $d x$ that typically is overlooked. The summation nature of the Riemann sum could potentially be encapsulated in the integral symbol, and the limit, which allows an approximation to become an exact value, has the potential to be captured in the infinitesimal nature of $d x$. By viewing a definite integral in this manner, students greatly expand upon the likelihood of understanding not only that a definite integral is necessary, but more importantly, why it is required.

In my dissertation, I explored ways students could be guided beyond memorizing the requirement for a definite integral. Unlike much of the existing research, the students I interviewed were in a calculus class that placed a heavy emphasis on real-life applications of both derivatives and integrals. This was done in lieu of definition-based and computation-based instruction. Importantly, in this class the limit was discussed as a concept but not formally written. Students were provided with anecdotal conversations about the limit and how it related to improving approximations in the contexts of both derivatives and integrals. They were never, however, asked to compute a limit nor was the limit notation ever presented to the students.

For these students, instruction on limits in the context of definite integrals never went beyond the explanation of using more and more rectangles and smaller and smaller intervals to obtain a better approximation. Class discussion also occurred around the idea that an infinite number of intervals could theoretically obtain an exact solution, but that obviously could not be computed by hand using the same Riemann sum approximation process they had previously used. Understandably, this may draw concerns, especially because recent research has demonstrated students often have difficulty encapsulating the limit layer of a Riemann sum definite integral (Jones, 2013; Meredith \& Marrongelle, 2008; Sealey, 2014). 
During clinical interviews, students were asked to explain why they knew a definite integral was needed to solve a given application problem. Students typically, and understandably, provided rationale based on the two more common definite integral representations. Not only are these quicker to recall, but compared to a Riemann sum explanation, are much more concise and easier to articulate. I began my analysis by confirming the importance of students being able to express definite integrals in these two ways. It was very beneficial for a student to almost instinctively draw connections between an area problem or a problem relating rate and accumulation to a definite integral. In this research, I took these mechanically recited explanations and asked students to draw deeper connections, in hopes of eliciting a Riemann sum understanding.

The first paper presents research on how students were able to use real-life examples involving definite integrals to rationalize the limiting nature of its Riemann sum definition. I first discovered if a method of instruction that did not involve a formal introduction to limits affected the way students were able to demonstrate understanding of the limiting process. In doing so, I showed that guided reinvention in the context of calculating an area under a curve through improved approximations could elicit from students a definite integral concept image reflective of a Riemann sum. This progress occurred in spite of the fact that all seven students interviewed initially did not provide a Riemann sum-based rationale for why a definite integral resulted in the area under a curve. I also showed that while these students demonstrated a strong proficiency in improving Riemann sum approximations in the context of an area under a curve problem, they still sometimes struggled completing the limiting process, which would result in an exact area calculation. Ultimately, I concluded that students without formal limit instruction were still able to demonstrate great progress in understanding the limit in the context of a definite integral, aided by the process of guided reinvention. The first paper answers the following research questions: 
1. Through the use of guided reinvention, how are students who do not initially approach the definite integral from a Riemann sum perspective able to adapt when required to think about the definite integral as an extension of a Riemann sum?

2. In what ways are students able to progress through the limit layer of the Riemann Integral Framework in order to bridge the potential gap between approximations of an area under a curve and the calculation of an exact value?

In the second paper, I expanded on the role guided reinvention could play in eliciting an understanding of the definite integral from a Riemann sum perspective. Again, this analysis occurred when initial student explanations were based on rote memorization. In addition to an example using area under a curve, students were provided a situation in which a definite integral was required to calculate an overall accumulation with a given rate of change. This paper analyzed the connections students were able to make between the components of a Riemann sum and the individual symbols that comprised the definite integral. These included the bounds of integration, the integral symbol, the integrand, and the differential. The purpose of this symbolic association was to aid in eliciting a Riemann sum explanation for the necessity of a definite integral in the two given contexts. Units involved in the application problem were a crucial tool for allowing students to make these connections. In this research, I was able to answer the following research questions:

1. What relationships exist between calculus students' concept images of a Riemann sum and the symbols used to construct a definite integral?

2. How can the units of a realistic definite integral application aid in guiding students through the Riemann Integral Framework?

This idea of having students develop understanding of the definite integral that relate back to its definition is not new (Jones, 2013; Jones, 2015; Meredith \& Marrongelle, 2008; Nguyen \& 
Rebello, 2011; Rösken \& Rolka, 2007; Sealey, 2014; Von Korff \& Rebello, 2012). My research expanded upon these findings. If a student provided a description resembling the two more common definite integral representations, I did not assume this is where a student's knowledge base ended. It is understandable that these representations appeared most commonly in the early stages of the interviews conducted for this research because of their ease of accessibility. I used these less prosperous representations, partnered with real-life applications, to help elicit deeper understanding that a student may possess, but did not necessarily express naturally.

This research also heavily utilized the symbolic construction of the definite integral as a tool to stimulate responses more reflective of the definition of the definite integral. As stated earlier, while the individual components of the definite integral may not intrinsically have meaning, a student can anecdotally apply meaning to the integrand, differential, and their interaction in order to draw strong connections that relate back to the definite integral's definition.

My research suggests that while struggles understanding the limit in the context of the definite integral occasionally existed, they were in no way exacerbated by the means of instruction, which did not include formal instruction on limits. In actuality, as this research shows, many of the students in these interviews demonstrated very prosperous and even complete understanding of the limit concept and were able to justify its significance in an integral. It is important to remember that these accomplishments occurred without any formal instruction on limits in class. These results provide evidence that instruction including real-life, conceptual meaning of limits and, in turn, definite integrals has a positive influence on a student's ability to express understanding of a definite integral reflective of its Riemann sum definition.

From these results I have also developed ways in which student learning and understanding of definite integrals can be enhanced in the classroom setting. I discussed how my research could 
affect teaching of definite integrals holistically and explained how real-life examples and guided reinvention could play a vital role in bridging the gap between the student and the abstract nature of definite integrals. I followed this philosophical overview of instruction with an overall reminder to educators that what a student demonstrates in the context of a given application is not always reflective of his or her knowledge. It is the facilitator's responsibility to develop tools and techniques to elicit the desired representation of knowledge by a student. Only by having the proper tools in place can a student's level of understanding accurately be monitored and expanded.

An example of a lesson used to elicit a Riemann sum concept image of the definite integral is also presented. I outlined how instruction on definite integrals could be presented to encourage students to avoid reliance on rote memorization tools and shift to a deeper understanding of a definite integral's definition. I ended this outline with an activity demonstrating how a graphical representation of population growth could be used to evoke connections between definite integrals, graphical representations, unit transformations, and most importantly, a definite integral's Riemann sum definition. 


\title{
PAPER 1: STUDENT PROGRESS THROUGH THE LIMIT LAYER OF DEFINITE INTEGRALS IN THE CONTEXT OF AREA UNDER A CURVE
}

\begin{abstract}
Amongst experts, there is a consensus that the most prosperous way for a student to perceive the definite integral is through a Riemann sum lens, where students view an integral as a sum of multiplicative pieces. Our research presents a subset of data from a larger study on how best to elicit student understanding of the definite integral. In this paper, we present an analysis of how Applied Calculus students were able to use guided reinvention to successfully progress through graphical representations of a Riemann sum. This Applied Calculus course differed from many other stem-based calculus courses in its heavier emphasis on applications, with no formal instruction of the limit being provided. This course was afforded the ability to discuss the concept of a limit only conceptually because it was considered a terminal course for the students enrolled. This course was not able to be used as a prerequisite to progress through the calculus sequence, which allowed for increased emphasis to be placed on conceptual and application-based understanding of calculus concepts. Even so, we will show these students possessed a substantiated ability to encapsulate much of the limiting nature of a Riemann sum and a definite integral. In order to obtain a fuller understanding of the definite integral, a student may decompose the definite integral into product, summation, and limiting components.

This paper explores how students were able to maneuver through the limiting layer as a way of improving their conceptualization of the Riemann sum. In particular, we focused on a collection of students who had not had formal instruction on limits. Here, we describe what concepts students struggled with and what aspects of their understanding were novel and less predictable to the researchers. By utilizing the visual aids that area under the curve representations
\end{abstract}


of the definite integral provide, we determined that students were able to construct approximations, improve upon these approximations, and even rationalize how to determine the exact area under the curve through the use of rectangles and various other geometric shapes. Ultimately, we claim that student understanding of the limit in a definite integral should be divided into two sublayers, one related to improving approximations and the other focused on obtaining an exact value. This work contributes to the stream of scholarship that yields improved methods in teaching calculus.

\section{Introduction}

In the calculus sequence, definite integrals play a very important role in a variety of applications. Frequently, heavy emphasis is placed on computationally calculating an integral, rather than obtaining a foundational understanding of what the integral represents in a mathematical sense. This can lead to a lack of understanding of the definite integral from the perspective of a student truly aligning with its definition. When deciding if a definite integral is required in a certain application problem, students often call upon three potential methods of justification from their own concept image. Two of the typically used student methods are rooted in the use of recall. The first of these methods involves the student developing a reliance on reducing a definite integral to nothing more than the area under a curve (AUC). Research has shown that an AUC understanding alone is not prosperous to yielding a rich ownership of the integral; it is only when students can unpack the connection between AUC and a definite integral does the AUC approach truly become useful (Sealey, 2006). A student relying on previous examples, rote memorization and the Fundamental Theorem of Calculus (FTC) is the second method of recall commonly used. Students that show a reliance on the FTC view the definite integral as a command to discover the integrand's antiderivative, plug in the bounds of integration, and then calculate the difference of these two values. Again, this second approach is less than fully 
prosperous in allowing the student to develop a robust understanding of the problem that is being addressed.

Most prosperous however, is a rationalization built upon an infinite summation of products over a finite interval (Jones, 2013; Jones, 2015; Meredith \& Marrongelle, 2008; Nguyen \& Rebello, 2011; Rösken \& Rolka, 2007; Sealey, 2014; Von Korff \& Rebello, 2012). In this paper, this is referred to as a Riemann sum concept image. There are many layers of understanding required to fully internalize this level of understanding when justifying the use of a definite integral via a Riemann sum. Beginning with an approximation, we say that a student must identify a sequence of products $\left(f\left(x_{i}\right) \cdot \Delta x\right)$ being summed, represented by $\sum_{i=1}^{n} f\left(x_{i}\right) \Delta x$. This approximation is improved upon by making $n$ large. A limit can even be applied to make $n$ as large of a finite number as one would like, $\lim _{n \rightarrow N} \sum_{i=1}^{n} f\left(x_{i}\right) \Delta x$ for large $N$. For full comprehension, the final characteristic of this limit layer that must be understood to obtain an exact value is replacing large $N$ with infinity, $\lim _{n \rightarrow \infty} \sum_{i=1}^{n} f\left(x_{i}\right) \Delta x$. Only when the value of $n$ tends towards infinity and the interval size, $\Delta x$, becomes infinitesimal, can it be said that a student fully incorporates the limit layer of the Riemann Integral Framework into his or her concept image of the definite integral.

In this study, we observed how Applied Calculus students' progression through the Riemann Integral Framework (Sealey, 2014) occurred via the aid of visual tools in the context of calculating an area under a curve. While this selection of students was introduced to the idea and concept of limits, they were provided no formal instruction of limits. At no point in their Applied Calculus course were they asked to compute or even write a limit. Despite these differences in instruction, we sought to understand how well these students would be able to apply the notion of limits in order to improve approximations and ultimately obtain the exact value a definite integral represents. This was accomplished by having students draw connections between an area under a 
curve calculation problem, how it could be approximated, and why the definite integral provided this resulting calculation.

A sample of students, provided with an arbitrary graph, were tasked to develop a Riemann sum definition for the definite integral through the use of graphical approximations of the area under the given curve. Students began by developing an approximation of the area using geometric tools and then progressed towards an exact value. By examining the problem using a process of iterating improvements on a graph, we determined that students were able to physically see what their approximations were calculating and how they could be improved. This approach provided the students with additional insight into how to incorporate the concept of the limit into their understanding, even without it being formally taught in class. In future work, we will discuss how this progression provided insight into a student's ability to deconstruct and provide meaning to the symbolic components of the definite integral. Through this paper, we address and answer the following research questions.

1. Through the use of guided reinvention, how are students who do not initially approach the definite integral from a Riemann sum perspective able to adapt when required to think about the definite integral as an extension of a Riemann sum?

2. In what ways are students able to progress through the limit layer of the Riemann Integral Framework in order to bridge the potential gap between approximations of an area under a curve and the calculation of an exact value?

\section{Literature Review}

In order for students to be successful in properly applying definite integrals to many nontrivial engineering and physics applications, rote memorization of when a definite integral is required will not necessarily lead to student success. Especially if a scenario is new or not typically 
utilized, understanding the definite integral in the context of its Riemann sum foundation is all but required (Jones, 2013; Meredith \& Marrongelle, 2008; Orton, 1983; Sealey, 2014; Thompson, 1994). In order to fully explain how students understand the definite integral, it is important to first discuss common images students possess of the definite integral, common misconceptions and pitfalls of each image, and which image best aligns with understanding applications involving the integral. The last of these requirements can be arguably the most important characteristic of a productive image of definite integrals. While each image of the definite integral can contain what Orton (1983) describes as arbitrary errors, such as arithmetic mistakes and improper execution of evaluating a definite integral, we believe a bigger focus should be placed on misconceptions that are derived from structural errors. These errors stem from a lack of understanding of the intricacies of the definite integral and result in "holes" and "gaps" in an individual's image of this complex concept.

\section{Riemann Sum Concept Image}

Regarding many core concepts within mathematics, including functions, limits, and integrals, there is a strong collection of experts who believe it is important to provide instruction that allows for the creation of an image resembling the formal mathematical definition of the particular concept (Jones, 2013; Jones, 2015; Meredith \& Marrongelle, 2008; Nguyen \& Rebello, 2011; Oehrtman, 2009; Rösken \& Rolka, 2007; Sealey, 2014; Von Korff \& Rebello, 2012). Unfortunately, many students fail to ever reach this level of understanding of the definite integral (Doughty, McLoughlin, \& van Kampen, 2014). This is not saying that a mathematical definition should be identical to the image being evoked of a particular concept, nor are students at their respective educational levels mathematically mature enough to fully comprehend the rigorous mathematics and notation associated with a given definition. The argument simply suggests that if 
a student is introduced to a particular concept in a manner that resembles the way the certain concept is mathematically defined, then that student will have a stronger understanding of what that concept actually is. In turn, the student will have the building blocks to strengthen this concept image at a later point, more closely aligning it to the formal definition of the concept.

Concerning the definite integral, there are multiple names referring to the concept image that reflects how students come to understand the formal definition of the definite integral. These terms include parts-of-a-whole, accumulation of rates, adding up pieces, and multiplicativelybased summations (Jones, 2015; Rösken \& Rolka, 2007; Sealey \& Thompson, 2016). Because all these potential concept images involve some variation of understanding the definite integral when viewed through a Riemann sum lens, all these titles will be consolidated into the Riemann sum concept image of the definite integral for the remainder of this paper. Attempting to create an image that closely resembles the definition of the definite integral demonstrates a very robust understanding. In contrast to concept images based in memorizing when its use is required, the Riemann sum image of the definite integral will not only guide a student to better understand from where the definite integral is derived, but also teach to its importance, when it is required to be used, and how to use it properly (Sealey, 2014).

We will be using Sealey’s (2014) Riemann Integral Framework (RIF) to describe the way students could potentially be viewing the definite integral through a Riemann sum concept image. As a summary, Sealey's (2014) framework breaks student understanding of the definite integral into four layers and one pre-layer. The pre-layer, orienting, "includes visualizing the situation, understanding the variables in the problem, and understanding the quantities in the problem" (p. 237). Following the pre-layer, the product layer requires students to conceptualize the quantity formed by the product of $f(x)$ and $\Delta x$. The second layer of visualization is that a student should 
also be able to conceptualize the summation layer, which involves understanding that the summation of these products results in an approximation of the definite integral. In order to turn this summation approximation of the definite integral into an exact value a student must possess the ability to apply a limit to this summation, resulting in infinitesimally small $\Delta x$ intervals, leading to the concept of $d x$, which constitutes the third layer of the RIF, the limit layer. The final layer of the Sealey RIF, function, requires a student to be able to view this definite integral as a function rather than a static entity. Taken together, the pre-layer and subsequent additional four layers combine to yield a substantive understanding of the definite integral (Sealey, 2014). Sealey also noted that it was not necessary or even expected for a student to progress through understanding the layers in order, and that often students will jump back and forth between layers when working to solve a problem.

Sealey's (2014) work shows that the difficulty of reaching a final exact value may also be a result of failing to make the connection between Riemann sum approximations and the FTC to actually compute an exact value. Sealey's students seemed comfortable in understanding that an exact value exists, but, nevertheless, could not connect the continually improving approximations to the definite integral. Many students possess the ability to understand, through their own image of the Riemann sum, that smaller intervals of approximation, and in turn more intervals, will result in a better approximation of the definite integral. What students fail to develop is the ability to extend this idea infinitely using the limit to achieve an exact value (Jones, 2013; Sealey, 2014). Meredith and Marrongelle (2008) state that, 'students' previous experiences with the word 'limit' contribute to the way in which they make sense of the concept of limit" (p. 570). If the limit concept image is not developed correctly or cannot be extended to situations outside of derivatives and algebraic calculations, it is unlikely that a student will possess the ability to move toward the 
rationale that smaller rectangles will result in a better approximation leading to an exact value (Cornu, 1991).

It has been observed that students frequently lack a structural understanding of the purpose of the limit and as a result, are unable to apply it properly to the Riemann sum (Orton, 1983; Sealey, 2014). Even when an individual has a strong understanding of the limit, he or she may not understand the necessity of it in a particular situation. Many students fail to perceive functions from a dynamic perspective that represents the relationship between two or more quantities, but rather as a single, static entity (Sfard, 1991; Sfard, 1992). The same can be said of the Riemann sum (Jones, 2013). When students perceive a Riemann sum, it is often understood as an approximation that is dependent upon a given, fixed interval size. These intervals, or "chunks" as Thompson (1994) describes them, are fixed for each representation of the Riemann sum approximation. Many students lack the ability to see a dynamic relationship between the Riemann sum approximation, its corresponding interval size, and how the accuracy of this Riemann sum is a direct reflection of this relationship (Sealey, 2014). If students are able to view the Riemann sum as a dynamic process, with $\Delta x$ not having a fixed size, the idea of incorporating the limit will likely become much more intuitive. This dynamic representation of the Riemann sum can aid in pushing beyond approximations, resulting in an exact value.

\section{Two Common Images}

One of the most common types of images formed of the definite integral is comprised simply of rote memorization of the previously discussed Fundamental Theorem of Calculus (FTC). This rote memorization occurs when a student views a definite integral as a command to find a function's antiderivative (Hall, 2010; Jones, 2015). A second common concept image of the definite integral is a static visual representation of Area Under the Curve (AUC) (Doughty et al., 
2014; Jones, 2015; Nguyen \& Rebello, 2011, Rösken \& Rolka, 2007). We note that it is certainly possible, and even desirable, for students to unpack area under a curve in a way that views the total area as a sum of the areas of infinitely many rectangles. In this paper, we would classify this as a Riemann sum perspective and reserve the AUC perspective as only viewing the area as a static single entity. In Hall's (2010) study just over two-thirds of the students viewed the definite integral as a command to perform a computation involving simple recall cues or as a static area under a curve, rather than as a Riemann sum or other various representations. Both weaker representations, which constitute the two common images of the definite integral described in this section, demonstrate that many students rely on memorization and static visual representations rather than connecting back to rigorous definitions.

By having a narrow image of the definite integral that is reflective of only recalling previous examples, there is little meaning that can be derived and utilized in a real-life application (Nguyen \& Rebello, 2011). Even still, it is arguable that in calculus courses, the greatest emphasis in definite integrals is placed upon a student's ability to evaluate a definite integral. According to Bressoud, Mesa, and Rasmussen's (2015) nationwide study, 50\% of the problems given to students in Calculus 1 are strictly skill based and computational. In comparison, only $10 \%$ of problems involve less familiar application situations. When the focus of the definite integral is solely its evaluation, the meaning and purpose of each aspect of the definite integral and its notation is severely limited or even obsolete (Doughty et al., 2014; Thompson, 1994).

According to Jones (2013), an AUC concept image is oftentimes the second most common mental representation of the definite integral, behind the FTC concept image. The area under the curve's ability to provide students with an easy-to-understand static visual representation of the definite integral leads to it having such a strong presence in many concept images. We believe this 
image of the definite integral is often called upon by students because of its ability to bring some physical meaning to a numeric and abstract process. Unfortunately, just like the FTC representation, the AUC concept image is an oversimplification that can create many misconceptions. Understanding the definite integral as area under the curve can help students understand simplistic applications such as rates of change in distance with respect to time, but this approach can fall short in aiding the understanding of applications that cannot be represented twodimensionally (Nguyen \& Rebello, 2011; Sealey, 2014; Thompson, 1994). While this image does have the ability to incorporate notational aspects of the definite integral into its concept image, it is often done incorrectly and carries limitations as well.

\section{The Need for Multiple Images}

While there are many arguments concerning why different images of the definite integral are constructive to a student's understanding, all provide valuable information concerning meaning. Each image of the definite integral that has the potential to be evoked also may contribute to misconceptions and incorrect assumptions. Fortunately, "a student's concept of integration may not be a single entity, but rather be made up of smaller units, including ideas of area, antiderivatives, summations or differentials" (Jones, 2013, p. 123). This incorporation of different images into a single concept image of the definite integral provides students with an ability to view definite integrals from multiple perspectives, preventing misconceptions from occurring (Doughty et al., 2014). It should be noted that just because many students possess multiple images of the definite integral simultaneously, it cannot be assumed that when a particular image is not evoked, the student does not possess that particular image (Jones, 2013). If a student is unable to correctly solve a problem that incorporates the definite integral, it also may not be directly assumed that the inability results from a misunderstanding of the definite integral. In short, students may understand 
the definite integral through numerous images, but may not be able to synthesize the images into a cohesive whole to achieve the appropriate resolution.

\section{Theoretical Perspective}

The leading theoretical perspective that drove the research described in this paper was the seminal work on concept image by Tall and Vinner (1981). The major objective of our research questions was to advance literature by analyzing the definite integral concept images found with non-STEM students in an Applied Calculus course. An individual's concept image, which can be described as the collection of knowledge a person associates with a particular concept, is not a static entity. It is constantly expanding, developing, and being modified as a person encounters new situations (Meel, 2003). Thus, the approach to our research was to explore the dynamic nature

of the concept image with a specialized student population. Additionally, the framework of Gravemeijer and Doorman's (1999), Realistic Mathematics Education (RME) was used to guide the interview protocol.

\section{Understanding Concept Image}

Theoretically, complete understanding of a concept can be obtained directly from the application of its formal definition. Realistically, however, this is never actually the case. In comparison to a formal concept definition, which is universally accepted, each individual creates a personal concept definition, which defines the given concept based upon an individual's understanding, whether correct or not (Davis \& Vinner, 1986; Meel, 2003; Tall \& Vinner, 1981). Through interacting with a concept, students continually add, remove, and modify information that they associate with a particular concept as they become more familiar with it to answer questions and draw conclusions. In fact, in many undergraduate courses, students can associate ideas with a concept without even knowing or understanding what its formal definition is. This collection of 
ALL structures a student associates with a concept is called the concept image (Tall \& Vinner, 1981). Importantly, a student's concept image, which is unique to that individual, contains more than just an informal definition.

Amongst other subsets, the concept image can contain symbols, ideas, formal definitions, personal definitions, visual representations, and varying examples that an individual associates with a given concept. As time progresses, improvements can be made to a concept image. This can only occur when conflict arises. The idea that potential conflict always exists but has yet to be evoked is called the potential conflict factor (Tall \& Vinner, 1981). These factors are inconsistencies that are prevalent in a person's concept image but have yet to be brought to the individual's attention. Once these conflicts are brought to light and encountered, they become cognitive conflict factors for an individual as they work to solve problems. This means that the person whose concept image contains these conflicts becomes aware of the inconsistencies and is faced with the task of modifying the current concept image to alleviate this conflict.

Determined by the given context of the current situation, only a portion of a student's concept image is ever elicited at a given time (Doughty et al., 2014). This idea can be referred to as an evoked concept image (Tall \& Vinner, 1981). The subset of a person's concept image can change based on an enumerable number of factors (Meel, 2003). One must be careful not to assume that a person's evoked concept image is a valid representation of his or her complete concept image. This distinction is currently missing from current research on student understanding of the definite integral. A major goal of our research is to explore this distinction between evoked concept images and evocable concept image. It is important for someone to be able to successfully elicit the appropriate tools to understand the necessity of a definite integral. By identifying these 
conflicts, not only is a student able to improve upon his or her current concept image, but it also distinguishes between the ability to possess knowledge and the ability to evoke it.

\section{Realistic Mathematics Education (RME)}

While concept image was the primary framework used to guide our research, as indicated earlier, RME was used as well. Gravemeijer's (1999) RME was used as a tool to help guide students in an attempt to better understand aspects of their concept image regarding definite integrals. Much of what we studied involved how students were able to learn through guided reinvention. Through the use of RME, a goal of ours was to better understand students' ability to comprehend the definite integral through a Riemann sum concept image. Even if students approached problems initially with an AUC or FTC concept image, we searched to determine how understanding could be further constructed to elicit deeper meaning.

Gravemeijer and Doorman (1999), researchers at the forefront of RME, believe that with the aid of the environment, students are able, "to (re)invent the more formal mathematics" (p.159). The use of the word "(re)invent" attempts to illustrate the balance between discovery and creation of truth. This use of guided reconstruction allows for students to make connections between mathematics and their own reality that otherwise would be severely limited or possibly insurmountable (Gravemeijer, 1999; Gravemeijer \& Doorman, 1999; Hough \& Gough, 2007; Treffers, 1993). A metaphor Prawat and Floden (1994) have for RME expresses the idea that truth unfolds, given a context. Truth is not something eternal, but rather emerges when deemed necessary (Gravemeijer, 1999; Prawat \& Floden, 1994). The RME is rooted in the belief that truth emerges from both individual creation and environmental discovery. 


\section{Methods}

\section{Interview Subjects}

The students we interviewed were concurrently enrolled in an Applied Calculus course at a large public university in the United States. These students volunteered from the six in person sections of 80-100 students that were offered that semester. While the lectures from class sections were not recorded, one of the authors was the course coordinator for this highly coordinated class. The students in this class were introduced to the necessity of the integral in basic applications, how to computationally calculate the definite integral, and more advanced applications of the integral. These more advanced topics included calculating future and present value given an income stream, as well as consumer and producer surplus. During the initial introduction to basic applications, students were presented with a table and graph of velocities at given times and were tasked with calculating distance traveled over a given amount of time. This was done in a manner that mimics the Riemann sum concept image of the definite integral with one glaring omission. While the idea of the limit of the Riemann sum was introduced, this method of instruction omitted explicit use of the limit notation. For these students, the limit was perceived more as a concept rather than something that can be mathematically calculated. One driving purpose for this exclusion is the course's enhanced focus on conceptual understanding through guided reconstruction as opposed to rigorous proof and computation.

Students in this course had also seen in class what a Riemann sum approximation looked like graphically, as a summation of rectangles. It was discussed why rectangles, as opposed to other shapes were used for approximating, and how these approximations could be improved upon by reducing the widths of these rectangles. The culmination of the graphical aspect of this unit was understanding that a Riemann sum could approximate the area under a curve and the definite 
integral computes this exactly. Heavy emphasis in this course was placed on understanding why any integral is necessary in applications, so once demonstrating an $80 \%$ level of mastery in calculating antiderivatives, students were permitted to use calculators to compute definite integrals for the remainder of the semester.

\section{Data Collection}

Upon completing the majority of the definite integral unit during their Applied Calculus class, students were asked by their instructors to participate in our study involving material they had learned in the course. Each instructor was provided with a recruitment script to be read during class. This script included generic information about the study and provided a sheet for students to use to sign up. An additional email was sent out to recruit students as well. At no point in time prior to the interviews were students informed that the interview would be related to definite integrals. The students received no compensation, monetary or otherwise, for their participation.

Students who had elected to participate were reminded via email that interviews would be videorecorded and were asked to sign up for an interview time. Subjects who attended their interview time were given the consent form and an opportunity to ask any questions they may have had prior to beginning the interview. Videorecording began once subjects signed the consent form. Pseudonyms were used in place of real names to keep student information confidential.

During our study, a semester in which one of the authors was a course instructor, we conducted seven interviews. In these interviews, there was also a third person in the room videorecording the interview. Originally during the pilot study, we had anticipated that students would be able to work together in pairs and build from each other's ideas to help develop and discover a deeper understanding of the definite integral. Unfortunately, the interview subjects often showed reservation in expressing personal mathematical beliefs. Despite multiple attempts by the 
researchers, ideas were rarely shared with each other or with the interviewer. Our hope was that by not having students interview in pairs, we would be able to get a more accurate depiction of how each individual perceived the definite integral. We did not want the students we were interviewing to withhold any thoughts or ideas because they were afraid they would be wrong. By not having other students in the interview, we were able to discourage this fear.

These interviews ranged from 40 to 50 minutes in length. In addition to some handouts we provided, the students were also given a whiteboard to write down any thoughts they may have had. We continually tried to encourage the interviewees to write down as much as they could in regard to what they were thinking in order to capture as much of their evoked concept image as possible.

\section{Interview Protocol}

While the interview protocol was designed to be strictly adhered to, exceptions were made. As a result of time constraints, certain questions or parts of questions were omitted from some interviews. The interview protocol was also designed in such a way that it allowed for flexibility based on student responses. This flexibility also allowed for a more natural guided reinvention of the definite integral through the use of two real-life examples to occur. Follow-up questions were often asked when additional clarification was needed, when a response was found particularly interesting, or when a comment contradicted a previous statement by the student.

We began each interview by providing the following graph in two formats (Figure 1). To the best of our ability, for each of the students in our sample, we drew this graph on approximately one-third of the whiteboard to encourage student interaction. We also provided students with a small printout version of the graph to ensure no details were lost through hand-drawing. 


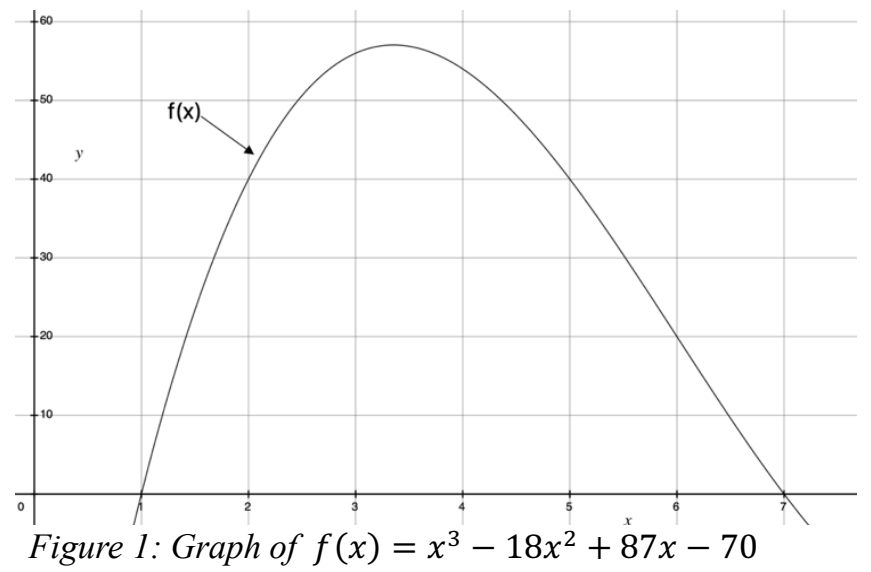

The function we chose to use was $f(x)=x^{3}-18 x^{2}+87 x-70$. Since one of our research goals involved students using Riemann sum rectangles to approximate the area under the curve, we wanted to use a function that allowed for the construction of multiple rectangles with a net positive area. If a student decided to use rectangles with a width of one for this function, five rectangles with a positive area would exist, aligning with a standard Riemann sum mental picture. Even if a student decided to use rectangles of width two, there would still be multiple rectangles with a positive area in the drawing.

Once the students were provided with this graph, we asked them, "How would you go about finding the exact area of the following region above the $x$-axis?" Even though this was the first question being asked in the interview, much of the rest of the interview attempted to draw some sort of comparisons between the approximation of the area, the Riemann sum, the symbolic components of the definite integral, and the exactness of the definite integral. Because there was frequent transition between exact calculations and approximations during most of the interviews, we believed it was very important for the instructions to clearly distinguish between the use of an approximation and an exact value.

After the definite integral was set up by the student, but before asking to actually calculate the exact area under the given curve, we asked for an estimation of this area. The actual calculation 
was not a main point of interest to us. Instead, this addition to the protocol helped us better understand what method a student would use to find an approximation. In doing so, interviewees were encouraged to geometrically approximate the area. Once the students provided us with a method for approximation, we followed-up by asking how he or she could make this approximation better. A primary outcome we attempted to achieve from this question was the connection between individual symbols that comprise the definite integral, the Riemann sum, and area under the curve. We also sought to understand to what degree students were able to reconstruct a Riemann sum understanding of the area under a curve. It is important to reiterate that one of the major goals of our study was to determine what concept images students have the ability to possess/develop, not just which ones were naturally evoked like much of the existing research has done thus far. We were not interested in discerning between newly constructed understanding and elicited understanding. We were interested in what understanding could be present by the end of the interview that was not initially evoked.

Students were then asked to improve upon the approximation they provided. While freedom was given to the students in determining how this improvement would occur, we did guide each student by reforming their first approximation to include only vertical rectangles if not done so naturally. This improvement in approximation was expanded upon once more to see if students possessed the ability to fully encapsulate the limit layer of the RIF by theorizing how an exact solution would be obtained. Our goal was to better understand to what degree these students progressed through the varying layers of a Riemann sum and how understanding could be developed by expanding upon a graphical approximation to eventually obtain an exact answer.

While the overall goal of these interviews was to see how well Applied Calculus students could progress through definite integral problems, one of the most interesting aspects that emerged 
from the data was based on students' understanding of the limit layer in Sealey's (2014) Riemann Integral Framework. We answered our research questions by asking students not only how they could improve upon their approximations but also why they knew an improvement occurred. Ultimately, we hoped for students to progress through the limit layer by developing an approximation for the area under a curve, improving this approximation, and eventually connecting to the definite integral and an exact value.

\section{Data Analysis}

The primary purpose of our analysis was to understand how students could present an understanding of the definite integral from a Riemann sum perspective regardless of whether a Riemann concept image was initially evoked by the student or was a result of guided reinvention via the interview. Particular attention was given to the limit layer. A secondary goal of our analysis was to determine to what extent current literature on student understanding of the definite integral aligned with the population group of Applied Calculus students with whom we worked. Again, it is important to remember that this collection of Applied Calculus students had not seen the formal use of limits at any point in the course.

\section{Thematic Analysis}

We began data analysis in a manner following Braun and Clarke's (2006) thematic analysis for qualitative analysis to answer our research questions. Below, we will be describing the process we used to familiarize ourselves with the data collected in the form of video recordings and handwritten notes. We will also be describing the methods used to generate, identify, define, and connect themes within and across interviews. As an aid to conducting thematic analysis, Strauss and Corbin's (1998) method of axial coding was also used to decompose the themes we discovered. 
This was the primary technique we utilized to help identify and confirm themes that existed across research participants.

There are two primary ways in which thematic analysis can be conducted, inductively and theoretically. Inductive thematic analysis occurs when themes are drawn completely from within the data, with minimal previous bias. When theoretical analysis is used, the researchers' bias comes into play in predicting which themes may be developed. The research then tests these hypothesized themes to determine their validity. When an inductive approach is used, it provides a richer description of the data because no preexisting influence is taken into account. When a theoretical approach is used, a more detailed description of the themes can develop because they have been predefined prior to analysis (Braun \& Clarke, 2006).

While this research does build upon preexisting research, an inductive approach was used to develop the themes that will later be discussed. The questions asked in the interview protocol were not designed to draw out specific themes but were instead left to elicit more open-ended discussion by the students. This method of analysis was important to ensure data was not being used to force replication of preexisting research on how students understand the definite integral through a Riemann sum lens. From the data, themes were extracted, rather than using the same data to support hypothesized themes. As we will see, themes quickly developed that helped to provide insight into the ways students progress through the limit layer of a definite integral. This included developing approximations, improving these approximations, and incorporating infinity into the limit in order to obtain an exact solution.

\section{Coding Data Points}

During the initial stages of analysis, we watched each interview recording at least three times. During the first pass at the interviews, we time stamped any information we considered 
potentially relevant to our research questions. This included information that related to the phases of Sealey's (2014) RIF, the three common concept images of the definite integral, and any information regarding student understanding of the symbolic construction of the definite integral. Once this first pass at the data was complete, we returned to the recordings and transcribed each video.

Following the transcription phase of the analysis, the videos were each watched a third time. This viewing was done with the transcriptions in hand. This served two purposes: we were able to verify the transcriptions were accurate, and we were also able to begin identifying aspects of the interviews that deemed relevant to the research. For the pilot study, this meant we were searching for interesting information regarding how students interacted with application problems requiring the definite integral. We also identified how students were able to progress through the pre-established layers of the RIF, if at all (Sealey, 2014). This analysis allowed us to refine the research questions that our study was able to answer. While performing the initial coding for interviews, we focused on annotating moments in the transcript that could relate to our research questions. In doing so, we began to see tendencies within and amongst interviews.

\section{Results}

As the students began working through problems related to definite integrals, it quickly became apparent that students experienced many discontinuities within their own definite integral concept image while attending to the limit layer of the RIF (Sealey, 2014). As a reminder, we began questions about the definite integral by asking students how they would find the exact area under a curve between a function's two roots. We followed this question by asking how an approximation would be constructed graphically since the algebraic representation of the function was not yet provided. Based on this approximation, the students worked on ways to make this 
approximation better, potentially reinventing the Riemann sum. This ability to transform an approximation using Riemann sums to an exact value was accomplished to varying degrees by the individual students. We will primarily discuss progression through the limit layer of the RIF starting with an understanding of how an approximation could be calculated. This will be followed by descriptions of how students improved upon this approximation.

Ultimately the final goal of obtaining an exact solution to the area under the curve will be examined. During the progression through this layer, there were a variety of levels of understanding. Some students had difficulty producing an initial approximation while others were able to almost completely conceptualize the abstract nature of this layer. It is important to note that in these results, students' demonstrations of understanding were dynamic as the interviews

Table 1: Summary of Limit Layer Understanding

\begin{tabular}{|l|l|l|l|l|l|l|l|}
\hline & $\begin{array}{c}\text { Nontraditional } \\
\text { Shapes to } \\
\text { Approx }\end{array}$ & $\begin{array}{c}\text { Reproduced } \\
\text { Riemann Sum } \\
\text { Graphically }\end{array}$ & $\begin{array}{c}\text { Improved } \\
\text { Graphical } \\
\text { Approx }\end{array}$ & $\begin{array}{c}\text { Explained } \\
\text { Why } \\
\text { Improvement } \\
\text { Was Better }\end{array}$ & $\begin{array}{c}\text { Technology } \\
\text { Bridged } \\
\text { Approx and } \\
\text { Exact Gap }\end{array}$ & $\begin{array}{c}\text { Discussed } \\
\text { Infinity in } \\
\text { Context of } \\
\text { Rectangles }\end{array}$ & $\begin{array}{c}\text { Extended } \\
\text { Approx to } \\
\text { Exact }\end{array}$ \\
\hline Arthur & & $\mathrm{Y}(\mathrm{O})$ & $\mathrm{Y}(\mathrm{NR})$ & $\mathrm{Y}(\mathrm{NR})$ & & & \\
\hline Chase & $\mathrm{Y}(\mathrm{O})$ & $\mathrm{Y}(\mathrm{NR})$ & $\mathrm{Y}(\mathrm{O})$ & $\mathrm{Y}(\mathrm{O})$ & & & \\
\hline Jamie & & $\mathrm{Y}(\mathrm{O})$ & $\mathrm{Y}(\mathrm{O})$ & $\mathrm{Y}(\mathrm{NR})$ & $\mathrm{Y}(\mathrm{NR})$ & & \\
\hline Jeremy & $\mathrm{Y}(\mathrm{O})$ & $\mathrm{Y}(\mathrm{NR})$ & $\mathrm{Y}(\mathrm{O})$ & $\mathrm{Y}(\mathrm{O})$ & & & \\
\hline Laura & $\mathrm{Y}(\mathrm{O})$ & & & & & $\mathrm{Y}(\mathrm{NR})$ & \\
\hline Oliver & $\mathrm{Y}(\mathrm{O})$ & $\mathrm{Y}(\mathrm{NR})$ & $\mathrm{Y}(\mathrm{NR})$ & & & $\mathrm{Y}(\mathrm{O})$ & \\
\hline Zoey & $\mathrm{Y}(\mathrm{O})$ & $\mathrm{Y}(\mathrm{NR})$ & $\mathrm{Y}(\mathrm{O})$ & $\mathrm{Y}(\mathrm{O})$ & $\mathrm{Y}(\mathrm{NR})$ & $\mathrm{Y}(\mathrm{O})$ & $\mathrm{Y}(\mathrm{NR})$ \\
\hline
\end{tabular}

progressed. Table 1 summarizes to what degree each student interviewed was able to progress

through various aspects of the limit layer. For context, "Y (O)" means the student was able to naturally demonstrate understanding of the particular aspect of a definite integral concept image. This is considered an original thought or idea. "Y (NR)" means the student demonstrated understanding, but that expression of understanding was a new revelation and was not initially apparent in the students evoked concept image. 


\section{Making an Approximation}

For some, like Jamie, it was easier to visualize a graphical representation by approximating the area under the curve using Riemann sums rather than using a definite integral to calculate the area exactly. She explained how, "the bar graph [referring to Riemann sums] makes so much sense to me when we were learning about the left-hand, right-hand and the area. When I look at this, my mind immediately goes there.” Rather than rely on newly acquired calculus concepts like limits and integrals, it appears that this particular student was more comfortable utilizing techniques and geometric shapes she had used in previous math courses. She became so fixated on bars that even 10 min later in the interview, she still was insistent on making Riemann rectangles. During this time, she progressed from using one large rectangle for an approximation (Figure 2a), to multiple rectangles with the same height (Figure 2b), to rectangles that closely resemble those that form a Riemann sum (Figure 2c).

Initially, even though Jamie drew one large rectangle for her approximation, she still stated that, "I would do left-hand side, right-hand side from one to seven, add them together and divide by two and that would be my area." Her use of recall of this LHS, RHS approximation process

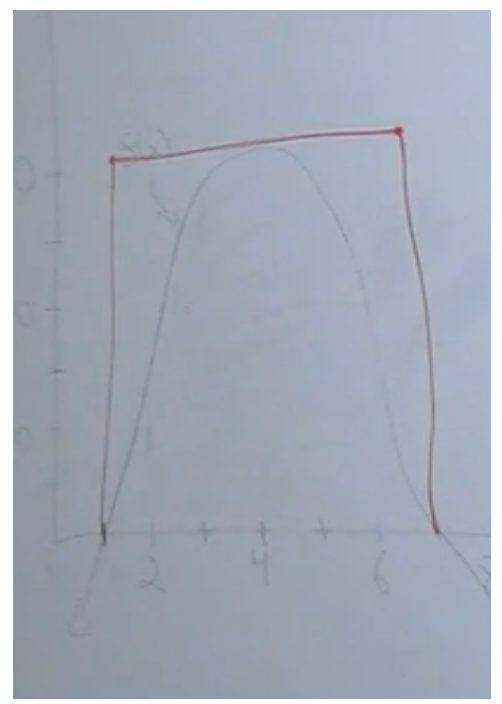

Figure 2a: Jamie initially used one rectangle

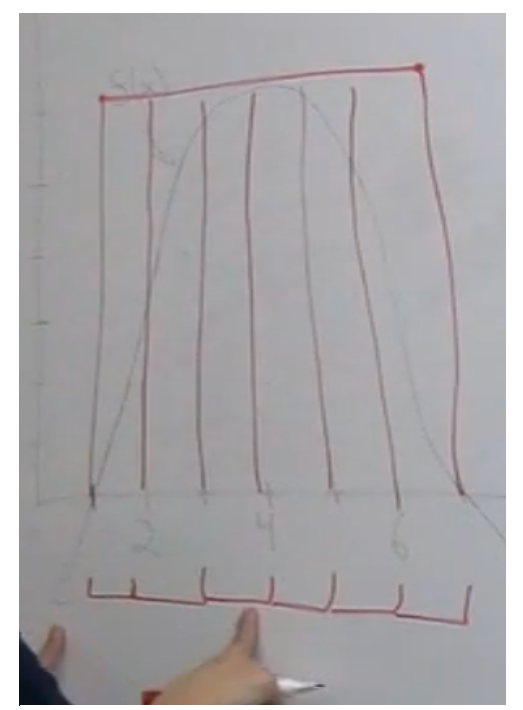

Figure 2b: Multiple rectangles of equal size

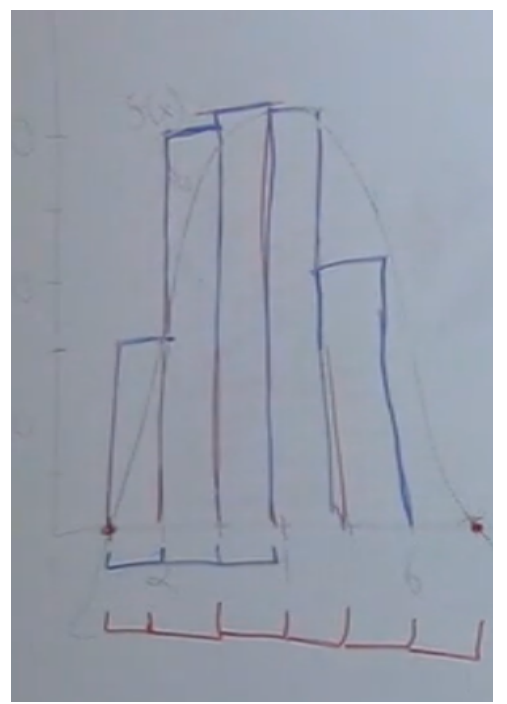

Figure 2c: Rectangles more closely aligning with a Riemann sum 
from class cued her that one large rectangle was not ideal. From here she made her 6 rectangles of equal height (Figure 2b). The next hurdle she had to overcome was understanding the rectangles would not all have the same height. After producing Figure 2b, the interviewer asked Jamie to compute the area. By successfully creating a RHS approximation computationally with a table, Jamie was able to deduce that the $f(x)$ values in her approximation represented the correct heights of each rectangle, resulting in Figure 2c.

While summarizing all of her progress, Jamie decided that the rectangles in Figure 2c were the best approximation of the three options because when summed, "it's closer to the area of what we're actually looking for, there is too much extra [area] that isn't part of [the curve]'. So, for at least Jamie, it appeared easiest to begin with concepts she had commonly used in the past. Through the use of trial and error, eventually she was able to correctly develop a graphical representation for an approximation that mimics that of a Riemann sum. For Jamie, the process of reinventing the Riemann sum relied on connecting the graphical representation to the numerical computation.

Not all students initially used the traditional vertical rectangles to approximate the area under this curve. In fact, five of the seven students first used shapes other than solely vertical rectangles to make this approximation. Chase decided to use horizontal rectangles stacked on top of each other (Figure 3a). Laura stacked rectangles horizontally and vertically, breaking the area up into a grid-like shape (Figure 3b). Both Jeremy and Oliver used triangles as well as rectangles to approximate the region. Zoey went as far as using triangles, rectangles, and semicircles to approximate this area (Figure 3c). We were very encouraged by the ingenuity of these responses because they indicated to us that creativity was being used, rather than simply recall cues. For consistency we discussed with the students that while these approximations were fantastic, we were going to proceed in the interview using only vertical rectangles. Zoey agreed with this 
constraint, reasoning that, "this is a lot easier because they're all rectangles. But also, you get left over with like this space that's outside of the equation and then this space is empty that's inside of the equation" (Figure 3d). So, while the calculations are easier with only rectangles, it was easy for Zoey to see how more error could result from this restriction.

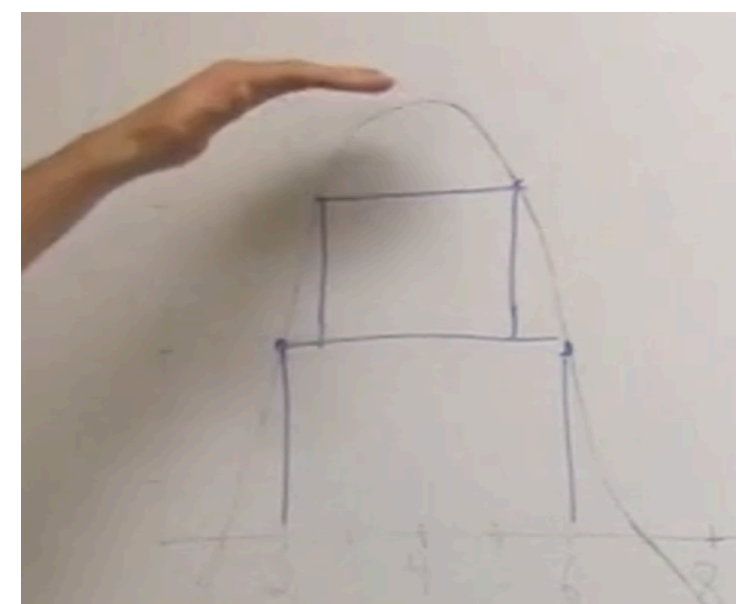

Figure 3a: Chase stacking rectangles vertically

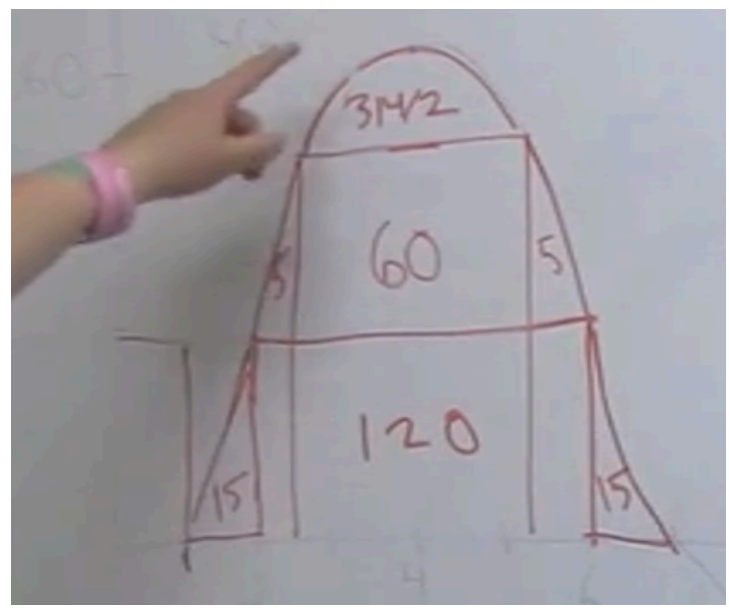

Figure 3c: Zoey using rectangles and triangles

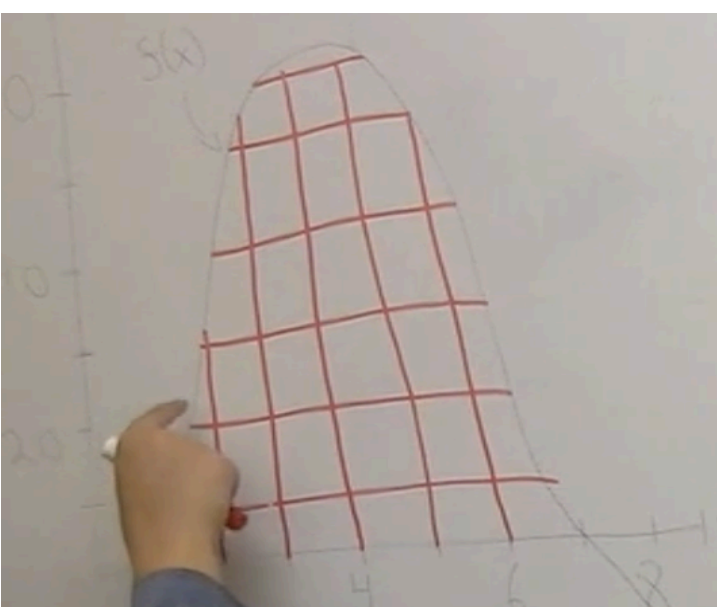

Figure 3b: Laura breaking region into grid

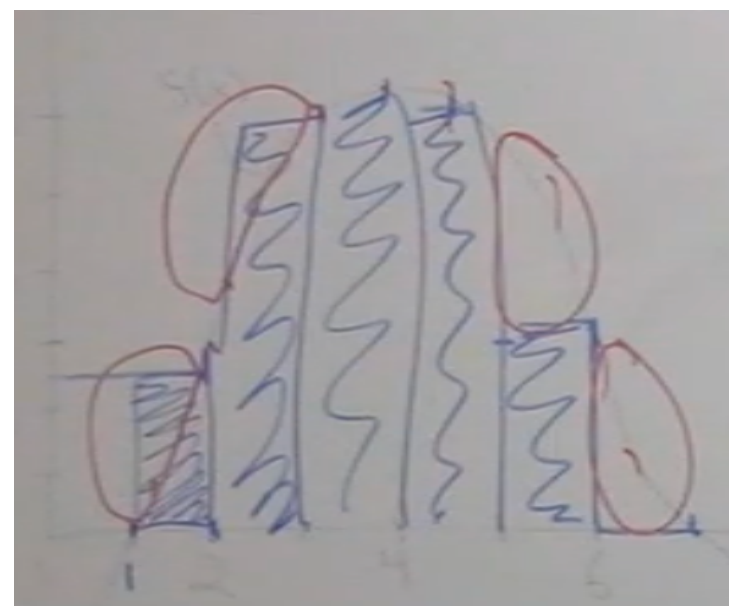

Figure 3d: Zoey identifying the cons of using only horizontally stacked rectangles

Jamie and Arthur also used geometric shapes to approximate the area under the curve, but their initial shapes more closely aligned to those of a typical Riemann sum. Already, these actions demonstrated that a major goal of the approximations was to use common geometric shapes that calculated the area under the nonstandard geometric figure. As some point, Oliver, Chase, Arthur, 
Jeremy, and Zoey were all able to identify that these geometric shapes did not perfectly align with the curve. This misalignment provided some sort of error either as an overestimation or an underestimation. All students but Laura were able to successfully draw six vertical rectangles to approximate the area under the curve (one of these rectangles had an area of zero so it was often omitted). Interestingly, tic marks on the $x$-axis were labeled, two, four, six, and eight, and yet every single student broke the interval from one to seven into six intervals of unit length one rather than three intervals of size two.

When asked how these approximations could be made better, only Arthur initially believed that his six rectangles represented both an approximate and exact area under the curve. Fortunately, by looking at these rectangles superimposed on the graph, Arthur was able to see that the area shaded in by these rectangles was in fact not the same as the area shaded in under the curve. Much like Jamie, Arthur was able to use the visual representation the graph provided to further draw conclusions in his reconstruction of a Riemann sum that may have otherwise been absent.

\section{How to Improve Approximations}

Once approximations were obtained by the students, a follow-up question asked how these approximations could become better. Jeremy quickly said, "I definitely believe reducing the size of each shape is going to give you a more exact answer." He then further explained that a "more exact" answer is still an approximation. Many other students were able to come to a similar conclusion, just described in a different manner. While Oliver, Arthur, and Zoey agreed that more rectangles resulted in a better approximation, Chase, Arthur, and Zoey all stated how smaller intervals would provide better approximations.

Zoey: The smaller you get, like the more accurate it would get. So, if you did 1.1, 1.2 or even smaller than that. The smaller, the more accurate it's going to be and the bigger, the less accurate it's going to be. 
Chase's rationale for the use of smaller intervals could be summarized as a reduction in rectangle widths. Rather than assuming the $y$-value at one spanned the entirety of the interval one to two, he could imagine using smaller widths, such as one to 1.5 . With this example, Chase explained that the smaller an interval a single $y$-value is required to approximate, the better the approximation will become.

Cody: So how would you make this approximation better or worse? Could you? Or is it always going to be the same?

Chase: No, I think this is going back to what I was saying earlier where you take one value and apply all the way across this range from 1.001 to 2.0. If you start breaking down into smaller intervals and get like 1.5 to two, two to 2.5 you'll start to get more accurate as you get more values.

While many of the students were able to state that smaller intervals and more rectangles resulted in a better approximation, Chase, Zoey, and Jeremy were able to give almost identical explanations why these changes result in better approximations. With guidance, Arthur was also able to produce a similar explanation. All four of these students described that when more intervals were used, the sum of errors produced by all of the rectangles was reduced. Arthur, Chase, Jeremy, and Zoey all explained their reasoning by

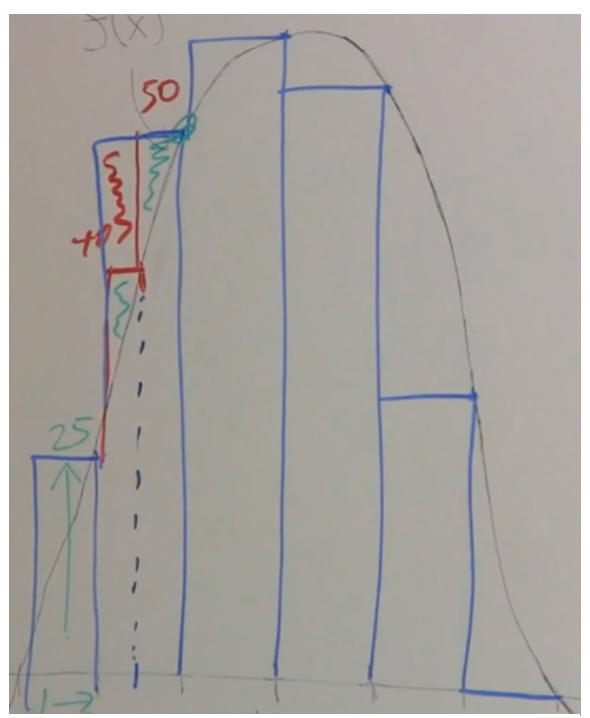

Figure 4: Chase explaining how smaller rectangles provide less error drawing smaller rectangles on top of the original rectangles and shading in the reduced error for the given interval, similar to Chase's representation shown in Figure 4. When looking at the second large rectangle in Figure 4, we can see two smaller rectangles contained within it. These two smaller rectangles represent Chase's explanation for why more rectangles resulted in less error. In his drawing, Chase was able to demonstrate that the two smaller rectangles resulted in an area that more closely resembled the area under the curve than the one large rectangle. Oliver was the only 
student who successfully demonstrated how to make his approximations better and never was able to express why this improvement was true.

\section{Difficulty Finding Exact Values}

Much more difficulty arose when students were tasked with expanding upon their improved approximations to obtain an exact calculation of the area under the curve. This disconnect between a finite number of rectangles to approximate and the limiting notion that leads to an exact value was demonstrated time and time again throughout the interviews. Jeremy, who said smaller shapes lead to better approximations, found great difficulty wrapping his head around the idea of making his shapes infinitely small. When discussing the idea of making approximations better to the point where it became exact, he, for the first time in the interview, expressed hesitation. By stating, "but I feel like once you get down to the nitty-gritty, like real tiny, it's going to be very difficult to get those areas and add them all up." Jeremy demonstrated the difference in difficulty between better approximations and obtaining an exact solution. This reluctance arose even after rationalizing that the $d x$ symbol in the definite integral represented the very small widths of the rectangles.

Earlier in the interview, Jeremy felt confident that the integrand could represent the height of his rectangles in his Riemann sum approximation. He was also able to confidently say towards the end of the interview that $f(x)$ and $d x$ were multiplying in the actual definite integral. In order for his concept image of these ideas to remain consistent, Jeremy rationalized that the $d x$ must also represent the width of the rectangles since the height and width of the rectangles also multiply.

Ultimately, Jeremy still demonstrated cognitive conflict factors because no matter how small this $d x$ became, there would still be at least some error in his eyes. After making such strong connections between the $d x$, the widths of the rectangles, and the reducing nature of the widths in 
order to obtain a better approximation, Jeremy's final thought was, "every single time you get smaller and smaller [referring to the rectangle widths], you're always going to have this curve making an awkward shape that you're not going to be able to get the area of."

In an effort to fit a square peg into a round hole, Chase tried to rationalize to himself that an exact value could be obtained by averaging a left-hand side and a right-hand side Riemann approximation and having their errors cancel. He quickly realized that this assumption was not necessarily true. Rather than explore other explanations for the extension of approximations to exact areas, Chase concluded that this idea of improved approximations could not be extended to an exact value.

Chase: I don't think you'll ever get it exact just because it's like the rectangles do have a width to them, so I think that prevents you from getting to that number along the curve. It will get you very exponentially close to it.

Oliver was able to build upon his reconstruction that more rectangles mean a better approximation to the point where an infinite number of rectangles may be used. But still, he did not believe this use of an infinite number of rectangles would result in the exact area.

Cody: So, do you see how when we use more rectangles it's a better approximation? How do we make it better so it's perfect?

Oliver: Keep increasing the [number of rectangles] to the nth term. I don't know what to put.

Cody: Let's see if we can stick to the idea. So just go ahead, even if we don't make it any better, just talk out loud to me about what you're thinking of why it's hard for you to get to it.

Oliver: If increasing the number of rectangles gives a better approximation then ... I don't know what the number of times it can increase this to get a better approximation.

Cody: Okay so how big can this get though?

Oliver: Big!

Cody: But how big though?

Oliver: Infinity...

Cody: So, we wouldn't want to draw an infinite number but it's more of a thought experiment. Do you think that if you had an infinite number of these it would be exact?

Oliver: No. 
At some point, Jeremy, Chase, Arthur, Zoey, Oliver, and Jamie all suggested that a better approximation of the area could occur, but improving this approximation could not lead to an exact area. Oliver and Jamie both believed that Riemann sum rectangles always give approximations while the definite integral gives the exact area. Zoey and Jamie both independently believed that the gap between the approximations using Riemann sums and the exact value is bridged by technology.

Cody: You said that you could have rectangles at like one, 1.5, and two to get a better approximation. Is there a way to get an exact value?

Zoey: Exact. My calculator does it for me.

Cody: What's happening that makes us switch from the integral symbol to our actual answer?

Jamie: The calculator works its magic.

The only person who transitioned from approximations to calculating the exact value with relative ease was, surprisingly, the only person who was unable to draw standard Riemann sum rectangles. Laura, who graphically represented her approximation by breaking the region into squares stacked horizontally and vertically (recall Figure 3b), immediately transitioned to an infinite summation when prompted to find the area exactly. The caveat, however, was that Laura used an infinite number of vertical lines, rather than rectangles to connect the ideas of approximations and exact area, making it still mathematically incorrect.

At no point in the interview did Laura identify the product layer of definite integrals. She instead was adding up "all of the [vertical] distances" to calculate the exact area (Figure 5). Prior to this, she explained that these "distances" were the length of the line from the $x$-axis to the curve from one to seven. The fact that the only student who readily made the

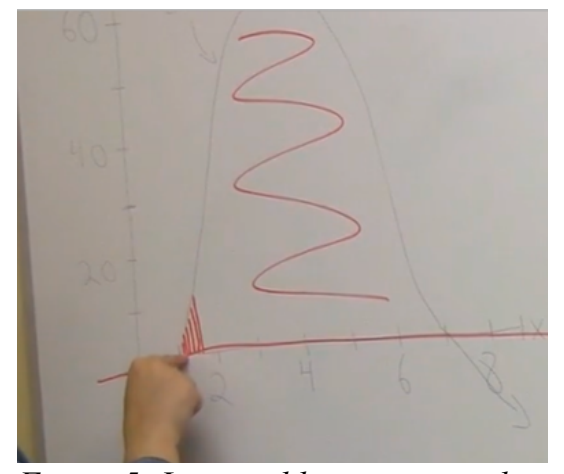

Figure 5: Laura adding up vertical lines, rather than vertical rectangles 
jump from approximation to exact calculations did not use rectangles could suggest that the finite width of rectangles causes a potential cognitive conflict when trying to understand the infinitesimal size of a differential. This potential hurdle in reconstructing a definite integral that resembles a Riemann sum was echoed by Jeremy in his reliance on assigning a finite value to $d x$.

Twenty-one minutes into the interview Zoey was able to understand the integral was "saying to do it with as many rectangles as possible." She clarified that, "I can do it with a lot, too, but it may be awful", insinuating that "as many rectangles as possible" was only a finite number. By the end of the interview however, Zoey no longer believed that a calculator was the only way to connect approximations to exact values. She was the only student of the seven interviewed to successfully expand upon the idea that smaller intervals and more rectangles leads to finding an exact value.

After 45 minutes of discussion, we asked Zoey the same question we asked at the start of the interview, "What about [the definite integral] matches each of these shapes", referring to the rectangles drawn for Zoey's original Riemann sum approximation. Zoey began by repeating from before that the $f(x)$ represented the height of the rectangles and the $d x$ represented the width. We then proceeded to ask what connections she could make between the approximations a Riemann sum provides and the exactness of a definite integral. She followed this question by attempting to summarize everything we had discussed but was not naturally evoked during the early stages of the interview. By doing so, we were provided insight into information contained in her concept image that was not previously apparent to us as the interviewers. She believed that the integral symbol means to sum up as many rectangles as possible, an infinite number. And this would ultimately result in an exact value. 
Zoey: I think that this [ $\left.\int\right]$ by itself would mean take as many as possible.

Cody: Take as many what as possible?

Zoey: Rectangles as possible.

Cody: Okay. And how many is that? How many is possible?

Zoey: I mean, technically, infinitely.

\section{Discussion}

For this area under the curve problem, great difficulty did not arise for the students in reconstructing the layers of the Riemann sum concept image until they began interacting with the limit layer. Once guided to use Riemann rectangles to approximate the area under the curve, students had little to no trouble developing a product to calculate the area of each individual rectangle and summing these rectangles up. Such ease while progressing through the product and summation layers of the Riemann sum concept image could be a direct result of the context in which the definite integral was being developed for this question. While others (Meredith \& Marrongelle, 2008; Sealey, 2014) have demonstrated student difficulty progressing through this product layer, we believe this difficulty is not independent of the problem's orienting pre-layer. For this study, the product layer was analogous to finding the area of a rectangle, a procedure we hope all students at this level of education are comfortable with. This ease likely is not present in scenarios where students are less familiar with the particular geometric or physical context (e.g., hydrostatic pressure or contexts of electromagnetism discussed in other literature). The readiness of a visual representation of the Riemann sum frequently cued students into ways of confronting potential cognitive errors they had. In another paper based on this data (Hood \& Sealey, in preparation), we further discuss how these students progressed through the product layer in a context other than area under a curve and what additional difficulties arose.

While only briefly discussed in this paper, students in this study aligned with preexisting research in their adept ability to progress through the summation layer. Even students like Oliver 
and Jeremy, who began calculating an approximation using nonstandard shapes, progressed through the summation layer flawlessly. Once done approximating the area of partitioned regions of the given area, every student knew instinctually to add these values together in order to obtain an approximation for the entire region. Furthermore, we posit that even the act of breaking the area into smaller components is evidence that students are entering the summation layer. While it is a very important component of the Riemann sum and definite integral structure, the summation layer is not a zone that should receive heightened attention when developing student understanding. This conclusion supports the point of view that less focus should be placed on computationally developing approximations. A student is provided the same opportunity for learning by improving an approximation using four intervals to eight as they would improving from using four to 64 intervals. This exorbitant increase in number of intervals may result in a student spending more time in the summation layer, when this research demonstrates the limit layer deserves heightened emphasis.

Using a variety of geometric shapes to approximate an area under a curve was a procedure that our students could perform with little to no assistance. They also generally possessed the ability to express why these shapes generated an approximation and why this was not exact. It became very evident that these results also support Sealey (2014) in that improving approximations of a Riemann sum comes with general ease for students. Being able to visually see the change in error these rectangles produce as more, smaller rectangles are used helps bring concrete images to an abstract idea. It is particularly interesting that this collection of students was able to progress through the beginning stages of the limit layer of the RIF because they were primarily provided a narrative reasoning in class of how approximations could be improved. This is done in contrast to many calculus courses that place a heavy emphasis on limit computations. 
Difficulty arose when extending the notion of improved approximations to finding the area exactly. Understanding that approximations could improve became relatively trivial early on in a student's understanding of Riemann sums. Extending a finite number of rectangles to an infinite number of intervals was where the majority of trouble occurred. Because of this distinction in the two stages of improving approximations and making an approximation become exact via the limit, it would be important for a teacher of calculus to distinguish between these two zones of potential struggle. Within Sealey's (2014) limit layer, we believed there were two sublayers: the approximation sublayer and the exact sublayer. In Sealey's (2014) study, very little attention was given to extending an approximation to an exact value. So, in essence, the approximation sublayer was analogous to Sealey's preexisting limit layer, while the exact sublayer adds to her original framework.

During the approximation sublayer, students attempted to understand how the sum of products that they previously worked with could have been improved upon. Again, many students seemed to thrive in their understanding that their $\lim _{n \rightarrow N} \sum_{i=1}^{n} f\left(x_{i}\right) \Delta x$ approximation improved as $N$ became larger. While some other students perceived this approximation improving by decreasing the size of $\Delta x$, the outcome of both types of understandings was the same. More rectangles over smaller intervals resulted in better approximations. Students in this study were able to proficiently demonstrate an understanding of this sublayer, even if it was not initially evoked. It is important to acknowledge that during this sublayer both $N$ and $\Delta x$ are finite in size in a student's concept image.

When the number of subintervals approaches infinity, the exact sublayer is entered. For this sublayer, the Riemann sum must be thought of as $\lim _{n \rightarrow \infty} \sum_{i=1}^{n} f\left(x_{i}\right) \Delta x$. While to many instructors, this jump from a finite to an infinite limit may seem like a small leap in knowledge 
acquisition, it is clear through this study and the research of others that students find great difficulty at this stage in their guided reconstruction (Cornu, 1991; Oehrtman, 2008; Oehrtman, 2009). Every physical model of the limit has these restrictions because it is impossible to perfectly represent the idea of infinity with physical representations (Oehrtman, 2009). When working with students learning about Riemann sums, it is important for instruction to distinguish between these two sublayers in a student's understanding. At face value it may appear that a student has not fully encapsulated the limit layer in his or her definite integral understanding. It would be a disservice to the student for a facilitator to end discussion and investigation into what the student understands here. By gaining insight into how much a student understands of this limiting process, one can better provide assistance to the appropriate region of conflict. Based on this study, confusion often does not begin in the limit layer until infinity and infinitesimals are required. This knowledge can and should help guide future instruction in a direction that helps build and strengthen these specific mathematical tools for students, rather than just assume that an individual is unable to grasp the concept of improving an approximation.

Now that it has been established that much concern in the limit layer of a Riemann Integral Framework derives from transitioning from the approximation to the exact sublayer, further research should focus on discovering what causes this intellectual hurdle. Some potential causes for this hinderance could be the above-mentioned difficulty for students to work and interact with infinitesimals and infinity. The disconnect could also be related to the common computational focus in calculus courses. For example, in the course the students were concurrently enrolled in, multiple lectures were spent discussing this idea of making approximations using a Riemann sum. However, once the theory behind improving an approximation to the point of obtaining an exact solution was presented, the FTC was used for any further exact calculations. Students may not 
fully grasp an understanding of the powerful link the FTC provides. Alternatively, students may become stuck in the approximation sublayer because there are many concrete visual tools that align with RME, such as physical rectangles, that provide a visual aid for this sublayer. It may be much more difficult to have a concept image that includes a visual representation for the exact sublayer because of its reliance on infinity. Now that it has been stated that there is a large disconnect for many students in the interior of the limit layer, it will be very interesting to explore why this disconnect may exist and ultimately how to overcome it.

Additional research is needed to help better understand how students can be guided through these two sublayers. This study was conducted in the confines of one specific calculus course at one university. These students were also never formally taught limits in this Applied Calculus course. It is important to ensure these findings can be supported in other settings as well. By better understanding this gap between approximations and exact calculations that exists in students' concept images, instructors will become better equipped to facilitate in bridging this divide. 


\title{
PAPER 2: DECONSTRUCTING SYMBOLIC COMPONENTS OF THE DEFINITE INTEGRAL TO ENHANCE STUDENT UNDERSTANDING
}

\begin{abstract}
There is currently great agreement amongst experts on the importance of students owning a definite integral concept image reflecting its definition. Here, we explore a potential pathway that guides students away from rote memorization and towards symbolically representing the layers that constitute a definite integral. Composing the definite integral are an integral symbol with bounds, an integrand, and a differential. Frequently, this collection of symbols is viewed as a single structure, giving direction to compute an antiderivative and to implement the Fundamental Theorem of Calculus. By having students dissect the definite integral into its components, they are more likely to be able to demonstrate an understanding of the definite integral that closely resembles a Riemann sum definition. In this research, we demonstrate this progress in understanding by presenting students with realistic applications requiring the use of a definite integral, and then having them break down symbolically why a definite integral results in the desired outcome. In doing so, we are able to elicit information students possess regarding the summation, product, and limit aspects of a Riemann sum definite integral that were not initially evident.
\end{abstract}

\section{Introduction}

The definite integral is a guiding mathematical topic for many second semester calculus courses. Comprising the definite integral are its bounds of integration, the integral symbol, the integrand, and the differential. Each component of this concept provides valuable meaning and understanding to its role in applications that require the use of an integral. Unfortunately, emphasis in the classroom is not always placed on the potential meaning and purpose behind each symbol. 
Commonly, students view the definite integral as a single entity, losing great allusions hidden amongst its parts. When viewed statically, the image a student owns of the definite integral has a strong likelihood of reflecting nothing more than rote memorization. The two most common ways the definite integral is viewed are as an instruction to perform the Fundamental Theorem of Calculus (FTC) and as a place holder that symbolically represents the Area Under a Curve (AUC).

By viewing the definite integral as nothing more than a directive to perform a computation, or as a static graphical representation, it can be argued that it would be nearly impossible for a student to possess a level of understanding reflecting the definition of the definite integral. Without the ability to understand the definite integral through a Riemann sum lens, deep understanding, which many experts (Jones, 2013; Jones, 2015; Meredith \& Marrongelle, 2008; Nguyen \& Rebello, 2011; Rösken \& Rolka, 2007; Sealey, 2014; Von Korff \& Rebello, 2012) believe is the most productive way to think about the definite integral, is impossible. Sealey (2014) has done extensive work with her Riemann Integral Framework (RIF) describing the process one can use to conceptualize the definite integral as an infinite summation of products. In this paper, when a student is able to view the definite integral through this arguably more prolific lens, it will be said that this student is demonstrating a Riemann sum concept image of the definite integral.

The Riemann Integral Framework is comprised of four layers and one pre-layer. We will be discussing ways students can explore and develop their understanding of three of these layers: summation, product, and limit. The symbolic construction of the definite integral will be the guiding tool to aid in eliciting this level of understanding from the interviewees. The traditional definite integral, $\int_{a}^{b} f(x) d x$, has the potential to provide great meaning to each of these three layers. To do so, we will draw parallels between the definite integral symbols, and its Riemann sum definition, $\lim _{n \rightarrow \infty} \sum_{i=1}^{n} f\left(x_{i}\right) \Delta x$. The integral symbol could possess information instructing us 
to perform a summation over the interval $a$ to $b$. The product layer, symbolically represented by the integrand and differential multiplied together, can be seen as analogous to the multiplication in $f\left(x_{i}\right) \Delta x$. Additionally, the $d x$ in the integral could potentially embody the $\Delta x$ in the Riemann sum once the limit to infinity is applied, making it infinitesimally small and introducing the limit layer in the process. Here, we are not asserting that this potential meaning is intrinsically required in the definite integral. Instead, we are suggesting that if a student were to apply such meaning to the individual symbols that comprise the definite integral, they would possess a greater ability to understand the definite integral with a Riemann sum concept image.

In this study, we observed how Applied Calculus students were able to attribute meaning to the integrand, the differential, and their interaction in the context of a definite integral. This analysis was achieved via the aid of visual tools in the context of calculating an area under a curve and an application prompt involving a fire spreading through a house. Students were first given a graph and were tasked to recreate the Riemann sum definition for the definite integral through the use of geometric and graphical approximations of the area under the given curve. The goal of this exercise was to help elicit connections and develop meaning that related the Riemann rectangles and the calculation of their areas to the components of the definite integral. Following this discussion, students were presented with a real-life application involving a fire spreading through a house at a given rate. They were tasked to calculate the total amount of house engulfed by flames at a given time. Our research indicated that the use of a graphical representation that resembled a Riemann sum and the use of a realistic application that utilized true-to-life units provided positive results. Students were given the opportunity to deconstruct the components of the definite integral to develop their understanding of the mathematical concept through a Riemann sum lens. Through this paper, we addressed and answered the following research questions. 
1. What relationships exist between calculus students' concept images of a Riemann sum and the symbols used to construct a definite integral?

2. How can the units of a realistic definite integral application aid in guiding students through the Riemann Integral Framework?

\section{Literature Review}

Tall and Vinner (1981) introduced the mathematical community to an explicit way of categorizing knowledge a student has about a particular subject matter. "[They] use the term concept image to describe the total cognitive structure that is associated with the concept, which includes all the mental pictures and associated properties and processes" (Tall and Vinner, 1981, p. 152). A student's concept image is a forever growing and changing construction that adapts to newly acquired knowledge as he or she becomes presented with new or expanding ideas. An individual's concept image consists of not just a formal definition, but also visual tools, examples, nonexamples and informal definitions just to name a few. In turn, these concepts images, along with newly acquired information, serve as foundational building blocks for future concept images this individual will inevitably develop. Because many aspects of math are sequential and iterative, it is important to note that a person's concept image of a particular idea does not stand alone, but rather is incorporated and intrinsically tied to other concept images of topics this individual possesses, creating a web of knowledge (Thompson, 1994).

While concept image is used as the guiding theoretical perspective for this paper, we briefly described it now because its terminology is frequently prevalent in the literature review. Here, we will discuss different types of concept representations students may possess in regard to the definite integral and how certain ones may be more prosperous than others. We will begin with an understanding that reflects the definite integral's formal definition. This will be followed by a 
discussion involving two of the most common, yet less prolific depictions students often utilize. We will describe some common drawbacks of each image, as well as misconceptions that could develop or exist as a result of solely relying on only one of these images. It is important to state that at any moment a student's actual overall concept image may not be well reflected by his or her evoked concept image in a given context.

\section{Riemann Sum Concept Image}

While many formations of a concept image can exist, it is collectively agreed upon that throughout mathematics, an image closely aligning with a formal definition is the most prosperous one (Jones, 2013; Jones, 2015; Meredith \& Marrongelle, 2008; Nguyen \& Rebello, 2011; Rösken \& Rolka, 2007; Sealey, 2014; Von Korff \& Rebello, 2012). It is essential to reiterate that a concept image goes well beyond a formal or even an informal definition. Simply put, it is important to have a formal definition of a concept image involved in the formulation of a given concept image's foundation. Unfortunately, for many students in calculus, the formal definition of the definite integral, grounded in the Riemann sum definition, seldom exists in their evocable concept image (Doughty, L., McLoughlin, E., \& van Kampen, P., 2014).

Throughout definite integral education research, there have been many ways this concept image, grounded in its formal definition, have been described. Some of these descriptions are referred to as parts-of-a-whole, an accumulation of rates, adding up pieces, and multiplicatively based summations (Jones, 2015; Rösken \& Rolka, 2007; Sealey \& Thompson, 2016). While each method of describing definite integral understanding has their slight differences, they all share the commonality of being rooted in a Riemann sum interpretation of the definite integral, $\lim _{n \rightarrow \infty} \sum_{i=1}^{n} f\left(x_{i}\right) \Delta x$. We will be referring to this level of understanding of the definite integral as the Riemann sum concept image. 
In order to decompose the Riemann sum concept image into manageable and impactful components, we will be using Sealey's (2014) Riemann Integral Framework (RIF). The RIF breaks the definite integral into five parts, one pre-layer and four layers. In Sealey's (2014) framework, students must first orient themselves with the context of the given problem to rationalize what information the question is actually trying to communicate. In this pre-layer, students are familiarizing themselves with the problem by, "understanding what each quantity represents in the problem context" (Sealey, 2014, p. 237). In addition to understanding the necessity of integration for a given application, a student must also be able to successfully identify the multiplication of which quantities is occurring. This multiplicative understanding enters the student in the product layer of RIF.

Students typically are able to instinctively enter Sealey's (2014) summation layer by adding up each product over a set of intervals in order to obtain an approximation of the desired result. Unlike the ease associated with the summation layer, difficulty ensues in improving upon this approximating technique to eventually calculate an exact result (Sealey, 2014). The required incorporation of previous knowledge associated with the limit, historically causing students great difficulty, to improve this approximation to perfection warrants substantial concern (Orton, 1983).

The limit layer is the section of the RIF that takes this approximation and makes it exact. In this complex layer, a leap in understanding is required to expand upon finite notions by incorporating limits and infinity. In this layer, students are required, sometimes for the first time, to think abstractly to reduce a finite $\Delta x$ to an infinitesimal differential (typically $d x$ or $d t$ ). This layer can be broken down into two phases. First, students must understand how to take the approximation they originally developed and use smaller interval sizes improve the approximation. This phase is relatively easy to understand in comparison to the second phase, which takes the leap 
of improving an approximation to a point where an exact calculation occurs (Hood \& Sealey, in preparation). Many students rely on memorizing the FTC to bridge the gap between approximations via Riemann sums and exact calculations described by the definite integral (Sealey 2014). Her research, and others, suggests that while students know an exact calculation exists and how to reasonably improve upon their current approximation using smaller interval sizes, the link connecting the two ideas is shrouded in mystery for many students (Jones, 2013; Meredith \& Marrongelle, 2008; Sealey, 2014; Hood \& Sealey, in preparation). If the limit concept image is not developed adequately, it is unlikely that a student will possess the ability to extend past the rationale that smaller rectangles will result in a better approximation. If a student were able to incorporate an infinitesimal representation of the differential into their understanding of the definite integral, it is likely that a greater connection to the limit layer would exist as well. By assigning infinitesimal language to the differential, this provides students with the opportunity to draw direct correlations to the collapsing nature of delta $x$ in a Riemann sum, and how this concept could collapse small enough into $d x$.

The fourth layer of Sealey's (2014) RIF is the function layer. This layer requires a student to be able to view this definite integral dynamically rather than statically. Thompson supports these claims by emphasizing the importance of "thinking of quantities as being composed multiplicatively of two other quantities, and thinking of terms of infinitesimals" (1994, p. 132). Taken together, the pre-layer and subsequent additional four layers combine to yield a substantive and imposing understanding, reflecting the definition of the definite integral, which states $\lim _{n \rightarrow \infty} \sum_{i=1}^{n} f\left(x_{i}\right) \Delta x=\int_{a}^{b} f(x) d x$ (Sealey, 2014).

Because of its complexity, this Riemann sum interpretation of the definite integral provides the opportunity to evoke many connections between its components and a given application. The 
product, summation, and limit layer of the definite integral all have the opportunity to be intrinsically tied to the symbols that construct the definite integral and their interaction (Jones, 2013). The $f(x)$ and $d x$ interaction elicits connections to the product layer. The integral symbol itself could reflect the summation layer of the definite integral. The differential encapsulates the limiting and dynamic nature of the definite integral by incorporating an infinitesimal size. This connection would be likely unachievable if a student's concept image of the definite integral only consisted of static images and rote memorization (Meredith \& Marrongelle, 2008). By putting in the work initially to understand the definite integral from this Riemann sum perspective from its inception, a student has a much greater chance of being able to identify when a particular application calls upon its use.

\section{Two Common Representations}

While a Riemann sum concept image is collectively understood as the most prosperous way of understanding the definite integral, students frequently rely on concept images that closely mimic the simplicity of rote memorization when interacting with definite integrals (Doughty et al., 2014; Jones, 2015; Nguyen \& Rebello, 2011, Rösken \& Rolka, 2007). From the student's perspective, it is understandable that they choose an elementary representation. This is even more true when such a remedial level of understanding is all that is required to be successful in a calculus course. When the focus of the definite integral is solely its evaluation, the meaning and purpose of each aspect of the definite integral and its notation is severely limited or even obsolete (Doughty et al., 2014; Thompson, 1994).

For clarity, we will be partitioning this common perception of the definite integral into two categories. The first reflects an understanding mimicking the Fundamental Theorem of Calculus (FTC). Especially in math courses where the application of the definite integral does not have a 
large emphasis, the main outcome of a definite integral in the eyes of the student is numeric manipulation to obtain a solution (Hall, 2010; Jones, 2015; Nguyen \& Rebello, 2011). The integral is seen as nothing more than an assertion to perform a computation. As a result, having a concept image of the definite integral that mirrors the FTC, which states $\int_{a}^{b} f(x) d x=F(b)-F(a)$ where $F(x)$ is the antiderivative of $f(x)$ often is sufficient in obtaining correct answers. With this concept image, the definite integral is reduced to a function matching game between the given function and its antiderivative (Jones, 2013). By "introducing integration as a rule, as antidifferentiation in fact" (Orton, 1983, p. 10), a lack of understanding concerning the individual components that make up the definite integral is created.

The second common image associated with memorization is reducing the definite integral to a static graphical representation, Area Under a Curve (AUC) (Doughty et al., 2014; Jones, 2015; Nguyen \& Rebello, 2011, Rösken \& Rolka, 2007). The area under the curve's ability to provide students with an easy-to-understand fixed visual representation of the definite integral leads to it having such a strong presence in many concept images. Following the FTC concept image, the AUC is the second most common image students in calculus possess of the definite integral (Jones 2013). We believe this image of the definite integral is often called upon by students because of its ability to bring some visual meaning to an abstract idea. Understanding the definite integral as an area under the curve can help students understand relatively simplistic applications, but this approach can fall short in aiding the understanding of applications that cannot be represented easily two-dimensionally (Nguyen \& Rebello, 2011; Sealey, 2014; Thompson, 1994). Just like with the FTC concept image, the AUC concept image is an oversimplification of the definite integral that allows for quick connections to be made but can ultimately fall short in providing the necessary tools to successfully conquer less routine applications. 


\section{The Need for Multiple Representations}

“[A] student's concept of integration may not be a single entity, but rather be made up of smaller units, including ideas of area, anti-derivatives, summations or differentials" (Jones, 2013, p. 123). This incorporation of different representations into a single concept image provides students with an ability to view definite integrals from multiple perspectives, reducing the likelihood of misconceptions (Doughty et al., 2014). Because of this, it is important to, again, discern the difference between a student's concept image and a student's evoked concept image. Just because a student may not demonstrate knowledge of a particular concept image in a given context, it cannot be assumed that the student does not possess that particular image (Jones, 2013).

\section{Theoretical Perspective}

To guide the research in this paper, we primarily rely on Tall and Vinner's (1981) concept image. This work on understanding a student's concept image will be supplemented and uncovered using Gravemeijer and Doorman's (1999) tools of Realistic Mathematics Education (RME). The research presented in this paper strives to contribute and expand upon how students understand the definite integral in an application context. This goal is achieved by presenting students, in an interview format, applications requiring the use of an integral that incorporate tangible, realistic scenarios.

We pay particular attention to discerning between an individual's concept image and his or her naturally evoked concept image. An individual's concept image is not a static entity. It is constantly expanding, developing and being modified as a person encounters new situations (Meel, 2003). Thus, our research focuses on ways these application problems, through moderate guided reinvention, can help evoke powerful ways a student can interpret and interact with a definite integral. 


\section{Understanding Concept Image}

Every concept in mathematics is rigorously defined by a formal definition. This, often concise, definition technically contains within itself all the information needed to encapsulate the idea fully. In practice, this is not the case. Students and educators alike use a variety of ways to think about, rationalize, and describe a given concept that seldomly resembles a strict adherence to the concept's formal definition. This collection of thoughts and ideas an individual possesses about a particular topic is called a person's concept image (Tall \& Vinner 1981). Contained within this image is a personal concept definition, which relies on an individual's definition, rather than one necessarily universally accepted by the mathematical community (Davis \& Vinner, 1986; Meel, 2003; Tall \& Vinner, 1981). This understanding may or may not have connections back to the concept's formal definition. In fact, in many undergraduate courses, students can associate ideas with a concept without even knowing or understanding what its formal definition is.

While interacting with a new concept, students continually add, remove, and modify information that they associate with it as they are presented with more data about the concept. Importantly, a student's concept image, which is unique to that individual, contains more than just an informal or personal definition. A concept image can contain symbols, ideas, formal definitions, personal definitions, visual representations, and varying examples that an individual associates with a given concept. The concept image, "consists of all the cognitive structure in the individual's mind that is associated with a given concept" (Tall \& Vinner, 1981, p. 151)

Not surprisingly, these concept images often contain contradictions while forming. From these inconsistencies arise conflicts. When being introduced to a new concept, a student's understanding is constantly molding and changing to fit the context at the present moment. The idea that potential conflict always exists but has yet to be evoked is called the potential conflict 
factor (Tall \& Vinner, 1981). These factors are inconsistencies that are present in a person's concept image but have yet to be brought to the individual's attention. Once a person encounters a contradiction in his or her current concept image, the potential conflict factor becomes a cognitive conflict factor. The individual is now tasked with the mission to overcome this conflict to no longer have an elicited self-contradictory image. These conflicts can be as blatant as conflicting assumptions or something as minuscule, "a vague sense of unease" (Tall \& Vinner, 1981, p. 154). Identifying and resolving conflicts is an important method to improving a personal concept image, leading to a better understanding of the mathematical concept. As previously stated, the difficulty arises not just in resolving these conflicts, but self-identifying them as well. Potential conflicts can only be overcome if an individual realizes that a contradiction is present (Tall \& Vinner, 1981). Even with the help of an instructor, identifying these conflicts can be difficult because only evoked concept images, a small subset of an individual's entire concept image, can bring these inconsistencies to light (Doughty, McLoughlin, \& van Kampen, 2014; Tall \& Vinner, 1981). If an individual possesses a potential conflict factor but is never presented with a situation that draws its attention, it is understandable that the individual will never be able to improve upon his or her own concept image to alleviate this concern.

One must be careful not to assume that a person's evoked concept image is a complete or even valid representation of his or her complete concept image. A major goal of our research was to understand this distinction between evoked concept images and what knowledge a student actually possesses. It is important for someone to be able to successfully elicit and use appropriate tools to understand the necessity of a definite integral. However, if a student fails to naturally evoke this knowledge, it is important to discover if he or she still possesses the information but just not the proper tools to evoke it. By identifying these conflicts, not only is a student able to 
improve upon his or her current concept image, but it also distinguishes between the ability to possess knowledge and the ability to evoke it.

\section{Realistic Mathematics Education (RME)}

Gravemeijer and Doorman (1999), researchers at the forefront of RME, believed that with the aid of the environment, students are able, "to (re)invent the more formal mathematics" (p.159). The use of the word (re)invent attempts to illustrate the balance between the two sources of knowledge described by emergent constructivism. Guided reconstruction allows for students to make connections between mathematics and their own reality that contain an aspect of ownership and individualistic pride in accomplishing that ownership (Gravemeijer, 1999; Gravemeijer \& Doorman, 1999; Hough \& Gough, 2007; Treffers, 1993). A metaphor Prawat and Floden (1994) have for the emergent theory expresses the idea that truth unfolds, given a context. Emergent theory is rooted in the belief that truth emerges from individual creation and environmental discovery.

RME, developed by the Fruedenthal Institute in The Netherlands, uses models to convey nonabstract situations as an avenue to learning potentially abstract mathematical concepts. In our research, we used RME to allow for an informal presentation of mathematics in the fashion of realistic situations that an individual could use to construct a personal interpretation of more formal mathematics (Gravemeijer, 1999; Gravemeijer \& Doorman, 1999; Hough and Gough, 2007; Treffers, 1993).

This process of learning using an RME mode of instruction is a complete role reversal of many traditional learning environments. Throughout schooling, mathematical concepts are often first presented formally. Through exercise and practice, students then develop an informal representation of the formally taught concept to help further understanding. In contrast, when 
students gain familiarity with a concept initially through the use of a real-life situation, they can then begin to develop a formal understanding of the concept with which they are already familiar (Gravemeijer \& Doorman, 1999; Treffers \& Beishuizen, 1999). These realistic models are the key feature that Fruedenthal, the founder of RME believed provided the opportunity for true learning and knowledge acquisition to occur (Treffers, 1993).

\section{Methods}

\section{Data Collection and Interview Subjects}

The students being interviewed for this study were concurrently enrolled in Applied Calculus at our university. This course differed from many standard calculus courses in two main ways. First, heavy emphasis was placed on the application of calculus concepts. This stress on applications was done so in lieu of a computational focus many calculus courses have. While basic derivative and integral rules were learned, some more difficult methods, such as implicit differentiation and integration by parts were omitted. In addition to this, no rigorous definition of the limit was presented in class. Instead, heavy focus was placed on real-life application contexts of derivatives, integrals, and limits. The second way this course stood out was the order in which new material was introduced. Students were first presented with a scenario that warranted the necessity of a calculus idea, and then from this necessity, the concept was discussed, followed by a more rigorous presentation.

This model mimicked RME in its heavy reliance in understanding the purpose of mathematical concepts before formally introducing them. For example, prior to seeing any integration techniques or even Riemann sums, students were given a scenario where they knew the speed at which a vehicle is traveling and wanted to determine how far the vehicle had traveled. Students were then able to discover how to approximate total distance traveled using given 
speedometer readings, and also how to improve upon this approximation. In presenting the material in this manner, students were able to reconstruct the product, summation, and limit layer of a definite integral with only a limited background in the content. Instead of being presented with a formal definition of the definite integral, students were first given a situation they may be familiar with and used this to develop an understanding.

Students in this course were also provided the opportunity to use calculators to determine the numeric value of a definite integral. Heavy emphasis in this course was placed on understanding why any integral was necessary in applications, so once demonstrating an $80 \%$ level of mastery in calculating antiderivatives, students were permitted to use calculators to compute definite integrals for the remainder of the semester. Again, this was done to divert emphasis away from computational understanding and place it on understanding the applications of the concepts.

One visual application these students became familiar with was the area under a curve representation of a definite integral. Students in this course had seen what a Riemann sum approximation looked like graphically as a summation of rectangles, and it was discussed how these approximations could be improved upon. The culmination of the graphical aspect of this unit was understanding that a Riemann sum could approximate the area under a curve and the definite integral computes this exactly.

Prior to the interviews, students had already completed a majority of the definite integral unit in class. All that remained for these students were some of the more advanced applications involving income streams, present value, and future value. During class, students were asked by their instructors to participate in our study. The recruitment script the instructors read to the classes divulged that the interviews involved material covered in class, but nothing more pertaining to the 
actual content of the interviews. An additional email was sent out to recruit students as well. The students received no compensation, monetary or otherwise, for their participation.

Students who had elected to participate were reminded via email that interviews would be videorecorded and were asked to sign up for an interview time. Subjects who attended their interview time were given the consent form and an opportunity to ask any questions they may have had. Videorecording began once subjects signed the consent form. Pseudonyms were used in place of real names to keep student information confidential. Gender, ethnicity, race, age, sex, and class ranking had no impact on this study, and no data on this criterion was collected.

The interviews ranged from 40 to 50 minutes in length. In addition to some handouts, the students were also presented with a whiteboard to write down any thoughts they may have had. We continually tried to encourage the interviewees to write as much as they could in regard to what they were thinking in order to better capture as much of their evoked concept image as possible.

\section{Interview Protocol}

While the interview protocols were designed to be strictly adhered to, they were created to allow for flexibility on the researchers' part. The interviews were conducted in a way that allowed the interviewer to ask follow-up questions that were dependent upon student responses and insights given when it was warranted. Follow-up questions were also used when additional clarification was needed, when a response was found particularly interesting, or when a comment contradicted a previous statement by the student. These additional questions by the interviewer were intended to guide students to rediscovering the connection that exists between Riemann sum approximations and how they can be used to define a definite integral. As a result of time constraints, questions or parts of questions were omitted from some interviews. 


\section{Question 1: Regarding Area Under the Curve}

We began each interview by providing the following graph in two formats (Figure 6). To the best of our ability, we accurately drew this graph on the whiteboard to encourage student interaction. We also provided students with a printout of the graph to ensure no details were lost through hand-drawing. Since one of our research goals involved students using Riemann sum rectangles to gain meaning behind $\int_{a}^{b}, f(x)$, and $d x$, we wanted to use a function that allowed for the construction of multiple rectangles with a nonzero, positive area. If a student decided to use rectangles with a width of one for this function, five rectangles with a positive area would exist. A sixth rectangle with an area of zero may or may not have been included as well.

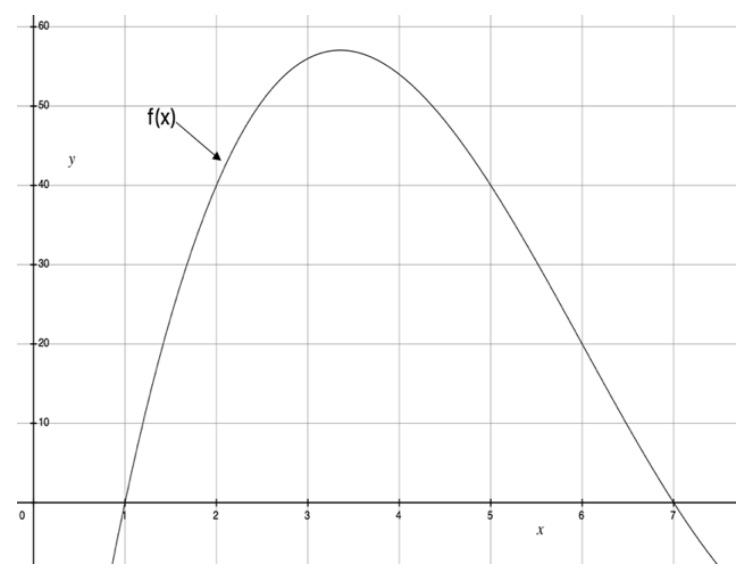

Figure 6: Graph of $f(x)=x^{3}-18 x^{2}+87 x-70$

Once the students were provided with this graph, they were asked, "How would you go about finding the area of the following region above the $x$-axis?" Most of the students interviewed knew to some capacity that a definite integral was required to calculate this area. Based on their response we followed up this question with, "How do you know that you are correct?" Once they discussed their rational for the necessity of an integral, we went symbol by symbol and asked what each aspect of the definite integral notation referred to graphically, if at all. In addition, if any aspects of the traditional $\int_{1}^{3} f(x) d x$ were omitted when the student demonstrated the need for an 
integral, we made note that some other people believe the definite integral should include this symbol as well. As an example, if a student were to write $\int_{1}^{3} f(x)$, we would follow up by saying, "Others have said a $d x$ is needed here. Is there a reason why you didn't think it needed to be included?"

After the definite integral was set up, we asked for an estimation of this area. The actual calculation was not a main point of interest to us. Instead, this addition to the protocol helped us better understand what method a student would use to find an approximation. In doing so, interviewees were encouraged to geometrically approximate the area. At this point in the interview, the actual equation of the resulting graph was not yet given, forcing students to rely on techniques other than the FTC to gain a numeric answer. If the given approximation used shapes that did not mimic Riemann sum rectangles, we attempted to guide the student to this method of approximation to encourage consistency and to hopefully provide a greater opportunity to make connections between the approximation and the definite integral.

Students were then asked how to make this approximation better. A primary outcome we attempted to achieve from this next question was a connection between the individual symbols that comprise the definite integral, the Riemann sum, and area under the curve. We also sought to understand to what degree students were able to reconstruct a Riemann sum understanding of the area under a curve. It is important to reiterate that one of the major goals of our study was to determine what concept images students have the ability to possess/develop, not just which ones are naturally evoked like much of the existing research has done thus far. We were not interested in discerning between newly constructed understanding and elicited understanding. We were interested in what understanding could be present by the end of the interview that was not initially evoked. In taking such an approach, we were able to move past the notion that students typically 
memorize situations in which a definite integral is needed to have these students work towards applying meaning to a process they knew was required.

This improvement in approximation was then expanded upon once more to see if students possessed the ability to fully encapsulate the limit layer of the RIF by theorizing how an exact solution would be obtained. Our goal was to better understand how these students progressed through the varying layers of a Riemann sum and how understanding could be developed by improving upon a graphical approximation to eventually obtain an exact answer. A major distinction and potential conflict factor here existed in the relationship between a shrinking, but still finite $\Delta x$ and the infinitesimal nature of $d x$.

The overall goal of these interviews was to see how well Applied Calculus students could deconstruct and apply meaning to the components of the definite integral. It was very promising to see the varying levels of success students were able to have while being guided through the development and necessity of the individual symbols needed to construct a definite integral in the context of area under the curve. The goals of our research questions were accomplished by asking students not only how an area under a curve could be numerically computed, but also why the given construction worked and what its parts represented. Ultimately, we worked towards having these students complete the nonlinear journey through the Riemann sum layers by developing an approximation for the area under a curve, improving this approximation, and eventually connecting to the definite integral and an exact value.

\section{Question 2: Rate, Accumulation Application}

The second question we asked the research participants moved away from a graphical representation of the definite integral and towards its application in a real-life context. The following prompt and question were provided to the students. 
A fire starts in a 5000 square foot house at midnight and spreads at a rate of $f(t)=e^{\frac{t}{2}}$ square feet per minute, $t$ minutes after the fire begins. How would you calculate the amount of the house engulfed by the fire at time a?

This particular scenario was selected because it holds many similarities to questions the students should have been familiar with from their Applied Calculus course. They had interacted with questions involving the rate at which a wildfire spread. We adapted this question to a similar scenario and included additional information, a 5000 square foot house, in hopes to elicit varying potential cues for when a definite integral is required.

As we did with question one, once students provided us with an answer, whether correct or incorrect, we asked them how the students knew they were right. In this example, because students were much less likely to presume a definite integral was required, the interviewer frequently asked what units were given in the problem and what units did the question ultimately ask for. This redirection by the interviewer provided many students the opportunity to recognize the mistake of simply plugging $a$ into the given function to obtain an answer.

Following students discovering the necessity of a definite integral, we asked what each aspect of the definite integral meant. Again, this was done in hopes of eliciting the layers of the RIF including product, summation, and limit. If not done so already, we also asked the students what units would make sense for the definite integral and what aspects of the definite integral could be used to discover such a conclusion. This question was included in every interview conducted because of the potential it allowed to elicit the product layer of the Riemann sum concept image. If a student was able to view the definite integral as a summation of products, then this student had the potential to use the units of the product to determine the units for the resulting definite integral. This idea of using the symbolic structure of the definite integral to determine its 
units was revisited at the end of the interview by providing obscure $x$ and $y$ units and asking what the units of the definite integral would be and why.

Once we had a strong understanding of how these students oriented themselves with the problem at hand, we circled back to the original question regarding area under a curve. Now that the students had been presented with a second application requiring the definite integral, we wanted to see if any further connections between the definite integral symbols and its graphical representation existed. Students now possessed the tools of a real-life application, as well as potential connections regarding units to help expand upon the symbolic relationship between Riemann sum rectangles and the definite integral. Ultimately, the goal of this follow-up question what to culminate the thoughts and ideas students had expressed and modified throughout the interview process. This gave students an opportunity to address unease they previously expressed and to solidify rationalizations they developed earlier in the interview.

\section{Data Analysis}

The primary purpose of our analysis was to understand how students were able to apply meaning to the components that comprise the definite integral in a variety of applications that require the use of a definite integral. Careful attention was given to distinguishing between which of this information was naturally elicited and which was divulged through the use of guided reinvention. The units involved in the given applications proved to play a large role in this analysis. A secondary goal of our analysis was to determine how current literature on student understanding of the definite integral aligned with the population group of Applied Calculus students with whom we worked. 


\section{Thematic Analysis}

Once the interviews had been completed, we analyzed the data using Braun and Clarke's (2006) thematic analysis for qualitative analysis to answer our research questions. In doing so, we familiarized ourselves with the data collected, generated, identified, defined, and connected themes within and across interviews. With these steps of thematic analysis guiding us, we were able to take our data, in the form of video recordings, transcriptions, and handwritten notes, and develop a communicable understanding of how students applied meaning to the varying elements of the definite integral. As an aid to conducting thematic analysis, Strauss and Corbin's (1998) method of axial coding was used to decompose and support the themes we discovered. This was the primary technique we utilized to help identify and confirm themes that existed across research participants.

An inductive, rather than theoretical, approach was used to discover what themes were contained within the data. One reason this type of thematic analysis was chosen was to help ensure the results were unique to this study, and themes were not biased by preexisting research. The data, rather than hypotheses by the experts, guided this development of themes.

\section{Coding Data Points}

Each interview was watched and timestamped for any information that had the potential to be relevant to our research questions. This included information that related to the layers of RIF, the three common concept images of the definite integral, the impact concentrating on units had in eliciting a Riemann sum concept image, and any information regarding student understanding of the symbolic construction of the definite integral. Once this first pass at the data was complete, we returned to the recordings a second time and transcribed each video. 
Following the transcription phase of the analysis, the videos were each watched a third time, with transcriptions in hand. The interviews were watched here to both ensure transcriptions were accurate and to ensure no potentially beneficial information was left unnoticed during the original timestamping. We identified how students were able to progress through the preestablished layers of the RIF, if at all. We also looked to identify any time a student expressed a connection between a component of the definite integral and the problem being worked on. This analysis allowed us to slightly refine the research questions that our study was able to answer. As themes began to grow from the data, we categorized these themes to draw connections across interviews. Here, we started to see how this group of students assigned meaning to the individual components of the definite integral and how this symbolic meaning metamorphized as the interviews progressed. Ultimately, we were able to define themes that fell into one of three categories: how students interpret the integrand, how students interpret the differential, and how students interpret their interaction.

\section{Results}

While progressing through the two questions in the interviews, it became very apparent to us that there were a variety of ways students interpreted the integrand $(f(x))$, the differential $(d x)$ and their interaction. These elicited subsets of the students' concept images were greatly impacted by the situation in which the definite integral was required. In these results we will discuss how students perceived and understood the integrand in the context of an area under the curve problem, an accumulation of rates problem, and in general as a component of the definite integral. The same will be done for the interaction of the integrand and the differential in all three contexts and how it relates to the product layer in the RIF. Finally, we will discuss how students interpreted the differential. 
In these responses it is important to note that a student's elicited concept image was not static. As will be evident below, students frequently expanded, changed, and even contradicted their understanding of these definite integral components as the interview progressed. Because of the dynamic nature of students' understanding, careful attention will be brought to when these outlooks on the definite integral were a student's first intuition and when they were a newer revelation. We are not distinguishing between newly acquired and newly elicited concept images but simply when understanding is not initially evident to the researchers. To help guide the discussion below, we will first provide a narrative of the progression Zoey demonstrated throughout the interview process. We will then dive deeper into a synthesized analysis across interviews to gain a better understanding of how these students viewed the definite integral.

\section{The Case of Zoey}

Of all the interviewees, Zoey not only demonstrated the greatest ability to complete the varying layers of RIF, but she was able to do so in a way that demonstrated vast improvement while progressing through the area under a curve and application context problems.

Beginning with question one, when initially asked to find the exact area under the given

curve, Zoey demonstrated very little hesitancy in writing $\int_{1}^{7} f(x) d x$ as her answer. Just like many of her peers, the initial rationalization for this integral went no further than an AUC concept image. Understandably, when asked to find the area under a curve, the connection to a definite integral is relatively easy to memorize. Hesitancy began when asked to explain why this area under a curve and definite integral connection existed. Zoey's first inclination was to exclaim that, "the integral is the opposite of the derivative, I know that." While shifting uncomfortably and searching for a rationale, she was unable at this time to provide any explanation, supporting the notion that she initially expressed an AUC concept image. 
Discussion then progressed to providing any graphical meaning behind the individual symbols that comprised the definite integral. This was our first attempt at guided reinvention to provide deeper meaning, more closely paralleling the definition of the definite integral. After quickly explaining how the one and seven designated the $x$ bounds of integration, Zoey stated that the integral symbol in combination with the differential told us that an integral was being taken.

Initially to Zoey, the $f(x)$ represented the function as a whole rather than the height of the function at any $x$ value. This interpretation of the integrand viewed $f(x)$ as a static object, rather than a set of infinite values. At this point, the integral symbol and the differential had no graphical representation for Zoey. When specifically asked about the differential and what meaning it contributed, Zoey said, “I know I messed up a couple times this semester when I didn't put $[d x]$ there, so I think you need it to actually take the integral." She understood the $d x$ was needed in the integral but understanding of it did not go beyond simple recall at this point in the interview. Once the initial discussion of each symbol was complete, Zoey believed that multiplication occurred between the integrand and differential. We will see soon, however, Zoey's uncertainty in this claim. This concluded the initial discussion of how Zoey understood how to calculate the exact area under the curve.

Questions now shifted to how an approximation would be achieved for this area. Zoey quickly created a table with $x$ and $f(x)$ values for each integer one to seven. Unprompted, Zoey was able to take the values from the table she created and correctly perform the calculations necessary for a right-hand sum approximation. When asked to represent her right-hand side approximation graphically, Zoey also was able to draw rectangles on her graph resembling a typical Riemann sum approximation with relative ease. While shading in her first rectangle, Zoey said, "so if we did the right-hand sum, we're taking this and saying that 25 is what's between one 
and two and then all the way down. So, it would be filling that [area] in”. In saying this, Zoey drew a right-hand rectangle from $x=1$ to $x=2$ and shaded in a rectangle with a height of 25 to represent the area. This process of shading was repeated for the remaining rectangles, as seen in Figure $7 \mathrm{a}$.

Zoey was then asked to revisit the symbols that comprised the definite integral to see if any added understanding could be extracted now that we had an alternative way to look at the area under the curve. As originally stated, Zoey still believed $f(x)$ represented the entire curve (Figure 7a). But now, when asked how to interpret the integrand in terms of the rectangles, $f(x)$ represented the top of each rectangle (Figure $7 \mathrm{~b}$ ). Another leap in visually representing the integrand occurred when Zoey began attribute graphical meaning to her right-hand side approximation. Zoey understood the one in her calculations represented the width of the rectangles. Here, Zoey was able to rationalize the integrand did not represent just the top of the rectangles, but the height of the rectangle as well (Figure 7c). She summarized her conclusion by saying, "it gives me my length and my width. So, 58 represents the entire $y$ value covered and then one represents the entire $x$ value covered and then when you multiply them, you get the area of the entire rectangle".

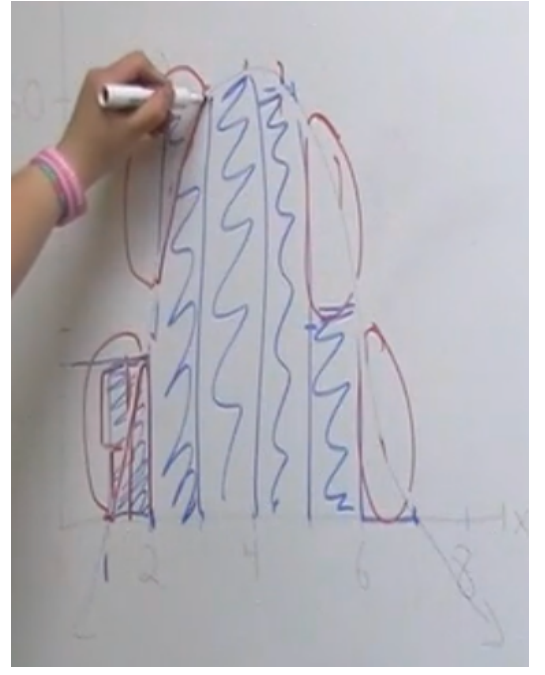

Figure 7a: Zoey demonstrating $f(x)$ represents the curve

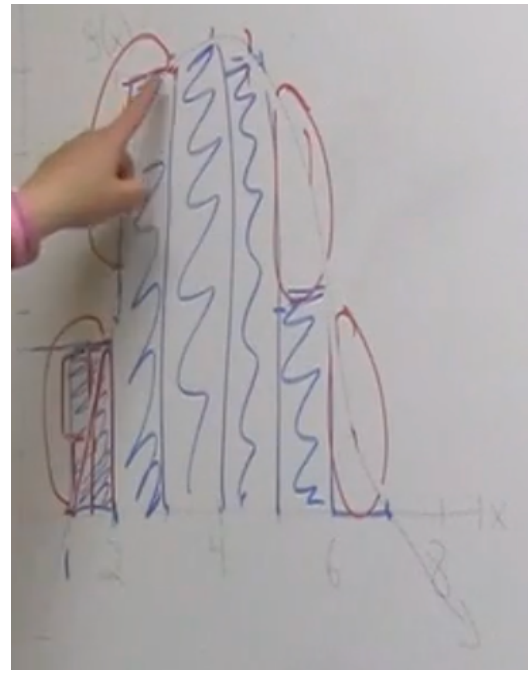

Figure 7b: Zoey demonstrating $f(x)$ represents the top of the rectangle

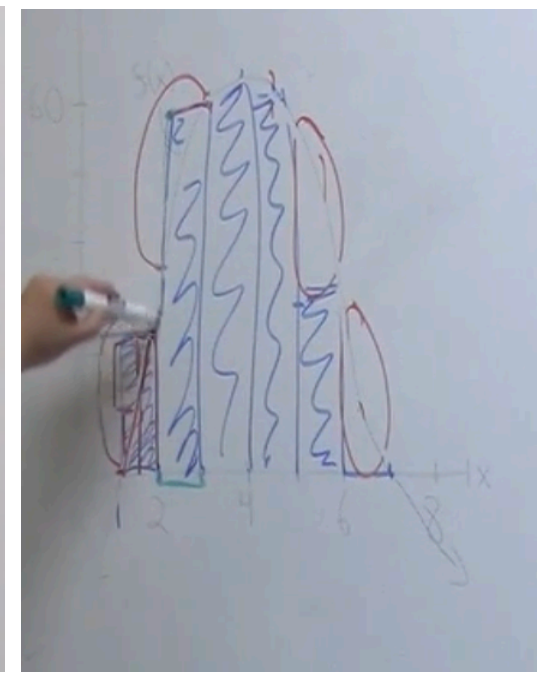

Figure 7c: Zoey demonstrating $f(x)$ represents the height of the 
Zoey was able to draw connections between the widths of her rectangles and the $\Delta x$ in her approximation computation, and yet still felt confident that no graphical meaning for $d x$ existed in her concept image. Originally, Zoey had stated there was multiplication occurring between the $d x$ and $f(x)$ in the definite integral. In the context of the rectangles, Zoey also believed the widths and heights were being multiplied. The connection between these two ideas, however, was not made until much later in the interview. In both representations the $f(x)$ and height of the rectangles were now analogous in her mind. But at this time, switched away from believing multiplication was occurring between $f(x)$ and $d x$. Now, Zoey instead believed information about the width was encapsulated in the integral symbol and its bounds. When using the interval from two to three to describe her understanding, Zoey stated, "because the two to three is still the one to seven in the equation I think". Here, Zoey is expressing the idea that when breaking up the interval from one to seven into six rectangles, she is essentially breaking the integral symbol into sections one to two, two to three, and so on. The rectangle from two to three is approximating $\int_{2}^{3} f(x) d x$.

At this point, we moved onto question two, the application problem, which required an integral to solve. Zoey was given a rate at which the fire spread, and was tasked with calculating the total amount of house engulfed by the flames at time $a$. Initially, Zoey did not see the necessity for the integral. Instead, she simply plugged $a$ into the given rate equation. But by looking at the units, Zoey saw that the given units were $\frac{f t^{2}}{\min }$ and the desired units were $f t^{2}$ so we needed to get rid of the minutes.

Cody: What should our units look like?

Zoey: So, we are looking at the amount of the house engulfed, so we want feet squared Cody: So, we want feet squared. What are the units of this?

Zoey: That's the rate in square feet per minute, so that's square feet per minute. So, we want to take the minute out because we just want square feet. So, this is going to give me a rate because this is in feet squared per minute. So now I would take the integral of this to get the amount. So, I would take the integral of the equation and 
then that would end up giving me the amount. So that would end up going to feet squared.

At this point, Zoey demonstrated a recall concept image for the definite integral based on a rate and amount relationship she learned in class. Just like many of the other students interviewed, Zoey knew through memorization that to get from a rate to an amount, an integral was required. Rote memorization was apparent because initially, Zoey drew her pictorial reference backwards, stating that to get from a rate to an amount, a derivative was required. Once done correcting her mistake, Zoey immediately modified her work and understood an integral was used to answer the problem. In this integral, Zoey wrote her differential as $d x$ instead of $d t$, adding to the evidence that little meaning was being attributed to the differential.

Just as with question one, Zoey was now tasked with providing a rationalization for the necessity of an integral that would push her beyond the two lesser concept images of AUC and FTC. Zoey's first goal was to explain how the integral transformed the units from $\frac{f t^{2}}{\min }$ to $f t^{2}$. When asked by the interviewer, "so what would you have to do to that mathematically, so just the units, what would you have to do to $\frac{f t^{2}}{\min }$ to get $f t^{2}$ ?", Zoey was quickly able to announce, "multiply by minutes". At the very beginning of the interview, Zoey had believed multiplication occurred between $f(x)$ and $d x$. She then changed her mind, stating that the integral symbol is what represented the rectangle widths. At this point in the interview, Zoey stayed consistent with her recent claim by saying, "if we go back to like the last problem, [the integral symbol] is going to give me my $x$-value and [the function] is going to give me my $y$-value." After stating this, Zoey had about two minutes of relatively silent reflection, attempting to confirm this previous statement. 
Zoey then had her epiphany moment by exclaiming, "all [the integral symbol] means is that those are your bounds. So, if you multiplied $[f(x)]$ by $d x$, I guess that this could be the $y$-value and this could be the $x$-value. Distance of $x$ ! Is that what it means?" She quickly switched the multiplication back to the integrand and differential and repositioned the minute units to align with her $d x$, as shown in Figure 8. Forty-five minutes into the interview, Zoey was now able to apply substantive meaning to the integrand, the differential, and their interaction. Excited about this new revelation, Zoey revisited question one to apply the connections she had just developed.
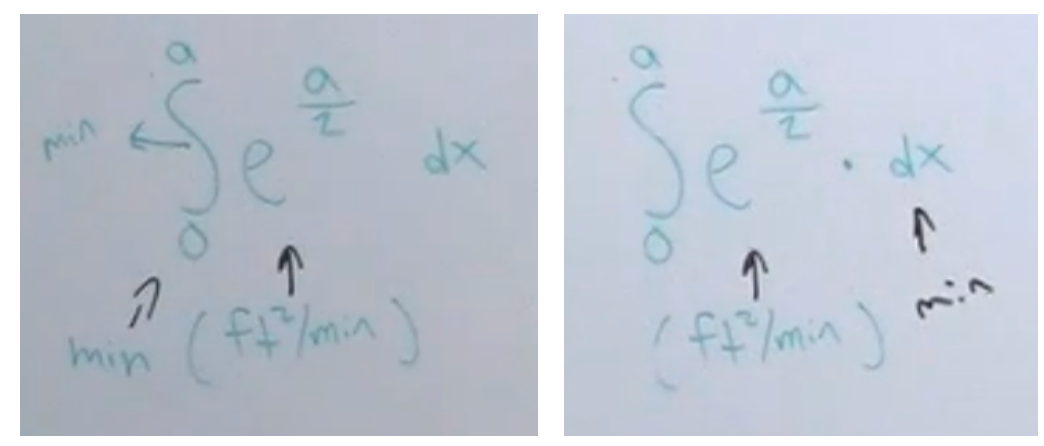

Figure 8: Different ways Zoey attributed units to the definite integral

In her summary, the one and seven still represented her $x$ bounds. Confidently, she indicated that $f(x)$ represented the height of each rectangle and $d x$ represented the width. These two multiply to give the area of each rectangle. Zoey also felt strongly that an analogous multiplication was occurring between the function and $d x$. This distinction is important to note because, as we will see, many students quickly declared multiplication was occurring graphically to represent area, but very few students were able to make the connection that this multiplication could be represented in the definite integral as well. The integral symbol had the meaning of adding all the rectangles up to obtain an overall approximation. Even though her example used only six rectangles for an approximation, Zoey was able to understand that the integral was asking for an infinite number of rectangles to be used. This bridged the final gap between the approximations 
calculated by a finite number of Riemann sum rectangles, and the exact area under a curve the definite integral provides.

While all students interviewed were not able to demonstrate the same level of progression through this guided reinvention, many positive strides at eliciting understanding resembling a Riemann sum definition of the definite integral occurred. Zoey's case study will be used to guide further discussion to help better understand how students were able to rationalize the necessity of a definite integral when descriptions reflecting an AUC or FTC concept image were not sufficient.

\section{Understanding the Integrand}

We will begin by discussing how students interpreted the integrand in the context of an area under the curve problem. Below is Table 2, summarizing the varying levels of understanding students expressed throughout the interviews. If a cell is filled with $\mathrm{Y}(\mathrm{O})$, this indicated that the given student expressed that level of understanding as an original response, whether correct or not. As an example, Zoey originally interpreted $f(x)$ as statically representing the curve as a whole unit. If a cell is filled with $\mathrm{Y}(\mathrm{NR})$, this means a student presented this level of understanding at some point throughout the interview, but this expression was a new revelation, and not naturally elicited. For example, through guided reconstruction, Zoey later described the integrand as representing the height of each rectangle.

Table 2: Ways Students Interpreted $f(x)$

\begin{tabular}{|l|l|l|l|l|}
\hline & $\begin{array}{c}f(x) \text { is static } \\
\text { curve }\end{array}$ & $f(x)$ is height & $\begin{array}{c}f(x) \text { is length } \\
\text { and width }\end{array}$ & $f(x)$ is area \\
\hline Zoey & $\mathrm{Y}(\mathrm{O})$ & $\mathrm{Y}(\mathrm{NR})$ & & \\
\hline Arthur & & $\mathrm{Y}(\mathrm{NR})$ & & $\mathrm{Y}(\mathrm{O})$ \\
\hline Chase & $\mathrm{Y}(\mathrm{O})$ & $\mathrm{Y}(\mathrm{NR})$ & $\mathrm{Y}(\mathrm{NR})$ & \\
\hline Jamie & $\mathrm{Y}(\mathrm{O})$ & & & \\
\hline Jeremy & & $\mathrm{Y}(\mathrm{NR})$ & & $\mathrm{Y}(\mathrm{O})$ \\
\hline Laura & $\mathrm{Y}(\mathrm{O})$ & $\mathrm{Y}(\mathrm{NR})$ & & \\
\hline Oliver & & $\mathrm{Y}(\mathrm{O})$ & $\mathrm{Y}(\mathrm{NR})$ & $\mathrm{Y}(\mathrm{NR})$ \\
\hline
\end{tabular}


Upon asking the students how to calculate the exact area under the given curve, all of the students but Oliver were able to produce some form of a definite integral, most mirroring $\int_{1}^{7} f(x) d x$. Table 3 summarizes the integral each student initially wrote and what the definite integral ended up looking like. Since Oliver was not able to produce an integral on his own, his information is not included in the table. Even though he did not create the integral on his own, when presented with a definite integral and asked how this expression related to the graph, Oliver quickly said, "That's the area of the whole thing." While saying this, Oliver was motioning to the area under the curve. Just like Zoey, all of the students interviewed possessed a strong connection between the definite integral and calculating the area under a curve. By simply stating a definite integral was the correct tool to determine the area under the curve, it was not yet evident which students possessed a level of understanding beyond an AUC concept image.

Table 3: Ways Students Wrote the Definite Integral

\begin{tabular}{|l|c|c|}
\hline Zoey & $\int_{1}^{7} f(x) d x$ & $\int_{1}^{7} f(x) d x$ \\
\hline Arthur & $\int_{1}^{7} x^{3}-18 x^{2}+87 x-70 d x$ & $\int_{1}^{7} f(x) d x$ \\
\hline Chase & $\int_{1}^{7} f(x) d x$ & $\int_{1}^{7} f(x) d x$ \\
\hline Jamie & $\int_{1}^{7} f(x)$ & $\int_{1}^{7} f(x)$ \\
\hline Jeremy & $\int_{1}^{7} f(x)-0 d x$ & $\int_{1}^{7} f(x) d x$ \\
\hline Laura & $\int_{1}^{7} x^{3}-18 x^{2}+87 x-70-0 d x$ & $\int_{1}^{7} x^{3}-18 x^{2}+87 x-70 d x$ \\
\hline
\end{tabular}


These questions about calculating the area under the curve were then followed by us asking the interviewees how, if at all, does the integrand component of the definite integral relate to the picture they had been interacting with up to this point in the interview. Surprisingly, even though immediately before this question we had been discussing area under the curve, one of the most common graphical connections the students made were to the curve itself, not the area. Just as Zoey had explained, Chase, Laura, and Jamie all initially stated that $f(x)$ represented the curve or an upper bound of the function.

Cody: So, the $f(x)$, what does it mean in terms of our picture?

Chase: It's telling you the curve right here.

This indicated to us that when discussing the area under the curve, students frequently viewed the function as a static object/boundary rather than an infinite set of inputs and outputs. Jones (2013) also saw this connection in student concept images between $f(x)$ and a static upper bound for a region. This data strengthens this notion by demonstrating that even when students are initially prompted to be in the area under the curve frame of mind, they still often lacked the instincts to think of $f(x)$ as anything more than a curve, hovering some distance away from the $x$ axis.

Encouragingly, this interpretation of the integrand changed when approximating the area with a Riemann sum. At some point in the interviews, all of the students interviewed had successfully created a visual representation that allowed for the approximation of the area under the curve via vertical rectangles (Hood \& Sealey, in preparation). With the aid of a visual approximation analogous to a typical Riemann sum approximation, Chase, Arthur, Jeremy, Oliver, and Laura joined Zoey in interpreting $f(x)$ as the height of the rectangles. This was a new graphical interpretation of $f(x)$ for all of these students. Chase stated, "now that we're looking at it in terms of rectangles, I think that $f(x)$ actually refers to the height." The difference between these two 
interpretations is in the first case, students viewed $f(x)$ as an identifier for the equation provided. With this second representation however, students' elicited concept image of $f(x)$ now included a geometric component that potentially could provide additional meaning to the integrand. Jamie was the only student who never provided evidence that she thought of $f(x)$ as a height during her interview. Even when incorporating rectangles into her mental image, she only described $f(x)$ as the "top" or "max" of her rectangles.

Interestingly, Oliver was the only student to overlook the original question of calculating the area exactly. When asked this question at the beginning of the interview, he immediately began breaking the region into rectangles that eventually would align with a traditional graphical representation of a Riemann sum. Oliver was also the only student who initially described $f(x)$ as a height. Just like many other students, Oliver later altered his graphical representation of $f(x)$. Nevertheless, it is interesting that the students who initially interpreted the area under the curve as a definite integral also were more likely to naturally describe $f(x)$ as an identifier. Whereas Oliver began discussing the area under the curve as a Riemann sum and he also thought of $f(x)$ initially as a height.

While thinking of $f(x)$ as either an identifier for a curve or as a height were the two most common ways students expressed their concept images of the integrand, there were many other descriptions as well. Arthur initially thought $f(x)$ represented, "area or just like everything under it." Jeremy came to a similar conclusion when first asked this question, but quickly changed his answer, aligning more with students who interpreted the integrand as a curve identifier, " $f(x)$ is just everything under this curve, well $f(x)$ represents the curve. Everything underneath of it is going to be your area." Even Oliver later in his interview thought the integrand alone represented the entire area under the curve. As we will later see, this potential confusion for having $f(x)$ 
overrepresent the entire area rather than just one component of the area calculation could easily be attributed to student hesitancy in understanding $d x$.

Oliver overattributed meaning to $f(x)$ at a different point in the interview as well when he claimed it represented both the height and the width of each Riemann sum rectangle.

Cody: In terms of your rectangles, do you see $f(x)$ contributing any information to your rectangles at all?

Oliver: Yeah, the length times width for each [rectangle] represent my $f(x)$.

Chase was the only student to initially see $f$ and $x$ as two separate components being multiplied. This caused him to draw a similar conclusion to Arthur, Jeremy, and Oliver that $f(x)$ (or in his case $f$ times $x$ ) alone represented the area under the curve. Once actual numbers were incorporated into his approximation using a Riemann sum, Chase decided the number corresponding to height came from the $f$ and the number corresponding to width came from the $x$ in $f(x)$. It is clear that at this point in the interview Chase viewed the integrand itself as a product, rather than a function with a single variable input.

\section{Understanding the Product of the Integrand and Differential}

For this collection of students, little to no connections between the differential and how it related graphically to a Riemann sum occurred until after understanding of the product layer was brought to the forefront of the students' minds. Because of this progression in concept image evocation, we will first discuss how students expressed understanding of the product between $f(x)$ and $d x$. Student understanding of the relationship between these two components of the definite integral will be discussed through the lens of Riemann sum rectangles, the definite integral notation, and unit manipulation required to convert a rate to an accumulation via a definite integral. This discussion will be followed by a description of student understanding of the differential in 
isolation. As with student understanding of the integrand, Table 4 summarizes how students' concept images reflected this product relationship.

Table 4: Ways Students Interpret the Product Layer

\begin{tabular}{|l|l|l|l|l|l|l|}
\hline & $\begin{array}{c}\text { Multiplication in } \\
\text { rectangles }\end{array}$ & $\begin{array}{c}\text { Multiplication exists } \\
\text { in integral }\end{array}$ & $\begin{array}{c}\text { Multiplication } \\
\text { between } f \text { and }(x)\end{array}$ & $\begin{array}{c}\text { Multiplication } \\
\text { between integrand } \\
\text { and integral symbol }\end{array}$ & $\begin{array}{c}\text { Multiplication } \\
\text { between } f(x) \text { and } d x\end{array}$ & $\begin{array}{c}\text { Multiplication in } \\
\text { units for problem 2 }\end{array}$ \\
\hline Zoey & $\mathrm{Y}(\mathrm{O})$ & $\mathrm{Y}(\mathrm{O})$ & $\mathrm{Y}(\mathrm{NR})$ & $\mathrm{Y}(\mathrm{O}$ and NR) & Y (NR) \\
\hline Arthur & $\mathrm{Y}(\mathrm{O})$ & $\mathrm{Y}(\mathrm{NR})$ & & & $\mathrm{Y}(\mathrm{NR})$ \\
\hline Chase & $\mathrm{Y}(\mathrm{O})$ & $\mathrm{Y}(\mathrm{NR})$ & $\mathrm{Y}(\mathrm{NR})$ & & $\mathrm{Y}(\mathrm{NR})$ & Y (NR) \\
\hline Jamie & $\mathrm{Y}(\mathrm{O})$ & $\mathrm{Y}(\mathrm{NR})$ & & & & $\mathrm{Y}(\mathrm{NR})$ \\
\hline Jeremy & $\mathrm{Y}(\mathrm{O})$ & $\mathrm{Y}(\mathrm{NR})$ & & & $\mathrm{Y}(\mathrm{NR})$ & $\mathrm{Y}(\mathrm{NR})$ \\
\hline Laura & & $\mathrm{Y}(\mathrm{O})$ & & $\mathrm{Y}(\mathrm{O})$ & & \\
\hline Oliver & $\mathrm{Y}(\mathrm{O})$ & $\mathrm{Y}(\mathrm{O})$ & $\mathrm{Y}(\mathrm{O})$ & & & \\
\hline
\end{tabular}

Of the varying ways a student could understand the product layer, graphically with the aid of Riemann sum rectangles provided students with the least difficulty. At some point in the interview process, all students but Laura were able to identify that multiplication occurred between length and width to determine the area of the rectangles used for approximation. This gives credence to the idea that students have a great ability to understand how to approximate the area under the curve using the aid of Riemann sum rectangles. When asked how the units change as a result of the integral for question two, Laura confusingly stated, "it like cancels out somehow and I can't figure it out." Her inability to justify the transformation of units could easily be attributed to the gap formed between her approximations using a finite number of rectangles and her exact calculation using an infinite number of vertical lines with no width. When Laura was asked how the integral resulted in the exact area under a curve, her description utilized lines with no width, instead of rectangles. This prevented Laura from visually seeing any multiplication of width and height in her justification. In turn, it would be presumptive to suggest Laura would then be able to see the necessity of multiplication and unit manipulation in the definite integral itself.

While many students felt confident identifying multiplication while calculating the area of rectangles, this comfortability was not reflected when discussing $\int_{1}^{7} f(x) d x$ notation. Zoey was 
actually the only student to naturally assume multiplication occurred between $f(x)$ and $d x$. And even so, as previously described, still showed great hesitancy with this claim. Arthur and Jamie both believed that even though multiplication occurred in the rectangular approximation, there was no multiplication occurring within the symbols that comprise the definite integral. Chase originally had the same inclination, but as the interview progressed, changed his response. Oliver and Laura believed there was multiplication within the definite integral, but both weren't necessarily certain where the multiplication occurred. At one point, both Chase and Oliver said the multiplication was self-contained in the integrand. While this misconception wasn't pursued further, this error could have been a result of the previously discussed misinterpretation of the notation $f(x)$ as $f * x$. As discussed previously, at one point in her interview, Zoey believed the multiplication occurred between the integral symbol and $f(x)$. Laura supported this idea of where the multiplication was present. Laura's assumption comes less surprising because her concept image seldomly included the $d x$, a result of envisioning an infinite number of lines, rather than rectangles.

During question one, there was little consensus on where a product, if any, was occurring in the definite integral. Fortunately, question two and its reliance on units was able to bring to light this product layer for many students. Initially, only Chase saw the need of an integral to transform a given rate to an accumulation, a requirement to gain the correct solution. For most of these students, once focus was placed on the units of the given rate $\left(\frac{f t^{2}}{\min }\right)$ and the units of the required outcome $\left(f t^{2}\right)$, connections began to occur. At some point in the interview, every single student was able to rationalize on his or her own that a definite integral was required to achieve the total area of house engulfed by flames. Almost every student also referenced the relationship they remembered from class between rate and accumulation as a tool to recognize the necessity of an integral. As an example, when asked why an integral was required to solve this problem, Jamie 
explained it was, “because it goes from squared feet per minute and my question's asking for an amount, so I have to do the integral.” As shown in Figure 9, Arthur, Chase, Jamie, Jeremy, Oliver, and Zoey were able to pictorially represent this relationship between an amount and a rate. All of these students had seen a similar visual aid in class at some point as well.
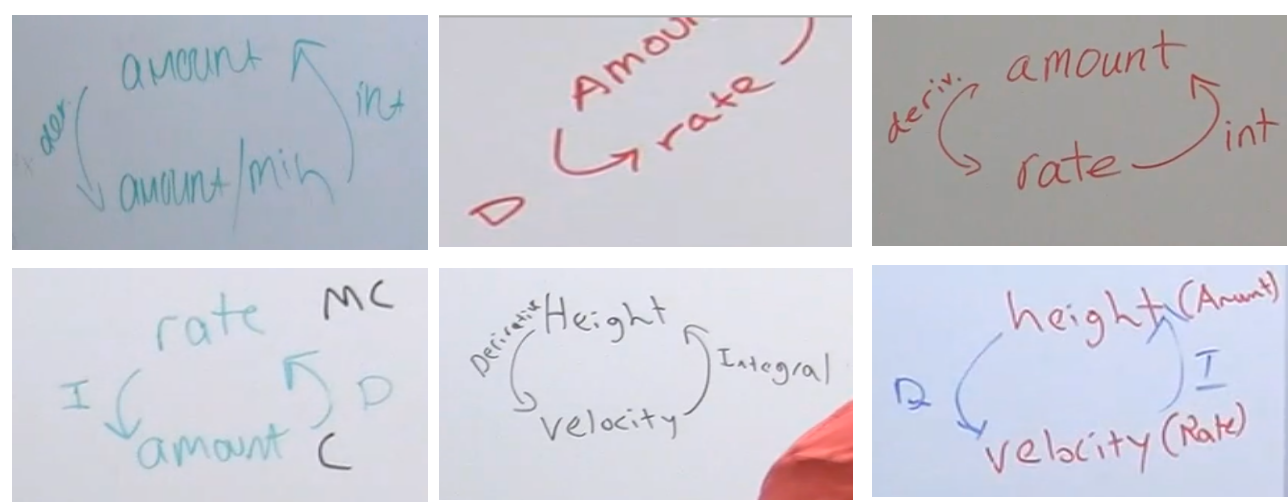

Figure 9: Students demonstrating relationship between rates and amounts

While this reliance on recall aided many students in understanding a definite integral was required to answer the question, we wanted to see if the students' concept images could go beyond rote memorization of the FTC. Discussion of the units played a key role in extracting this knowledge. Zoey knew that multiplication between $\frac{f t^{2}}{\min }$ and $\min$ must occur to obtain the desired units. Arthur, Chase, Jamie, and Jeremy were able to draw a similar conclusion. This was the first time that many of these students mentioned the idea that multiplication occurred in the context of the definite integral notation. Laura and Oliver both knew the given units and the desired units were bridged by an integral, but neither were able to attribute this connection to multiplication.

Chase and Jeremy joined Zoey in showing great progress in extracting a Riemann sum concept image through this guided reconstruction with the aid of a real-life example. These three students were all eventually able attribute the minute units to the differential and exclaimed that the previously assumed multiplication occurred between the integrand and the differential (Figure 10). Unfortunately, Jamie, Oliver and Arthur understood that the function was being multiplied 
somehow by $\min$, but were never able to actually attribute this multiplication to any component of the integral. Jamie simply concluded that, "the calculator works its magic" to bridge this gap.

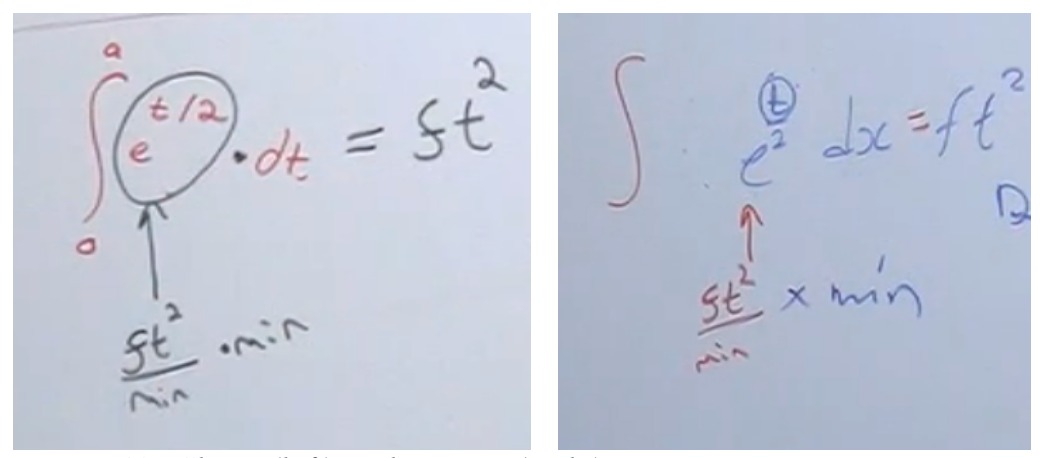

Figure 10: Chase (left) and Jeremy (right) assigning units to

Cody: You said the minutes are going to basically go away, what can we do to this to make the minutes part go away? If we have feet squared per minute and we want to turn it into eventually feet squared, what would we have to do to this to get feet squared eventually?

Chase Cancel the minutes out.

Cody: And how would we cancel it?

Chase You'd have to multiply by minutes in this case.

Cody: Okay and then what would happen then?

Chase: The minutes would cancel.

Cody: Okay so that's kind of the thing we're looking for right now. Do you see anyway that you could rationalize "oh this kind of represents the minutes so that maybe be able to cancel it out" or not?

Chase: I think in this case it's where that dx comes in maybe?

Cody: Okay so explain that to me because this is really the first time we've talked about the $d x$.

Chase: Yeah, I don't know I'm just thinking because I know that this would be square feet over minutes and this is the only thing its being multiplied by... Sure they're being multiplied let's go with that.

This conclusion of multiplication was not discussed by either Chase nor Jeremy prior to the use of units and an application context. Even though all of the interviewees were not able to completely progress through the product layer, the use of both rectangular approximations and a real-life example helped improve understanding for every student being interviewed. 


\section{Understanding the Differential}

Even amongst experts, there is very little agreement on how to interpret $d x$ in the context of definite integrals, indefinite integrals, and differential equations (McCarty \& Sealey; 2019). McCarty \& Sealey found that some experts are hesitant to describe $d x$ as having size, while others describe it as a very small piece of $x$. Some believe it to be a real number, while others say it is infinitesimal in size. Some say its size does not correspond to a number and is simply "small", while others utilize it as a symbolic tool for differentiation and integration. In this section of the results, we are not ascertaining that perceiving the differential as an infinitesimal width is necessarily accurate or a requirement. We simply are adding to the list of roles a differential can play. In the context of relating definite integrals to a Riemann sum, we believe the differential can be connected to the limiting nature of delta $x$ to enhance meaning.

Discussion of the differential in a graphical and application context provided a much wider variety of elucidations than the integrand did. We will see that recent work (Doughty et al., 2014; Sealey \& Thompson, 2016) describing student difficulty with understanding $d x$ was confirmed by these interviews as well, resulting in a great variety of responses. In Table 5, we summarize the ways students interpreted the differential.

Table 5: Ways Students Interpret the Differential

\begin{tabular}{|l|l|l|l|l|l|l|}
\hline & $\begin{array}{c}d x \text { has no known } \\
\text { meaning }\end{array}$ & $\begin{array}{c}d x \text { is required in } \\
\text { integral }\end{array}$ & $d x$ has units & $\begin{array}{c}d x \text { corresponds to } \\
\text { width of rectangles }\end{array}$ & $\begin{array}{c}d x \text { relates to } \\
\text { derivative }\end{array}$ & $\begin{array}{c}d x \text { is variable of } \\
\text { integration }\end{array}$ \\
\hline Zoey & $\mathrm{Y}(\mathrm{O})$ & $\mathrm{Y}(\mathrm{O})$ & $\mathrm{Y}(\mathrm{NR})$ & $\mathrm{Y}(\mathrm{NR})$ & $\mathrm{Y}(\mathrm{O})$ & \\
\hline Arthur & $\mathrm{Y}(\mathrm{O})$ & $\mathrm{Y}(\mathrm{O})$ & & & & \\
\hline Chase & $\mathrm{Y}(\mathrm{O})$ & $\mathrm{Y}(\mathrm{O})$ & $\mathrm{Y}(\mathrm{NR})$ & $\mathrm{Y}(\mathrm{NR})$ & $\mathrm{Y}(\mathrm{O})$ & \\
\hline Jamie & $\mathrm{Y}(\mathrm{O})$ & & & & & \\
\hline Jeremy & $\mathrm{Y}(\mathrm{O})$ & $\mathrm{Y}(\mathrm{O})$ & $\mathrm{Y}(\mathrm{NR})$ & $\mathrm{Y}(\mathrm{NR})$ & $\mathrm{Y}(\mathrm{O})$ & \\
\hline Laura & $\mathrm{Y}(\mathrm{O})$ & & & & $\mathrm{Y}(\mathrm{O})$ & \\
\hline Oliver & & & & & $\mathrm{Y}(\mathrm{NR})$ & $\mathrm{Y}(\mathrm{O})$ \\
\hline
\end{tabular}

In the beginning of each interview, once the definite integral was first produced, we asked the students what meaning each component held. This question often was presented after the student established that a definite integral would be used to calculate the area under the curve. 
Initially, every single student except for Oliver had the initial response that $d x$ has no meaning. Even so, Oliver said initially $d x$ 's only role was to describe what independent variable the function would be using. Even though $d x$ lacked so much meaning for the students, Arthur, Chase, and Jeremy all agreed with Zoey that it was required. Arthur explicitly said early in the interview. "I don't know what $d x$ means, I just put it there." In the context of problem two, when asked why he included the $d x$ in his notation, Chase responded with, "because it's part of the integral, I'm not sure." Both of these examples demonstrated that many students may include the differential in their integral notation through conditioning, without really gaining any added value by including it. Jamie was the only interviewee to initially produce a definite integral that did not include $d x$.

Students began attributing meaning to the differential when asked to relate each symbol that comprised the definite integral to the newly formed vertical rectangles, that when summed, would approximate the area under the curve. In this instance, students were, for the first time, prompted to draw connections between the integral, a Riemann sum approximation, and area under the curve beyond ones that resemble nothing more than an AUC or FTC concept image.

At this point in the interviews, every student except for Laura had successfully produced both a definite integral as a way to calculate the area under the curve exactly, as well as traditional Riemann rectangles that would be used to computationally approximate the same area. When asked what graphical meaning the $d x$ had, if any, many students showed hesitancy in answering the question. It was almost as if they wanted to provide a constructive response but did not naturally have a graphical representation of the $d x$ in their concept image. Some of the resulting connections never managed to relate back to the graph. Jamie and Chase thought the $d x$ alluded to $u$ substitution, a technique used to calculate an antiderivative. In a similar context, Laura, Chase, Jeremy, Zoey, and Oliver hypothesized that the $d x$ in the definite integral stood for the derivative. 
When asked what the $d x$ represented, Laura said, "I always thought that meant like the derivative of what you're trying to find."

Initially, Chase, Arthur, Jamie, and Jeremy agreed with Zoey that $d x$ had no graphical meaning. For Chase, the integral symbol, rather than the differential, provided instructions to break the $x$-axis into widths that would later be used to construct rectangles. While no students were able to attribute any substantial graphical meaning to the $d x$ early in the interview, Zoey still felt there was a connection she was simply unaware of, "I'm curious if the $d x$ means anything."

While many students began by attributing only nongraphical meaning to $d x$, progress began to occur when question two was introduced. As a reminder, once sufficient time was spent understanding how students deconstructed and applied meaning to the symbols that constructed the definite integral in the context of an area problem, they were then given question two. In this second prompt, students were given a scenario where a fire spread through a house at a given rate. This information would be used to determine how much of the house would be engulfed by flames at a given time.

In this problem, $t$ represented the independent variable, time. Even though this would result in the differential being $d t$ instead of $d x$, Jeremy was the only student to actually use $d t$. His rationale, being that since the variable in the function is $t$, the variable in the differential must be $t$ as well. Once it was determined an integral was required to determine total fire spread, Chase, Arthur, and Oliver aligned with Zoey in deliberately using $d x$ instead of $d t$ as their differential. Laura also misidentified the differential but used $d a$. This lack of attention to the differential supports the notion that many of these students lacked a deep and accurate concept image of the definite integral that encapsulated the differential. Even though careful attention was not given to determining the variable in the differential, by understanding the relationship between the 
differential and integrand described in the previous section, Chase, Jeremy, and Zoey were all able to independently assign the units of $\min$ to the differential.

At this point, many interviews returned to question one to summarize and identify any changes in understanding that occurred during question two. As described above in the product layer discussion, great leaps in elicited concept images occurred through the use of the application prompt when heightened attention was given to the units. After hypothesizing the $d x$ had units, both Chase and Jeremy joined Zoey by circling back to the original area under the curve problem and provided new connections between the $d x$ and the graph. Originally, both of these students stated the differential had no graphical meaning. But now, they both said $d x$ represented the widths of the rectangles (Figure 11). Unfortunately, they were not able to progress as far as Zoey had done in extending this approximating nature to an exact calculation using an infinite number of these rectangles.
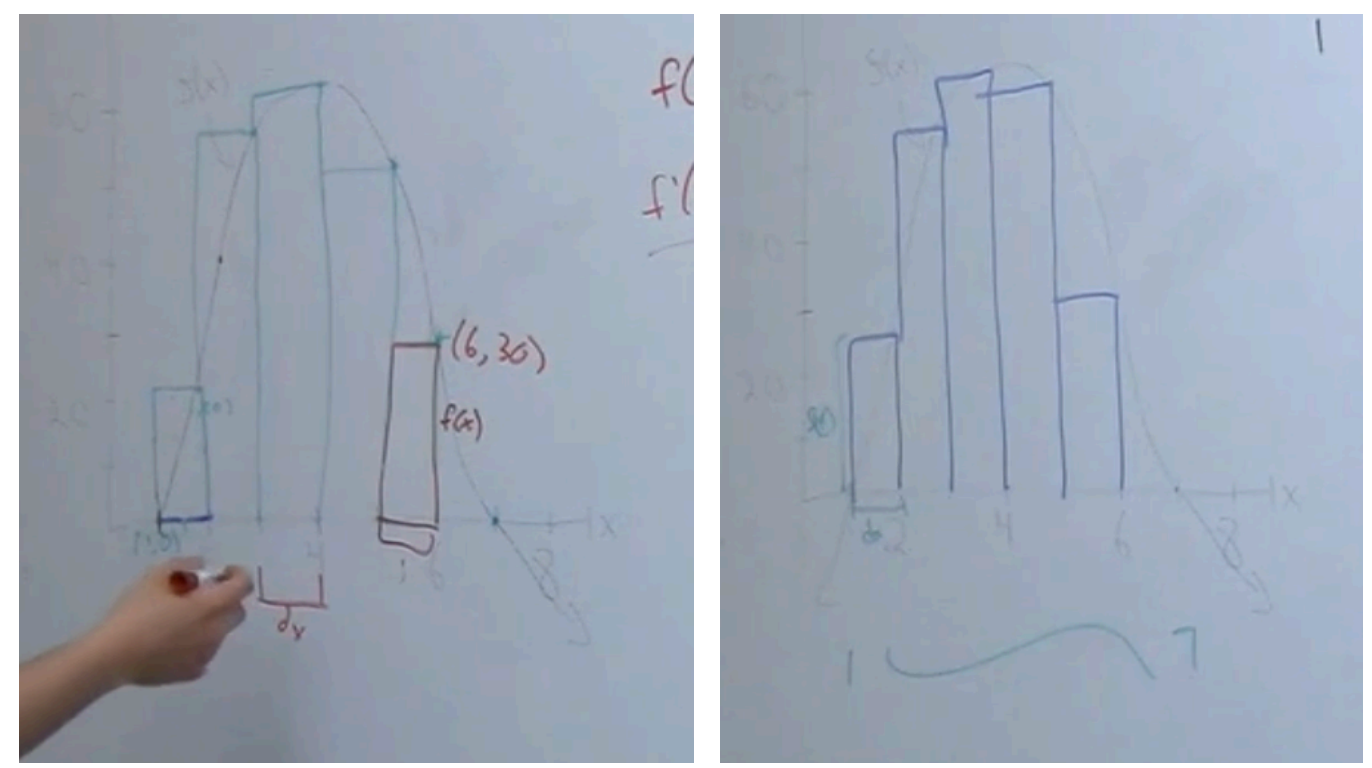

Figure 11: Jeremy (left) and Zoey (right) labeling the widths of the rectangles as $d x$

Even with this dramatic leap in understanding, the infinitesimal nature of the differential still provided Jeremy with concern. He now was able to articulate that the smaller $d x$ became, the better the approximation would be, but no matter how small $d x$ became, this resulting finite width 
would still only be an approximation of the area, never an exact calculation. To Chase and Jeremy, $d x$ represented a finite width, no matter how small. Jeremy described this limitation in his concept image of the differential when saying, "but it's obviously an approximation, you still have this area that's like going to be too much. So, I think maybe the $d x$ is what's reducing the widths so that we can get a more precise answer. Every single time you get smaller and smaller, you're always going to have this curve making an awkward shape that you're not going to be able to get the area of." This, again, attributes much of the misunderstanding of the differential to its infinitesimal nature.

\section{Conclusion}

The definite integral is a complex combination of symbols that many students contextualize as a single entity. Experts strive for students to view this concept as a collection of mathematical ideas that, when combined, provide a much deeper meaning than they do individually (Jones, 2013; Jones, 2015; Meredith \& Marrongelle, 2008; Nguyen \& Rebello, 2011; Rösken \& Rolka, 2007; Sealey, 2014; Von Korff \& Rebello, 2012). By asking students to discover meaning of the integral symbol, integrand, differential, and their interactions, they are being provided with an opportunity to gain information that can move them beyond a simplistic AUC or FTC concept image. This scrutiny provides for a wonderful opportunity to begin a discussion that can help a student's image align more closely to a Riemann sum concept image.

This works supports the work of others, showing that students frequently and naturally express understanding of the definite integral through an AUC or FTC lens (Doughty et al., 2014; Jones, 2015; Nguyen \& Rebello, 2011, Rösken \& Rolka, 2007). We, however, expand upon this to better understand what knowledge is contained in a student's concept image that may resemble understanding more reflective of a Riemann sum concept image. While all of the students relied on recall cues when initially discussing these applications of the definite integral, further 
discussion with the students showed they collectively possessed a much greater and deeper understanding. This harkens back to the notion Jones (2013) work supports that the concept image of a definite integral that a student possesses may vary greatly from the one expressed in a given context.

Of the seven students, Chase, Zoey, and Jeremy were able to successfully demonstrate a level of understanding reflecting the RIF and all its layers. The other four students were able to demonstrate great progress, as well, even though they were unable to produce a complete Riemann sum concept image. All the students were able to demonstrate a strong understanding of the summation layer in the context of adding up rectangles in order to approximate the area under a curve. Zoey, Oliver, Arthur, Chase, Laura, and Jeremy were able to make associations between $f(x)$ and the height of the Riemann rectangles. Zoey, Chase, and Jeremy were able to express connections between the multiplication occurring between $f(x)$ and $d x$ and the multiplication of length and width in the formation of the Riemann rectangles. This ultimately shows a strong understanding by these students of the RIF's product layer. At some point, all the students interviewed were able to make connections reflective of Sealey's (2013) RIF and demonstrate understanding beyond what was initially elicited at the beginning of the interviews.

One important tool these students utilized to grow these connections between the definite integral's symbols and the RIF were the units in a real-life application problem. These units were used as a tool to help draw students to the connection between integrals and multiplication. For Arthur, Chase, Jamie, and Jeremy, the product layer was almost nonexistent in their expressed understanding of the definite integral until it was required to result in the correct area units. Even though these students had memorized the relationship between rates, accumulations, and definite integrals, the transformation of $\frac{f t^{2}}{\min }$ to $f t^{2}$ cued the existence of the min units, somewhere to be 
found. This work demonstrates the importance of units and the role they can play in the development of a student's Riemann sum understanding of the definite integral.

This research provides great development into how students can use the symbolic construction of the definite integral and the units of an application problem to progress through the RIF. Most importantly, however, we have shown the caution that must be used when evaluating a student's level of understanding of a given concept. Not only is it important to provide students with the tools and resources to develop a Riemann sum concept image, it is also crucial to have proper evaluation instruments in place. It is safe to assume that the information a student presents is only the tip of their knowledge iceberg. Greater understanding inevitably exists, just waiting to be extracted.

\section{Teaching Implications}

The AUC concept image utilizes the recall of a fact that statically connects the definite integral to a graphical image. Understandably, this connection is very easy to make and remember in contrast to the complexities involved in fully encapsulating the RIF. This data supports the notion that an instructor should be cautious in drawing conclusions when students discuss the definite integral with no deeper meaning than as an area under a curve. Just because the simplest connection is being vocalized, does not mean that a richer understanding does not exist. For example, when guided to use rectangular partitions of the area, rather than looking at it as one object, a vast majority of the students interviewed made great connections beyond the recall of AUC and extract deep meaning from the integral components.

Many of the misconceptions and reservations these students presented reflected a lack of understanding of the infinitesimal nature of $d x$. We have been able to organize some tools through this study's interview protocol that could potentially ease students into a more accurate 
understanding of the differential. Students should first understand the context of multiplication in the definite integral before having the opportunity to encapsulate meaning behind the differential itself. By understanding that a product is occurring in the definite integral, students are more likely to apply units and graphical meaning to the differential.

Rectangles and realistic applications aid in eliciting information about the components of the definite integral that can be lost when viewing the integral as nothing more than a representation of an exact area under a curve. Rectangles provide a way to remove the infinitesimal aspect of $d x$ by applying a tangible graphical representation for a previously abstract concept. By using realistic applications with familiar units, students have the ability to force meaning onto the differential that may have contained no meaning when discussion was purely computationally based. When students need to find a way to rationalize the change in units an integral creates, many students can do so in a way that elicits both differential and product understanding. By providing instructors and students with the tools this research used to decompose the definite integral, students can be provided the opportunity to develop and discover an understanding of the definite integral that more closely aligns with a Riemann sum concept image.

\section{Future Work}

As this research has demonstrated, it is important to decompose the definite integral into parts and discuss how these parts interact to gain a truer understanding of how students perceive the definite integral. Here, we focus on the integrand, the differential, and their interaction. An entirely new area of information could potentially be opened if emphasis were to be placed on the integral symbol and the boundary components themselves. Gaining knowledge of how students interpret this symbolic construction, potentially in the context of the summation layer could be very insightful. In the interviews discussed above, more than one student referred to the integral 
symbol as an elongated $S$. This description could potentially be explored further to see what, if any, information this $S$ possesses.

We have also been given a glimpse into how the necessity for a change in units elicits the necessity of an integral in many students' minds. Currently, there is very little existing research exploring the idea that assigning units to the integrand and differential can guide a student's understanding that a definite integral is required to resolve a particular application context. We have been able to establish a strong correlation between students who apply units to the differential and those same students who can elicit deep meaning of the differential in the context of Riemann rectangles and the RIF. It may be that the use of units in an application could be a key factor in resolving many of the misconceptions we encountered with the product layer and in turn, the differential. 


\section{SUMMARY}

In this research, I sought to understand how students who were not presented with formal instruction of limits in an Applied Calculus course could demonstrate a Riemann sum concept image level of understanding of the definite integral. In doing so, I placed focus not on what ideas and knowledge students naturally elicited, but what type of understanding could be brought to light through the use of Realistic Mathematics Education and guided reinvention. This guided reinvention occurred in the context of two real-life application scenarios. Little to no actual teaching occurred in these interviews to develop a Riemann sum concept image. Instead, the use of these students' naturally elicited understandings of definite integrals in a variety of real-life contexts resulted in tremendous improvement in demonstrating a Riemann sum concept image understanding.

Students were first asked to calculate the area under a curve. Once it was understood how this area calculation could be achieved, focus was placed on how the visual tool could be used to develop connections between the definite integral, its symbolic composition, and a graph. The second situation requiring a definite integral the students were introduced to involved a fire spreading through a house. The prompt provided an equation representing the rate at which the fire spread, and students were asked to determine how much of the house would be engulfed by flames at time $a$. Once students were able to determine that a definite integral was required to answer this question, focus of the interviews shifted to how this definite integral, the units of the function, and the units of the resulting integral could bridge connections harkening back to the summation, product, and limit layers of Sealey’s (2014) Riemann Integral Framework. While not heavily discussed in either of these papers, it was very evident from these seven interviews that 
students had no difficulty with the summation layer. Instead, the majority of students' time and effort during the interviews was spent understanding the product and limit layers.

In paper one, analysis was done on how students were able to progress through the limit layer of Sealey’s (2014) Riemann Integral Framework. Ultimately, I concluded that this limit layer should be broken into two sublayers within the framework. The purpose of this dissection was to provide a better representation of what aspects of this layer in which students struggled. The first sublayer, approximation sublayer, places focus on improved approximations. Once students in my study were able to develop a Riemann sum approximation for the area under a curve, they showed little difficulty in rationalizing how this approximation could be improved through the use of an increased number of smaller rectangles. With all interview subjects, difficulty arose when this idea of improved approximations shifted to finding the exact area under the curve. This leap in difficulty aligned with the research of others, which concluded students' difficulty with limits often reaches a maximum when connections to infinity are required (Cornu, 1991; Oehrtman, 2008). Once this stage of the improved approximations was entered, I concluded the student had entered the exact sublayer of the limit layer. In Sealey's (2014) development of this framework, students were only asked to make this extension to an exact calculation in a follow-up study. In Sealey's work, students were provided three activities in which only improved approximations were asked of them. This means most of the students in this original study were never asked to extend beyond the newly defined approximation sublayer, which can be summarized by $\lim _{n \rightarrow N} \sum_{i=1}^{n} f\left(x_{i}\right) \Delta x$ for large $N$. In contrast, the students in my study were asked to extrapolate to the exact sublayer, which is achieved when students are able to extend beyond a large number, $N$, to the idea of infinity. This final improvement is needed to obtain the Riemann sum definition of the definite integral, $\lim _{n \rightarrow \infty} \sum_{i=1}^{n} f\left(x_{i}\right) \Delta x$ 
A key tool to eliciting this Riemann sum concept image from students was the use of a graphical aid with which students could actively interact. The physical use of geometric shapes to approximate a definite integral afforded these students with an opportunity to overcome the abstract nature of the symbols described above for the approximation and exact sublayers. The students in these interviews were also able to use this graph to facilitate their explanation for why larger $N$ results in a reduced error. By shading in the varying error corresponding to different types of approximations produced, they were able to convey a strong understanding of the approximation that otherwise may have been left as a thought experiment. While not all of the students were able to extend beyond the approximation sublayer into the exact sublayer, much deeper explanations reflective of these two sublayers occurred once the students began interacting with and writing on the graph.

In paper two, I focused on how meaning could be applied to the symbolic components of the definite integral to overcome a reliance on rote memorization, with the ultimate goal of making connections between these symbols in the definite integral and the layers of the Riemann Integral Framework. Just as with paper one, the prompt of calculating the area under a curve served as a wonderful tool for extracting information contained within the Riemann sum definition of the definite integral. Early in the interviews, a majority of students applied little to no meaning to the differential and did not believe multiplication occurred within the definite integral. But, through the use of Riemann rectangles, these same students were able to attribute graphical meaning to both the integrand and the differential. By the end of the individual interviews, six of the seven students were able to associate $f(x)$ with the height of the rectangles, and almost half of the students were able to draw connections between $d x$ and the reducing widths of these same rectangles. With this crucial relationship, these same three students, who originally all said $d x$ had no meaning, 
were able to firmly declare multiplication between the integrand and differential was occurring. This great progress in product layer development could be directly attributed to the utilization of guided reinvention the graphical representation of the definite integral afforded.

The use of units also played a vital role in developing a Riemann sum concept image. Much of the progress students demonstrated in understanding the product layer was not prevalent until task two, the accumulation of a fire spreading through a house, was discussed. By using a realistic problem, which included $\frac{f t^{2}}{\min }$ and $f t^{2}$ units, this same collection of students was able to deduce that somehow the transformation the definite integral performed related to the multiplication of min units. Once units were brought to the forefront of discussion, the number of students who believed multiplication occurred within the integral increased from three out of seven, to all seven of the students. While not every student was able to attribute this multiplication by min to the differential in the integral, this reference to a product does show great improvement in eliciting a Riemann sum concept image.

It should also be acknowledged that all of the students involved in this study initially relied on Area Under a Curve or a Fundamental Theorem of Calculus concept image to initially rationalize the necessity of a definite integral in these two contexts. As Jones (2013) and Doughty et al. (2014) have acknowledge, these less prosperous concept images should not be discouraged, but praised. Numerous experts agree that a concept image reflective of a concept's formal definition provide the greatest opportunity for student success (Jones, 2013; Jones, 2015; Meredith \& Marrongelle, 2008; Nguyen \& Rebello, 2011; Oehrtman, 2009; Rösken \& Rolka, 2007; Sealey, 2014; Von Korff \& Rebello, 2012). However, as this study has demonstrated, AUC and FTC concept images, rather than dismissed, can be used as tools to elicit understanding reflective of this more robust Riemann sum concept image. 


\section{LIMITATIONS}

An apparent limitation of these studies is the population group being interviewed. These students all came from the small sample size of one Applied Calculus course at the same university over a two-semester period. Each semester, there were five to six different sections of the course in which the students were enrolled. Each section had between 80 and 100 students enrolled. As it has been said before, it was incredibly interesting seeing the prosperous development these students demonstrated in understanding the variety of layers of the RIF. This was done in spite of seeing no formal presentation of limits in class. Naturally elicited responses may have varied drastically if these students had seen formal instruction on limits, involving its notation and how they may be computed. While these students did demonstrate some levels of difficulty progressing through the exact sublayer of the limit layer, many of these same students were able to successfully navigate through the idea of improved approximations in the approximation sublayer. This difficulty of completely incorporating the infinitesimal nature of the limit reflects and supports claims by many other researchers in the definite integral and limit community (Cornu, 1991; Jones, 2013; Oehrtman, 2009; Sealey, 2014).

It is also important to note that this group of students was self-selected for the study. Every student who volunteered for this study was given the opportunity to be interviewed and no students interested in being included were turned away. I did not collect information on student gender, ethnicity, race, GPA, or class standing from the students in the class or from the students being interviewed. I also did not capture information regarding the students' mathematical background prior to the interviews. This was done to alleviate bias, but as a result, I do not know information regarding attendance or previous mathematic coursework. Some of these students may have missed class the day that relevant material was covered, while others may have received formal 
instruction on limits in a previous math course at the university or at the high school level. Because of this, I am not able to confirm this group of students interviewed accurately reflects the thoughts and ideas of the class as a whole. Of the over 1200 students enrolled over the two semesters, only twelve students were interviewed.

The interviews for the main study were conducted outside of a classroom setting, one on one with the interviewer and interviewee. This format, while done purposefully to gain a better understanding of how individual students were able to view a definite integral through a Riemann sum lens, presents cautions for in-class implementation. Responses received in this setting may not necessarily be reflected of how students interpret a definite integral in a lecture-based classroom setting. Most of the interview discussion was guided by an individual student's thoughts and ideas. In a classroom environment, it would be more likely that discussion would be led by the instructor or by students in a small group setting. With this higher student to instructor ratio, the teacher would play a lesser role in the individual process of guided reinvention that was key to this research. While almost half of the students in this study effectively demonstrated understanding of the limit, product, and summation layers of the RIF, it cannot be assumed that this rate of proficient success would be achieved in a larger classroom setting.

The final limitation that will be discussed is the usage of relatable and highly associable real-life applications of the definite integral. As many experts have already stated, students are much more successful in understanding the necessity of the definite integral when application contexts are more familiar to students (Jones, 2013; Meredith \& Marrongelle, 2008; Orton, 1983; Sealey, 2014; Thompson, 1994). This is a core idea behind RME. In this research, students were presented with situations involving area under a curve and accumulation of rates. These examples were carefully selected to extract previous knowledge that could be used as a tool to guide 
discovery. The area under a curve problem was selected because of the strong graphical and visual aids that could be associated with it. The application problem involving rates and accumulations incorporated realistic units that allowed the prompt and question being asked to relate back to realworld conditions with which students would be familiar. The students in this study frequently saw questions in class involving these two applications and thus were likely to be comfortable with these two scenarios prior to the interviews. Results may have drastically varied if the questions asked involved forces, kinetic energy, or center of mass, or other situations these students would likely be less comfortable quickly associating with integrals. If these students were presented with less familiar or less approachable scenarios, they potentially would have been unlikely to naturally identify the necessity of definite integrals. In this study, many of the students identified the requirement of a definite integral with relative ease. Once this necessity was established, students were then afforded the opportunity to explore the context of the problem and the components of the definite integral to make connections harkening back to the RIF. This likely would not have been possible if a relatively immediate cue for integration was not triggered. 


\section{FUTURE RESEARCH}

My research can easily be expanded by using a larger sample size of students and a sample that better reflects students with formal instruction of limits in a classroom setting. My research has demonstrated that with this group of students, great progress in understanding the RIF occurred through the use of one graphical and one real-life example involving definite integrals. I have shown that not only do these two tasks help students better understand the notion of limits and improved Riemann approximations to obtain an exact solution, but also how meaning can be applied to symbolic components of the definite integrals in order enhance connections to the layers of Sealey's (2014) RIF.

In my study, interviews of only seven students were primarily used. From this sample, it may be difficult to gain information regarding how effective guided reinvention may be for understanding definite integrals through a Riemann sum concept image lens when looking at a large population of students. Ultimately one desired outcome of future research would be to learn how instruction in class could be modified for improved student understanding of definite integrals. To test this instruction reform, additional research on this area should be conducted in a classroom environment and not only in an interview setting. It would also be very interesting to see how these results would be affected if done with students who received formal limit instruction in class. Additional research would allow me to see the impact this computational instruction has on student understanding the limit layer.

This research in a larger classroom setting could be used to determine if any external influences may have an affect on student understanding of the definite integral. In future work, I can dissociate between students that have had formal instruction on the limit in a previous class to see what role this difference in mathematical background may have on the progress through guided 
reinvention. It would also be interesting to note if student attendance in class would influence their reliance on personal critical thinking, as opposed to utilization of previous examples from class.

While on the topic of limits, additional research should be done in a similar vein, but in the context of derivatives. My research was solely focused on student understanding of the definite integral in the context of Riemann sums. This is not the first encounter students have with the notion of limits in calculus. For students in this Applied Calculus course, derivatives were presented in a similar manner to integrals. A real-life situation that required derivatives was presented to the students, allowing them to understand the necessity of the concept prior to a formal introduction. This scenario helped students to see that they needed this new mathematical idea, but they had no idea how to calculate it. Students first explored ways to approximate this idea (since at this point in the lesson, they did not have to tools to calculate a derivative exactly), then how to improve this approximation. Just as with definite integrals, a mathematical leap was then taken to avoid a formal introduction to limits. These students were later presented with the rules necessary to calculate a derivative. So just as with definite integrals, students in this class were presented with the idea and importance of the limit, without any formal notation being presented. While the students in this study did not appear to be hindered by this omission, it is important to see if a similar truth holds in the context of derivatives as well.

Further research could also be conducted on how students utilize units of a problem to justify the use of a definite integral when other symbolic forms of the definite integral are involved. Here, we have only discussed what Jones (2013) refers to as adding up pieces and function matching symbolic forms the definite integral can take in a student's concept image. When these two forms are present, I have been able to demonstrate the impactful role units of a scenario can play in eliciting connections between the components of the definite integral and the product, 
summation, and limit layers of RIF. Jones (2013) has similar research investigating the variety of symbolic forms a student could possess of the definite integral. What is still missing is the way units could interact with other forms such as area between two curves, indefinite integrals, and an integral stretched or flipped by a leading constant multiplier. I have taken two forms in the context of area and accumulations of rates and had students appropriately assign units to further develop understanding of the transformation occurring with definite integrals. Future research could be done to see how the use of units in a realistic situation could affect the way students interpret other forms of a definite integral.

Finally, it could be very interesting to explore how students are able to explain that two different integrals both result in an area. One unintentional outcome of this research was presenting students with two ways area could be encountered and how the definite integral plays a role in their development. In the first example, a unitless length and width were used to calculate area of rectangles that would then be summed to approximate the area of a given region. In the second example, a rate of fire spreading was given, and students were tasked with calculating the total area of house engulfed. In both scenarios, students were given information, tasked with calculating an area, and ultimately accomplished this goal through the use of a definite integral. Even though the given information in both situations was completely different, the result of a definite integral was the same quantity. During the interviews, it was never brought to the students' attention how an integral could be applied to two completely different sets of given information and both result in an area calculation. I hope to be able to conduct future research investigating student response on this potential cognitive conflict. In this environment, a student would have at their disposal the visual tools of the area under a curve problem, the real-life context of an accumulation of rates, and the units of the definite integral and its components. 


\section{TEACHING IMPLICATIONS}

Here, I will be discussing teaching implications in two very different contexts. In the first, a philosophical discussion will be had on how this research should influence the way instruction on definite integrals in a calculus course should be conducted. Following this, I will put forward potential lessons that could be incorporated into curriculum instruction to help achieve the previously discussed holistic goals. A sample activity reflecting the ideas put forth by the potential lesson will then be presented. While the comments and suggestions in the section are based on the results represented in my research, ultimately, they are my own personal opinions on how teachers can be most impactful in facilitating the learning of definite integrals.

\section{A Philosophical Outlook on Teaching Definite Integrals}

I would like to begin by affirming the claims made by Jones (2013) that students wear many hats when thinking about definite integrals. The concept image a student demonstrates while discussing a definite integral may represent a very poor reflection of the knowledge he or she truly possesses. Every student I have discussed here initially relied on a more remedial concept image founded in rote memorization. Every student interviewed also was able to present, in some capacity, a more robust understanding, better reflective of a Riemann sum concept image at some point in the interview. Before a teacher attempts to take on the role of eliciting this deeper understanding, they must first be cautious not to assume naivety on the student's behalf. When measuring student mastery of definite integrals, it is unproductive to accept a student's response that is demonstrative of an AUC or FTC concept image as the extent of understanding. Instead, this should be a cue for the instructor to discover ways to elicit layers of the RIF from said student.

As I have done, instruction can utilize situations where necessity of a definite integral is memorized and use it as an opportunity to bolster learning. In the particular Applied Calculus class 
from where the students interviewed here were pulled, large emphasis was placed on knowing when a definite integral was an appropriate tool to answer a given prompt and when it was not. So, for many of the students interviewed, they knew a definite integral was required to answer the given situations presented with relative ease. Once it was agreed upon that a definite integral was required, much of the burden of getting a correct answer was lifted. Now, a shift in discussion could be made, placing all emphasis on why this integral would result in the correct answer. If I had reproved student responses that were based on AUC or FTC concept images, this shift to discussing why would not have been able to occur. Since very seldomly a Riemann sum concept image was naturally evoked by the students in this research, providing an explanation for an answer they already knew proved to be a great tool in guiding students towards this stronger definite integral representation.

This student population received no formal limit instruction in class. In these interviews, the students were also never asked to perform any computations explicitly involving a limit. The claims made in this work are not designed to combat those of others, such as Oehrtman (2008), on the importance of instruction relating to the limit. I do not suggest that formal instruction of limits serves no purpose in enhancing student understanding of integrals. I simply propose the hypothesis that it may not necessarily be a requirement for a student to produce a proficient demonstration of Sealey's (2014) limit layer. The crucial instruction must focus on the purpose and idea of the limit in order for it to be properly encapsulated by the student. It may be that computing limits and incorporating its definition into a curriculum provides less benefit to students than simply emphasizing the role its idea plays in repeatedly improving approximations.

Just as recent research suggests, struggles with the idea of limit do not often appear until infinity and infinitesimals are introduced (Cornu, 1991; Oehrtman, 2008; Oehrtman, 2009). This 
dichotomy in ease of improving approximations and difficulty in making an approximation exact should warrant a distinction on the instructor's behalf as well. If a student quickly demonstrates a strong understanding of how to make an approximation better, it should not be assumed that this student fully understands the extension of obtaining an exact solution. Conversely, if a student has trouble encapsulating the infinitesimal size $\Delta x$ approaches in a Riemann sum, it should not be assumed that a student would also have difficulty with the beginning stages of improving a Riemann sum through the use of more and smaller intervals. It is crucial for instruction to split the limit layer into the approximation and exact sublayer. This split will allow instruction to properly place focus when common difficulties arise. As stated, ultimately the vast majority of issues arise when students are forced to leave the real number line. Chase and Jeremy specifically demonstrated great difficulty maneuvering from a mindset with very small intervals to infinitesimally small intervals. Others have suggested that the trouble students encounter with limits often is rooted in student difficulty encapsulating infinity and infinitesimals (Cornu, 1991; Oehrtman, 2008; Oehrtman, 2009). With this knowledge, supported by my research, instruction should be sensitive to understanding the cause of the potential cognitive conflict that exists within the exact sublayer.

The use of previous knowledge a student possesses outside of the classroom can also be used as a valuable tool to better understanding definite integrals. The guiding (pun intended) feature of Realistic Mathematics Education is the use of real-life examples that students have had previous experience with (Gravemeijer \& Doorman, 1999). Utilizing previous knowledge helps to bridge the gap the abstract nature of calculus may present. Discussion of position, velocity, and acceleration are often some of the first ways students are introduced to applications of definite integrals. I believe the role these ideas play in student success in understanding the definite integral 
result from how natural it is to think about and discuss a ball being thrown in the air or driving a car on the highway. With these examples, students already have an intuitional understanding of the mechanics involved. For instance, if a student knows they are driving at $60 \mathrm{mph}$ for two hours, it will likely come very naturally to him or her how to calculate the total positional change over that two-hour period. This could then be used as a building block to introduce students to a situation where the velocity of the car varies and a total distance traveled is still the ultimate goal (Sealey \& Engelke, 2012).

With realistic situations often times also comes relatable and approachable units. For this particular study, many of the students knew the units of the given function were $\frac{f t^{2}}{\min }$, the units of the required solution were $f t^{2}$, and that this transformation occurred through the use of an integral. Often times, all of this background knowledge was demonstrated by the student prior to giving any explanation for why a definite integral was the proper mathematical operation required to obtain the correct answer. Now that the students had these tools, they began to rationalize how the integral symbolized this transformation. In doing so, students hypothesized by attributing units to the differential, performing the operation of multiplication within the definite integral, and even properly connecting this product to the integrand and differential. It is important for an instructor to utilize as many tools in the knowledge toolbox a student already possesses in order to heighten the likelihood of student understanding reflecting a Riemann sum concept image.

Another tool instruction can utilize to overcome the abstractness of definite integrals is of a visual nature. I have already gone to great lengths discussing the drawbacks of an overreliance on an area under a curve representation of the definite integral. It should still be said that this overrepresentation can prove to be a valuable instrument in improving student understanding of the definite integral from a Riemann sum perspective when done correctly. As with the real-life 
application example, many of the students interviewed had already presented an understanding that a definite integral was necessary to calculate the area under a given curve. The tools students had already developed to help approximate this calculation were then used the facilitate connections between Riemann sums and the definite integral. Instead of rebuking claims students made that were reflective of an AUC concept image, I used them as a way to guide discussion on why an area under a curve resulted from the definite integral. While the extent of this discussion varied from student to student, it again showed that instruction that utilized visual aids to help guide a student's explanation/discovery was prosperous.

Ultimately, it can be summarized that students frequently exhibit difficulty in demonstrating a Riemann sum concept image of the definite integral. When this understanding is not present, or other common concept images are used in its stead, an instructor can view this shortcoming in two manners. These claims made by the student can be discarded in hopes that a more prolific level of understanding can be elicited through further instruction, or as this research suggests, these statements can be used as building blocks to help guide a student to a deeper understanding. Whether an active or passive decision, students in these interviews had chosen to rely on images based in rote memorization. Rather than dismiss this choice, teachers are now afforded the opportunity to shift facilitation away from knowing that a definite integral is required to understanding why a definite integral is required. By applying this refocus in instruction, not only does this heavier emphasis on why provide ample opportunity to further develop a Riemann sum concept image, but the students now have more tools to do so than they did when originally discovering the idea of definite integrals. 


\section{An Example of how to Elicit a Riemann Sum Concept Image}

The curriculum I suggest here will be best suited for a scenario where students have already progressed through a large portion of the definite integral unit in their calculus sequence. The following lesson should be used to address the situation where students have begun to utilize memorization as the key tool for understanding when an application problem requires a definite integral. Following this generic description of a lesson, I will be providing an example activity that instructors could use to implement this lesson. Now that these students have a strong foundation in understanding when a definite integral should be used, this lesson can be used to revisit a definition-based rational for the necessity of a definite integral. I will describe the process I hope for students to follow, essentially guided reinvention of an idea they had learned before, but now with the tools and assuredness needed to enhance understanding. At multiple points in the lesson, there will be the opportunity for connections to be made between the definite integral, its Riemann sum definition, and various representations of this calculus concept. Because of the varying levels

of understanding students will enter in the lecture, there may be multiple opportunities for the same "aha moment" throughout the lesson.

Students are presented with an arbitrary graph of a curve and will be asked to provide a method for calculating the area under the given curve from point $a$ to $b$. It can be presumed that at this stage in the unit, students feel comfortable knowing a definite integral is the required tool to accomplish this goal. Now that the students have demonstrated how to obtain the correct answer, the rest of the unit can redirect focus to understanding why this definite integral results in the area under the curve. When definite integrals were originally discussed in class, the students may not have been afforded the luxury of already knowing the answer to the problem. Their attention 
originally would have been split between understanding the process and understanding what the ultimate solution should be. Now, all focus can be redirected to why instead of how.

Once it has been established that the integral results in the desired area, students will then be instructed that this graph represents a rate. The students will be asked, knowing this context, what does the definite integral represent? Again, it should be assumed that since these students have already been introduced to and are comfortable with a variety of definite integral applications, there should be minimal difficulty expressing understanding that in this new context, the integral would also result a total accumulation from $a$ to $b$. Up to this point in the lecture, there likely will be no information that has not been readily accessible through the use of an AUC or FTC concept image. There have been connections made from area under a curve and accumulations of rates to definite integrals, common place in many calculus courses.

Discovery has the potential to occur when units are introduced. Students will first be asked to establish what the units of the given function and resulting definite integral are. If it has not yet occurred, this question should incite students to believe that there is some sort of transformation occurring when a definite integral is applied. Ideally, students will be able to establish that the units for the function and units of the resulting integral are different. Students will now be tasked to justify with algebra this change in units. This question will likely trigger the notion that somewhere, multiplication is occurring. For those students relying on rote memorization, this would be the first indication in this lesson that a product of some sort could be involved in the definite integral. My study suggests that some students demonstrate difficulty establishing where this multiplication should occur. This provides a wonderful opportunity for students to hypothesize where, if anywhere, multiplication is occurring within the definite integral's symbolic components. Potential responses could be between the integral symbol and the integrand, between the integrand 
and the differential, or not at all. As a result of this common struggle, there will now be a shift away from the application scenario and back to the graphical context. This will be done in hopes to provide students more opportunities to build a case in support of their hypothesis, or to prove their hypothesis incorrect.

In order to develop tools to assist in this leap to incorporating multiplication into a definite integral, the following question could be asked to better understand how units play a role in the definite integral. 'On our graph's axes, where would we label the units of our rate and where would we label the units we must multiply by in order to obtain the units of our integral?" Hopefully it is clear to the students that the units of our function fall on the $y$-axis. It also should not be too large of a leap that the $x$-axis represents the units we need to multiply by. Thus, a small group of students should be able to work together to readily assign these units to the $x$-axis.

Once units have been assigned to the $x$ and $y$ axes, it will be reiterated that the definite integral represents total accumulation and that multiplication must occur in order to obtain the resulting units. If not naturally prompted, students can be given the hint that when length $(x)$ and height $(y)$ are multiplied, the result gives us the area of a rectangle. This step in the lesson allows for two potential scenarios. A student can either be introduced to the relationship between integrals and Riemann rectangles for the first time, or this can trigger a preexisting connection between the two that will help guide development of a Riemann sum concept image that may already exist deep inside the student's mind.

It should be stated to the students that the width of the single rectangle make sense to start at $a$ and end at $b$. Difficulty may arise in determining where information about the height of the rectangle should be drawn. This allows for an opportunity for the students to hypothesize how high the rectangle should be to be equivalent to the area of the region under the curve. With instruction, 
it should be made apparent that ultimately this placement of the height of the rectangle is subjective, with many possible excellent guesses. In particular, a guess for this height may be the height of the function at $a$ or at $b$. For simplicity of the remaining lesson, we will use the height at $a$, or the "Left-hand Side" of our region. A short explanation can be inserted here about why the language, "Left-hand Side" makes sense.

Now students are provided with a visual representation of units being multiplied, a graphical tool to gauge why this is simply an estimate, and a visual aid to justify whether this estimate is too large or too small. Students can now theorize why using only one height, and in turn once rectangle, results in a good estimation of the area and the total accumulation, but not a great one. The hopes are that students draw the conclusion that the rate at $a$ is being used to approximate the rate of the entire interval. The rate in this approximation is assumed to be constant, which it clearly is not. It would be better to periodically use more rates at different intervals so one rate is used to approximate over a smaller interval. Students could then be asked to use four rates that the curve provides instead of just one. This will be done with the reminder that the rate chosen should be the rate at the left bound of each region for consistency. Students are now afforded the opportunity to determine what this new approximation looks like graphically. Previously, one rate was used to establish one rectangle over the entire interval. Now, the region will be broken up into four sections, each with their own left-hand rectangle representing the total change over that interval. The four approximations can then be summed to result in a new, better approximation. By using only one rectangle and then four, students are afforded the opportunity to visually see how more rectangles result in less error, encompassing the approximation sublayer of the Riemann sum limit layer. This is done without the use of an abundance of calculations that could distract from the purpose of reducing interval size. 
Even with just these two approximations, it should be clear to students that more rectangles over smaller intervals result in better approximation. Before connecting to the exact sublayer and how these approximations can be improved to the point where an exact area/exact population is derived, this is a fantastic opportunity to jump back to the symbolic construction of the definite integral to see in if any additional connections can be made to the product layer. In doing so, students may also be able to attribute some summation meaning to the integral symbol. The potential connections at this stage of the activity could vary widely. Some students may be able to hypothesize the $d x$ could represent the widths of the rectangles and that this is being multiplied by $f(x)$. If this connection between the integrand and differential is made, there is a strong possibility that the student will also be able to attribute the units of the $x$-axis to $d x$ as well.

With connections back to the symbolic construction of the definite integral now even more likely, questions like, "how big is $d x$ ", "What size intervals should be used to get an exact answer", "How small can the intervals be", "How many intervals can we have", and "How many intervals must we use to gain an exact answer" can now be asked. It is understandable that there still will be inconsistencies at this stage because of the complexities involved with definite integrals. The hope is that at least some of these exact sublayer questions can provide the opportunity for constructive conversations amongst the small groups, ultimately allowing students to develop their Riemann sum understanding of the definite integral. 


\section{Definite Integral Lesson}

1. How would you calculate the area underneath this curve, $f(x)$, from $x=3$ to $x=7$ ? (Hint: You are not being asked to get a numeric answer, just the setup)

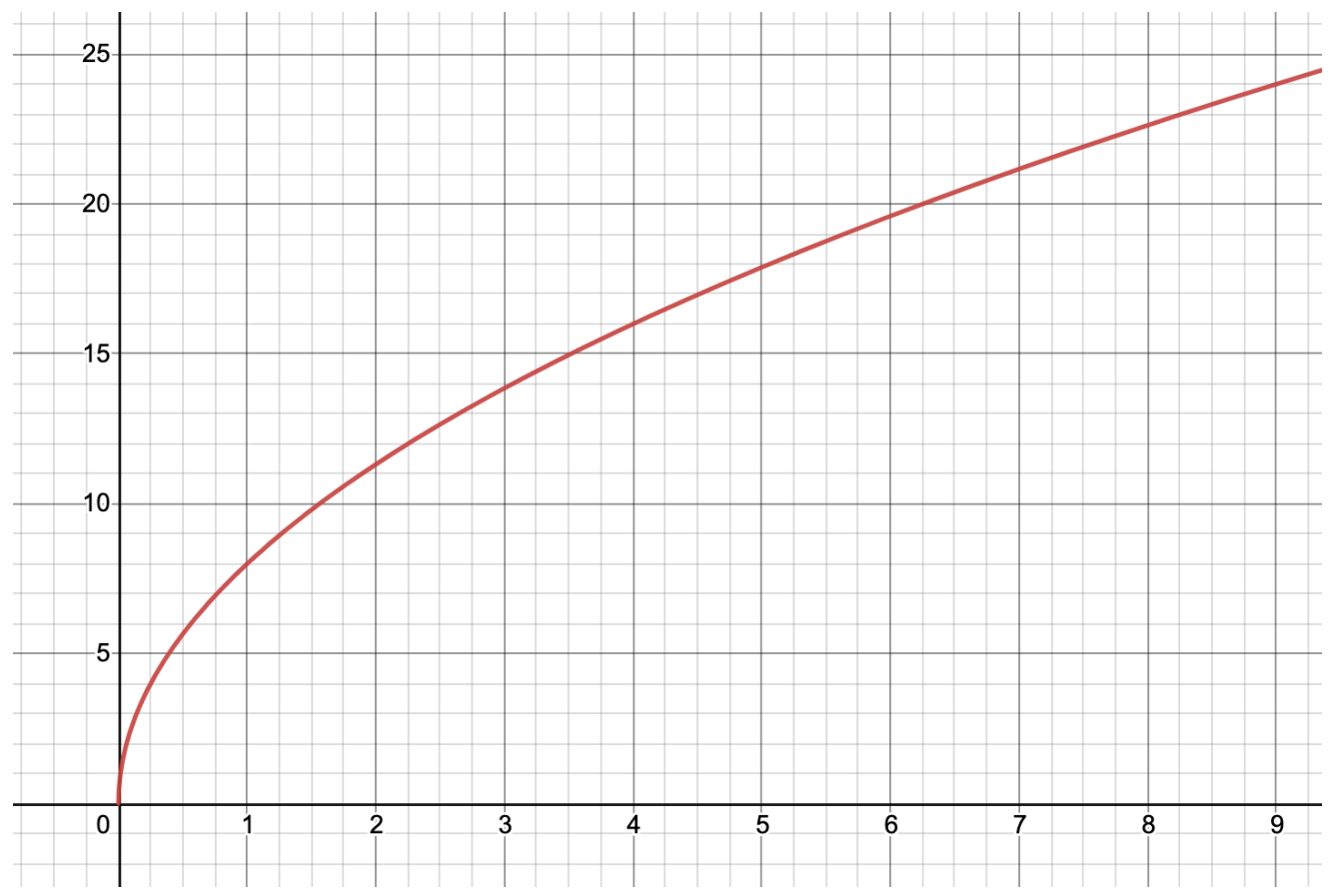


2. For the following questions, assume that $f(x)$ represents the rate at which the population of a city is growing each year.

a. What are the units of $f(x)$ (writing this as a fraction will help later)?

b. What would $\int_{3}^{7} f(x) d x$ represent if we know that $f(x)$ is the population growth rate?

c. What are the units of $\int_{3}^{7} f(x) d x$ ?

d. Notice the units of our integral are different than the units of $f(x)$. What should the units of our $f(x)$ be multiplied by to give us the units our integral gives us? 
3. So, it makes sense that the units $\frac{p p l}{\text { year }}$ could be multiplied by years to give us the resulting ppl units we know our integral gives us.

a. What, if anything, represents the units $\frac{p p l}{y e a r}$ in our integral, $\int_{3}^{7} f(x) d x$ ?

b. What, if anything, represents the units of year in our integral. $\int_{3}^{7} f(x) d x$ ?

c. Do you think any multiplication is occurring within the integral that could represent this idea of multiplying $\frac{p p l}{y e a r}$ by year (you may or may not)? 
4. Things may be getting pretty confusing already, so let's shift back to the graph we originally started with. Now that we have talked some about units, label the units for the $x$-axis and $y$-axis if our function is the rate of population growth each year.

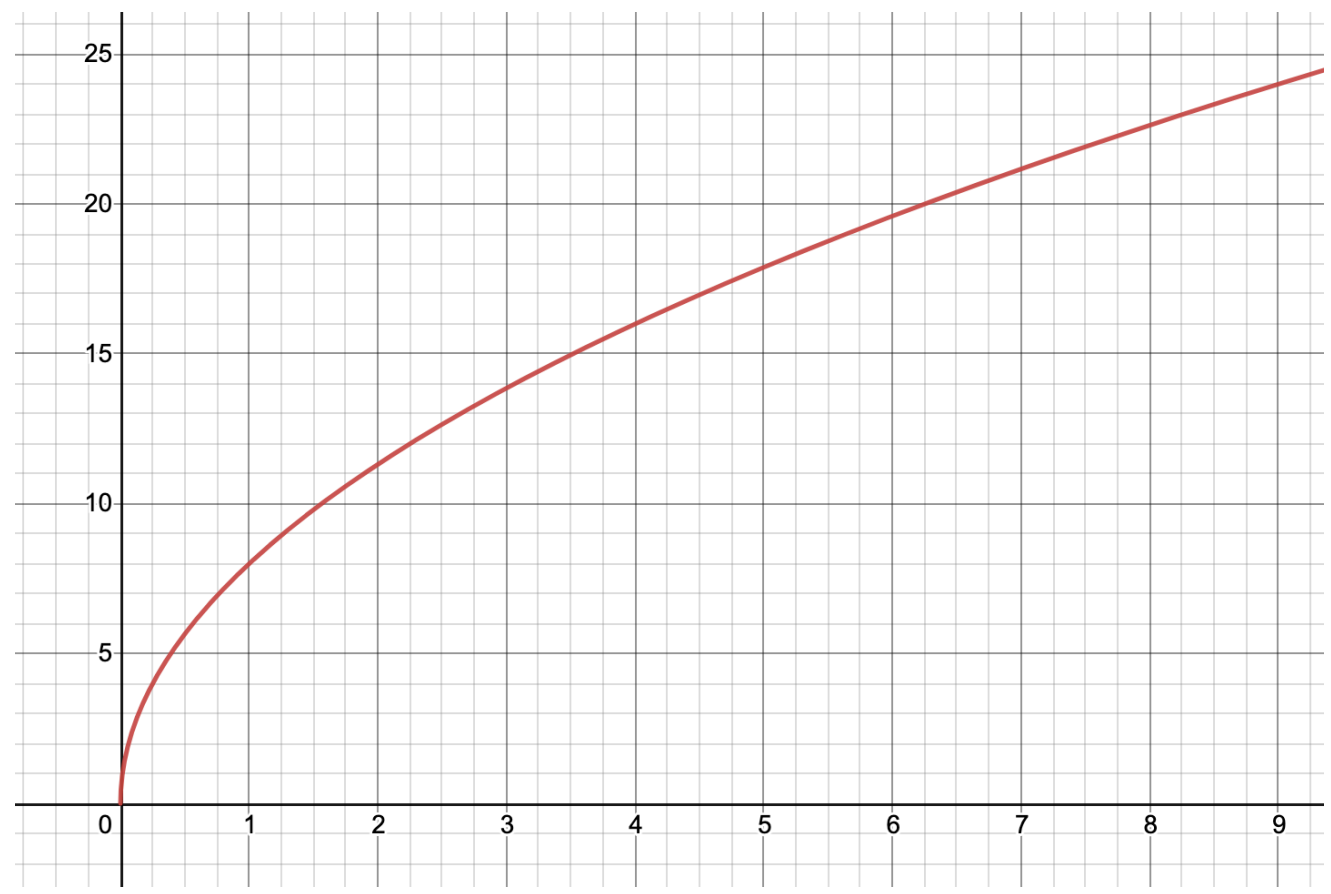


It's not a coincidence that the units of our two axes are the same as the units we had previously discussed! Before, we had thought $\frac{p p l}{y e a r}$ and year could be multiplied to give us the units that $\int_{3}^{7} f(x) d x$ has. Let's see what this multiplication could look like graphically. Think back to your days in a geometry class. What do we get when we multiply length times width?

In this scenario, hopefully it makes sense that the interval on the $x$-axis could represent a width, and the interval on the $y$-axis could represent the height of one big rectangle. Now it's time to make some big connections! We have hypothesized that multiplication of $\frac{p p l}{\text { year }}$ and year had to occur somewhere in our integral to give us the units $p p l$. $f(x)$ has the units $\frac{p p l}{y e a r}$ and there still may be confusion about what, if anything, has the year units in our integral. But when we look at these units graphically, it is very clear to see that this multiplication of $\frac{p p l}{y e a r}$ and year is EXACTLY what is happening when we multiply our $x$-axis values by our $y$-axis values to give us the area of a rectangle. And in fact, this rectangle, as we will see, has very strong connections to the multiplication that is occurring in the definite integral!

5. Now, try to draw this big rectangle that we hope will help guide us to better understanding the integral. Remember, we think this rectangle has something to do with the integral, which means this rectangle should resemble the area under the curve from 3 to 7. (Hint: You will need to guess what the height of your rectangle should be)

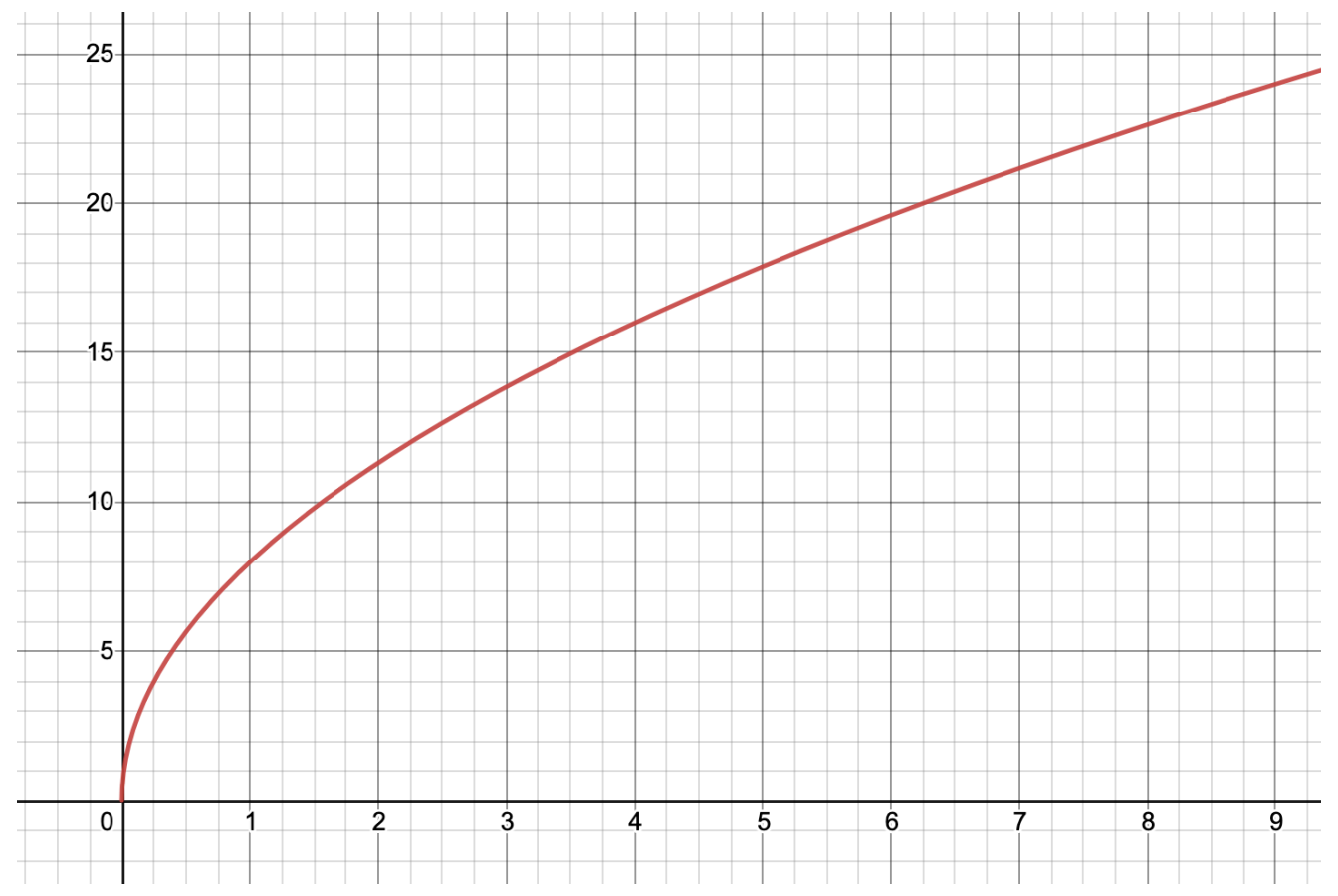


As you can see, there are a lot of possible guesses for what this height should be. The reason for this is we are only approximating the area under the curve with this one big rectangle, and there are many ways we can do this approximation. We could eyeball the graph to try to match the area of the two shapes the best we can, we could use the function value at 5 because it's halfway between 3 and 7, we could use our function value at our starting time of 3 , or we could use the function value at our ending time 7 . These, and many more options are fantastic guesses for what the height of our rectangle could be.

6. For consistency from here on, let's use the function value when $x$ is 3 to draw out rectangle. Try to draw one big rectangle from 3 to 7 with a height at $f(3)$.

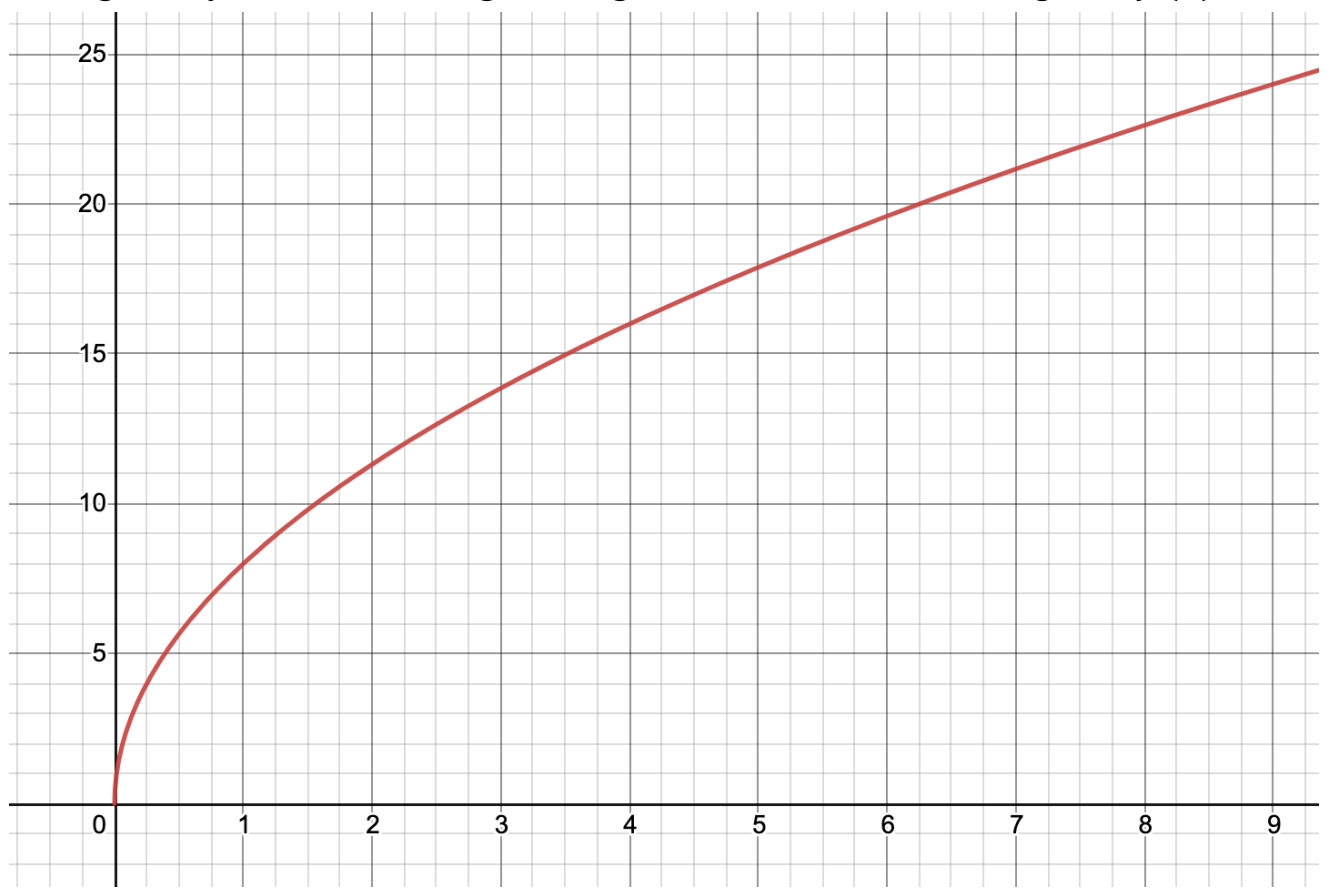

This rectangle is called a left-hand approximation. The reason it is called left-hand is because the left corner of the rectangle touches the graph. If we were to have used the function value at 7 for our height, this would have given us a right-hand approximation because the right side of our rectangle is touching our graph.

7. It should be clear that the area of this rectangle is definitely only an approximation and is not exactly the same as the area under our curve. Is this approximation an under approximation or an over approximation? How do you know? 
8. For our rectangle, we are assuming the rate at which our population is growing stays the same the entire time from 3 to 7 . This is why the top of this rectangle is a flat line. We know this isn't actually true though because from 3 to 7 , our function value is constantly changing (notice the function is always increasing). Instead of assuming our rate is the same for the entire interval, what is a way we can make our approximation better? While coming up with ideas, think about how we can use rectangles (hint: notice the plural) to better approximate our area or how we can better represent the rate of population growth changing as time progresses. 
9. Instead of assuming the rate from 3 to 7 is the same the entire time, let's assume the rate is constant for just the first year. Then we'll reevaluate at this time and assume the rate for the entire next year is the same as the given rate at time $=4$. This idea will continue until we have reached the end of our time interval. Try to draw what this new approximation would look like with rectangles. The first rectangle is drawn for you (hint: there should be 4 rectangles when you are done. The top left corner of each rectangle should be touching the graph)

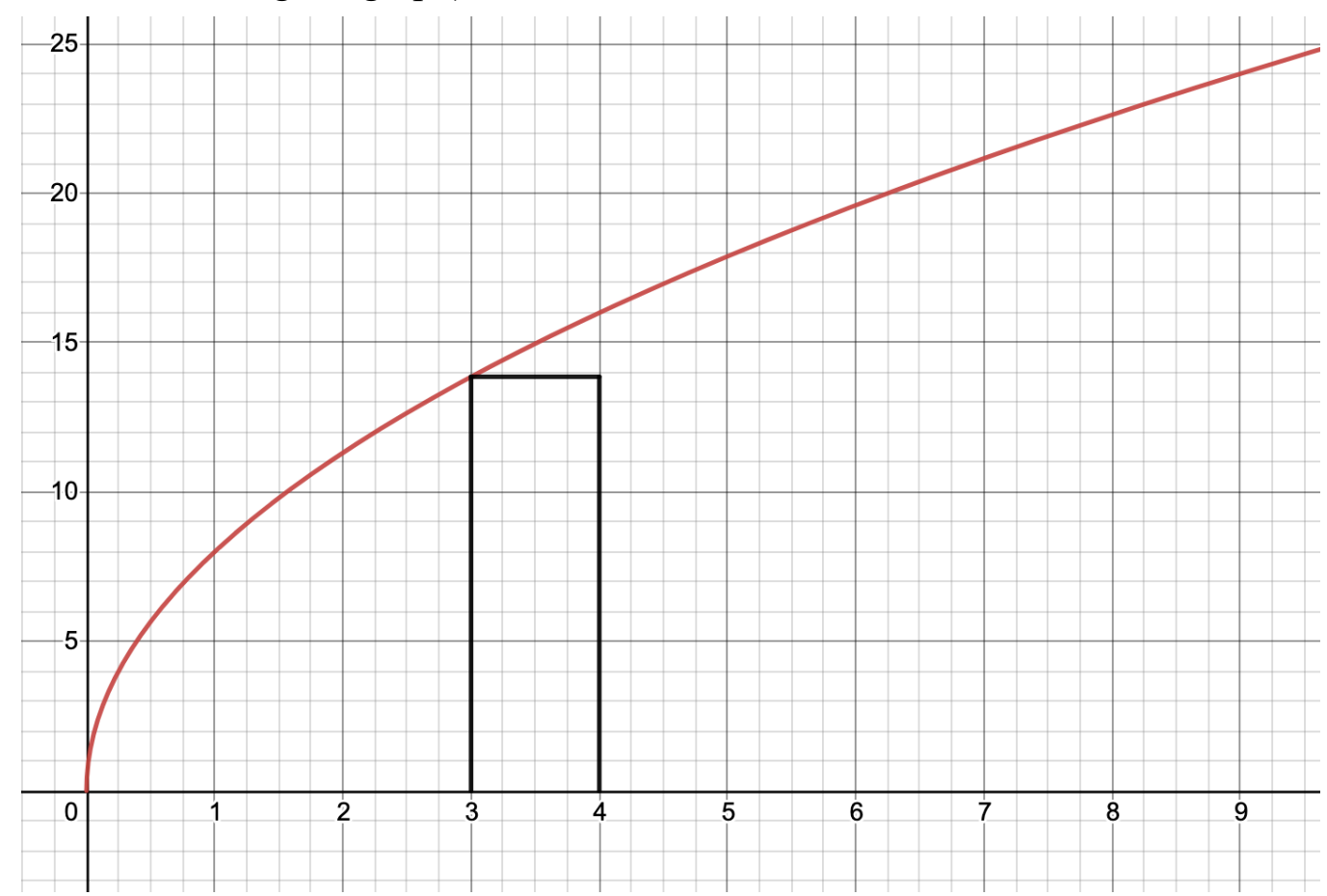

a. Explain why this gives a better approximation of the area under the curve than when only one rectangle was used.

b. Explain why this gives a better approximation of the total population than when we assumed the population changed by the same rate (the rate at time $=3$ ) the entire time.

c. How do you think you could get an even better approximation? 
10. Let's try to draw connections back to the definite integral now, $\int_{3}^{7} f(x) d x$.

a. Now that we have this picture with 4 rectangles, what in the rectangles could the $f(x)$ represent?

b. Is there anything in our rectangles that the $d x$ could represent?

11. In our example, we have talked about the units $\frac{p p l}{y e a r}$ and year multiplying. We have also talked about the length and widths of our rectangles multiplying to give us our areas as well.

a. Is there anything that you think may be multiplying in your integral?

b. Does this answer match what you have stated about what $f(x)$ and $d x$ represent for question 10 ? 
12. Time to bring everything together. We have talked about how more rectangles give us better approximations.

a. How many rectangles would we need to get the exact area under the curve (this is ultimately the same thing as the definite integral)?

b. What would the widths of these rectangles be to get an exact area under the curve?

c. At the beginning of today, we said that $\int_{3}^{7} f(x) d x$ gives us the EXACT area under our curve. We have also talked about how $f(x)$ could represent the height of each our rectangles used. If more and more rectangles give us better approximations, then that means we would need an infinite number of them to get the best possible answer (this is our exact area!). What would the widths of these rectangles be to squeeze an infinite number of them between 3 and 7 ?

13. After this discussion, what can you tell us about $d x$ ? While answering these questions, keep in mind the number of rectangles you would need to get an exact answer.

a. Do you think $d x$ has any units in our example? If so, what are they?

b. Do you think $d x$ has a "size" to it? If so, how big (or small) is $d x$ ?

c. Do you think $f(x)$ and $d x$ multiply each other in $\int_{3}^{7} f(x) d x$ ? 


\section{REFERENCES}

Braun, V., \& Clarke, V. (2006). Using thematic analysis in psychology. Qualitative research in psychology, 3(2), 77-101.

Bressoud, D. M., Mesa, V., \& Rasmussen, C. L. (Eds.). (2015). Insights and recommendations from the MAA national study of college calculus. MAA Press.

Cornu, B. (1991). Limits. Advanced mathematical thinking (pp. 153-166) Springer.

Davis, R., \& Vinner, S. (1986). The notion of limit: Some seemingly unavoidable misconception stages. Journal of Mathematical Behavior, 5, 181-203

Doughty, L., McLoughlin, E., \& van Kampen, P. (2014). What integration cues, and what cues integration in intermediate electromagnetism. American Journal of Physics, 82(11), 10931103.

Gravemeijer, K. (1999). How emergent models may foster the constitution of formal mathematics. Mathematical Thinking and Learning, 1(2), 155-177.

Gravemeijer, K., \& Doorman, M. (1999). Context problems in realistic mathematics education: A calculus course as an example. Educational studies in mathematics, 39(1-3), 111-129.

Hall, W. L. (2010). Student misconceptions of the language of calculus: Definite and indefinite integrals. Proc. 13th special interest group of the Mathematical Association of America on research in undergraduate mathematics education.

Hough, S., \& Gough, S. (2007). Realistic Mathematics Education. Mathematics Teaching Incorporating Micromath, 203, 34-38.

Jones, S. R. (2013). Understanding the integral: Students' symbolic forms. The Journal of Mathematical Behavior, 32(2), 122-141.

Jones, S. R. (2015). Areas, anti-derivatives, and adding up pieces: Definite integrals in pure mathematics and applied science contexts. The Journal of Mathematical Behavior, 38, 928.

McCarty, T. \& Sealey, V. (2019). What is a Differential? Ask Seven Mathematicians, Get Seven Different Answers. Proceedings of the 22nd Annual Conference on Research in Undergraduate Mathematics Education. Conference on Research in Undergraduate Mathematics Education, Oklahoma City, OK.

Meel, D. E. (2003). Models and theories of mathematical understanding: Comparing Pirie and Kieren's model of the growth of mathematical understanding and APOS theory. CBMS Issues in Mathematics Education, 12, 132-181. 
Meredith, D. C., \& Marrongelle, K. A. (2008). How students use mathematical resources in an electrostatics context. American Journal of Physics, 76(6), 570-578.

Nguyen, D. H., \& Rebello, N. S. (2011). Students' understanding and application of the area under the curve concept in physics problems. Physical Review Special Topics-Physics Education Research, 7(1), 010112.

Oehrtman, M. (2008). Layers of abstraction: Theory and design for the instruction of limit concepts. Making the Connection: Research and Practice in Undergraduate Mathematics, MAA Notes Volume, 73, 65-80.

Oehrtman, M. (2009). Collapsing dimensions, physical limitation, and other student metaphors for limit concepts. Journal for Research in Mathematics Education, 396-426.

Orton, A. (1983). Students' understanding of integration. Educational Studies in Mathematics, 14(1), 1-18.

Prawat, R. S., \& Floden, R. E. (1994). Philosophical perspectives on constructivist views of learning. Educational Psychologist, 29(1), 37-48.

Rösken, B., \& Rolka, K. (2007). Integrating intuition: The role of concept image and concept definition for students' learning of integral calculus. The Montana Mathematics Enthusiast, 3, 181-204.

Sealey, V. (2006). Definite integrals, Riemann sums, and area under a curve: What is necessary and sufficient. In Proceedings of the 28th annual meeting of the North American Chapter of the International Group for the Psychology of Mathematics Education (Vol. 2, No. 1991, pp. 46-53).

Sealey, V. (2014). A framework for characterizing student understanding of riemann sums and definite integrals. The Journal of Mathematical Behavior, 33, 230-245.

Sealey, V., \& Engelke, N. (2012). The Great Gorilla Jump: An Introduction to Riemann Sums and Definite Integrals. MathAMATYC Educator, 3(3), 18-22.

Sealey, V., \& Thompson, J. (2016). Student interpretation and justification of" backward" definite integrals. In 19th Annual Conference on Research in undergraduate Mathematics Education, Pittsburgh, PA.

Sfard, A. (1991). On the dual nature of mathematical conceptions: Reflections on processes and objects as different sides of the same coin. Educational studies in mathematics, 22(1), 136.

Sfard, A. (1992). Operational origins of mathematical objects and the quandary of reification-the case of function. The concept of function: Aspects of epistemology and pedagogy, 25, 5984. 
Strauss, A., \& Corbin, J. M. (1997). Grounded theory in practice. Sage.

Tall, D. \& Vinner, S. (1981). Concept image and concept definition in mathematics with particular reference to limits and continuity. Educational Studies in Mathematics, 12, $151-169$.

Thompson, P. W. (1994). Images of rate and operational understanding of the fundamental theorem of calculus. Learning mathematics (pp. 125-170) Springer.

Treffers, A. (1993). Wiskobas and Freudenthal: Realistic mathematics education, Educational Studies in Mathematics, 25, 89-108.

Treffers, A., \& Beishuizen, M. (1999). RME in the Netherlands. Issues in teaching numeracy in primary schools.

Von Korff, J., \& Rebello, N. S. (2012). Teaching integration with layers and representations: A case study. Physical Review Special Topics-Physics Education Research, 8(1), 010125. 


\section{APPENDIX A: Pilot Study Interview Protocol}

\section{Question 1:}

- How would you go about finding the area of the following region above the $\mathrm{x}$-axis

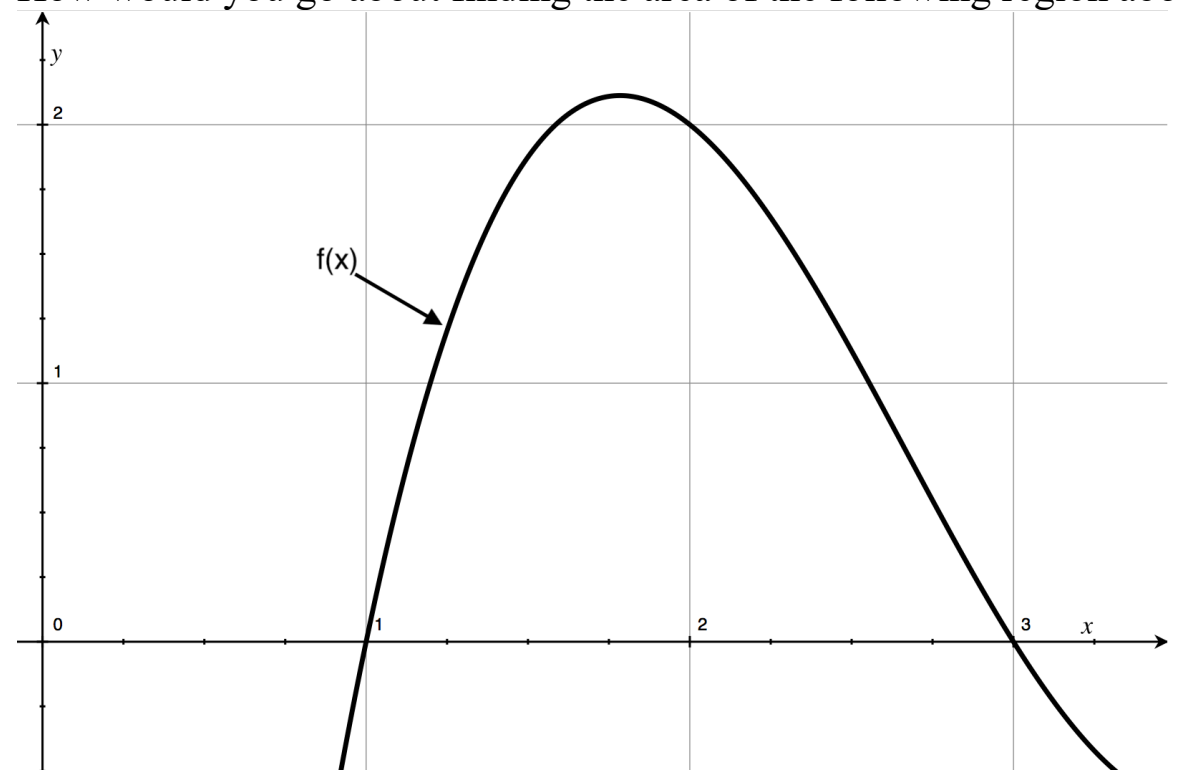

- How do you know?

- If a complete definite integral is prompted then ask what each aspect of the notation represents.

- If an incomplete definite integral is prompted then ask what each aspect of the notation represents and prod at the mistakes.

- If no definite integral is prompted then discuss how it could be approximated with rectangles

- What is an answer that would definitely be wrong if you got it

- What is the area if $f(x)=x^{3}-8 x^{2}+19 x-12$ ? Does this answer make sense, why?

$\circ$ If not prompted point out grid on graph 


\section{Question 2:}

A fire starts in a 5000 square foot house that spreads at a rate of $f(t)=e^{\frac{t}{2}}$ square feet per minute, $t$ minutes after the fire begins

- How would you calculate the amount of the house engulfed by the fire at time $a$ ?

o If a complete definite integral is prompted then ask what each aspect of the notation represents.

- If an incomplete definite integral is prompted then ask what each aspect of the notation represents and prod at the mistakes.

- Can you use the definite integral structure to help determine what units of the definite integral will be?

- How much of the house is engulfed after 14 minutes?

Does this answer make sense, why?

- What is an answer that would not make sense?

- How much of the house is left after 15 minutes?

$\circ \quad$ Does this answer make sense, why?

- What is an answer that would not make sense?

- How much of the house is left after 16 minutes?

- Does this answer make sense, why?

- What is an answer that would not make sense?

- How long will it take for the entire house to be burned?

- Does this answer make sense, why?

$\circ$ What is an answer that would not make sense?

- If the units for the x-axis is "meters" and the units for the y-axis" is "meters", what are the units for the definite integral.

○ Do any aspects of the definite integral notation support this conclusion? 


\section{APPENDIX B: Dissertation Study Interview Protocol}

\section{Question 1:}

- How would you go about finding the area of the following region above the x-axis

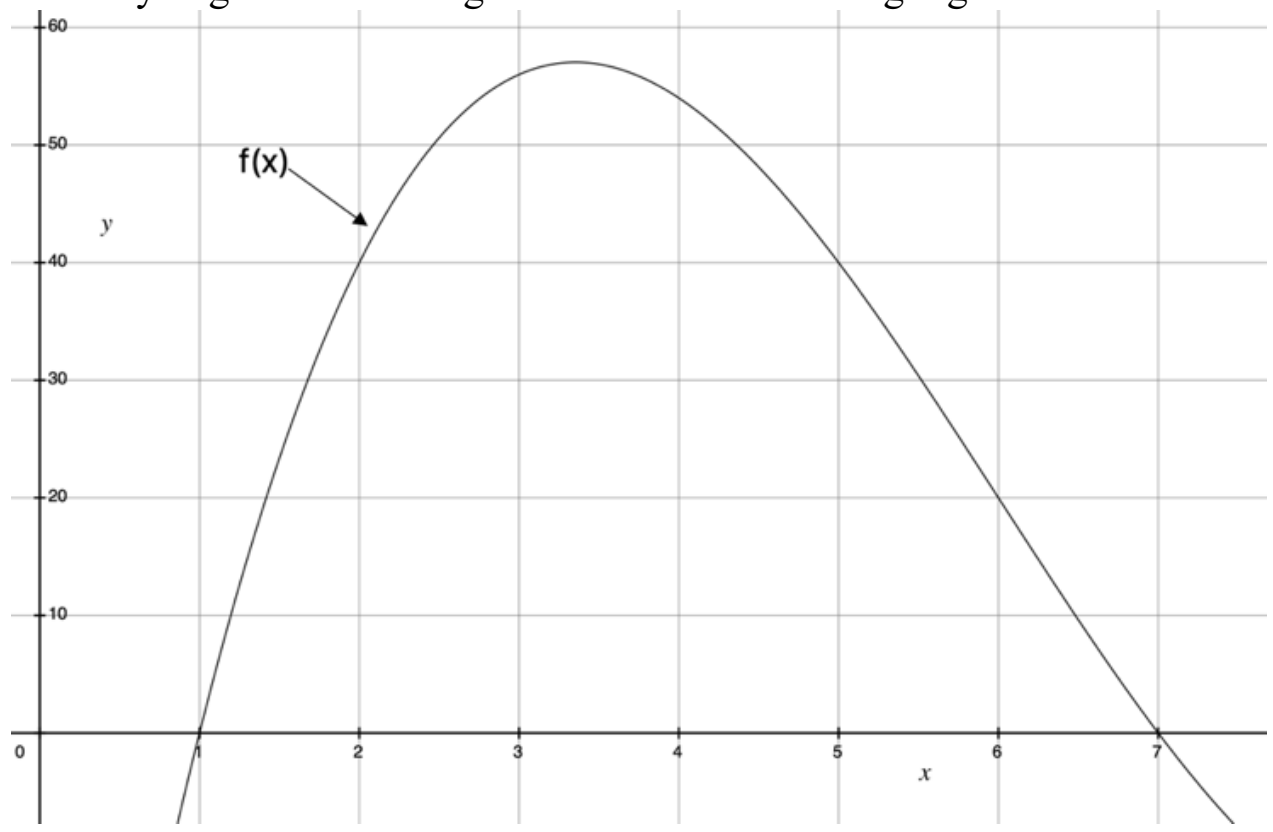

- How do you know?

- If a complete definite integral is prompted then ask what each aspect of the notation represents.

- The integral symbol

- bounds

- $\mathrm{f}(\mathrm{x})$

- $\mathrm{dx}$

- product between $\mathrm{f}(\mathrm{x})$ and $\mathrm{dx}$

O If an incomplete definite integral is prompted then ask what each aspect of the notation represents and prod at the mistakes.

- If no definite integral is prompted then discuss how it could be approximated with rectangles.

- What do you think the area will be?

- Why do you think this will be the area?

- If a connection to the Riemann sum is made, ask what relationship the aspects of the rectangles have to the definite integral notation.

- What is an answer that would definitely be wrong if you got it?

- What is the area if $f(x)=x^{3}-18 x^{2}+87 x-70$ ?

○ If not prompted, does this answer make sense? 


\section{Question 2:}

A fire starts at midnight in a 5000 square foot house that spreads at a rate of $f(t)=e^{\frac{t}{2}}$ square feet per minute, $t$ minutes after the fire begins

- How would you calculate the amount of the house engulfed by the fire at time $a$ ?

- If a complete definite integral is prompted then ask what each aspect of the notation represents.

- The integral symbol

- bounds

- $\mathrm{f}(\mathrm{x})$

- $\mathrm{dx}$

- product between $\mathrm{f}(\mathrm{x})$ and $\mathrm{dx}$

- If an incomplete definite integral is prompted then ask what each aspect of the notation represents and prod at the mistakes.

- Can you use the definite integral structure to help determine what units of the definite integral will be?

- How much of the house is engulfed after 14 minutes?

$\circ$ Does this answer make sense, why?

- What is an answer that would not make sense?

- How much of the house is left after 15 minutes?

Does this answer make sense, why?

- What is an answer that would not make sense?

- How much of the house is left after 16 minutes?

$\circ$ Does this answer make sense, why?

- What is an answer that would not make sense?

- How long will it take for the entire house to be burned?

- Does this answer make sense, why?

- What is an answer that would not make sense?

- If the units for the x-axis is "meters" and the units for the y-axis" is "meters", what are the units for the definite integral.

○ Do any aspects of the definite integral notation support this conclusion? 


\section{APPENDIX C: Recruitment Script}

The WVU Department of Mathematics is currently conducting a research study on student understanding of first-year calculus topics, and we are looking for volunteers.

The research study will consist of one interview session per student, lasting approximately 45 minutes, where you will be videotaped working through and discussing various calculus problems.

Your participation will not affect your grade in your class.

If you are interested in furthering our knowledge of how students learn and understand calculus, please write your name and email address on the attached sheet. We will be in touch with you to set up an interview time.

Thank you so much for your time and consideration! Please let me know if you have any questions, or feel free to contact the Principal Investigator, Dr. Vicki Sealey at 304-293-5329 or sealey@math.wvu.edu. 


\section{APPENDIX D: Recruitment Email}

We are conducting a research study focused on students' ways of thinking about first year calculus concepts. We would like to interview several students that are in or have recently completed Applied Calculus and would like for you to become a participant in this study. Interviews will take approximately 45 minutes and will have flexible scheduling throughout the week. We will videotape the interviews.

If you are willing to contribute and would like to reserve an interview time and location, please reply to this email. Include your availability so we can schedule your interview promptly and at your convenience. Thank you for your consideration.

If you have any questions or concerns about this research, feel free to contact the Principal Investigator, Dr. Vicki Sealey, at 304-293-5329 or sealey@math.wvu.edu. WVU IRB approval is on file with WVU's Office or Research Integrity and Compliance (304-293-7073). 


\section{APPENDIX E: Consent Form}

\section{W. WestVirginiaUniviversity.}

\section{Definite Integral Study}

Principal Investigator

Co-Investigators

Dr. VickiSealey

Department

Cody Hood

ProtocolNumber

Study Title

Mathematics

Sponsor(ifany)

Definite Integrals in Applied Calculus

N/A

\section{Contact Persons}

If you have any questions, concerns, or complaints about this research, you should contact Dr. Vicki Sealey at (304) 293-5329. For information regarding your rights as a research subject, to discuss problems, concerns, or suggestions related to the research, to obtain information or offer input about the research, contact the Office of Research Integrity \& Compliance at (304)293-7073.

\section{Introduction}

You, have been asked to participate in this research study, which has been explained to you by . This study is being conducted by Dr. Vicki Sealey in the Department of Mathematics at West Virginia University.

\section{Purpose(s) of the Study}

Various uses of integrals in science, engineering, and mathematics and the ways in which students and experts reason about these concepts are being analyzed in this study.

\section{Description of Procedures}

This study involves videotaping of interviews with participants, where we will discuss integration. Interviews are expected to last approximately 45 minutes. Researchers will ask you a series of questions about integrals. You will be asked to think out loud and share the ways in which you think about the topics. You are welcome to use pen and paper, if you wish. Excerpts of written work may be published, and it is possible that your handwriting could be recognized.

\section{Alternatives}

You do not have to participate in this study.

\section{Benefits}

You may not receive any direct benefit from this study. The knowledge gained from this study may eventually benefit others in the teaching and learning of concepts involving integrals.

\section{Discomforts}

There are no known or expected risks from participating in this study.

\section{Financial Considerations}

There are no fees or payment for participating in this study. 